\title{
An asymmetric Suzuki-Miyaura approach to prostaglandins: Synthesis of Tafluprost
}

Roman Kučera ${ }^{\mathrm{a}}$, F. Wieland Goetzke ${ }^{\mathrm{a}}$, Stephen P. Fletcher ${ }^{\mathrm{a}}$

${ }^{a}$ Department of Chemistry, Chemistry Research Laboratory University of Oxford, 12 Mansfield Road, Oxford, OXI 3TA (UK)

\section{Supplementary Information}

\section{Table of Content}

Experimental procedures.......................................................................................................................2

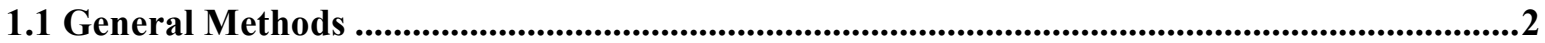

1.2 Procedures for the Synthesis of Allyl Chloride ( $( \pm)-2$..................................................................3

1.3 Procedures for the Synthesis of Boronic Acid 3 ...............................................................................9

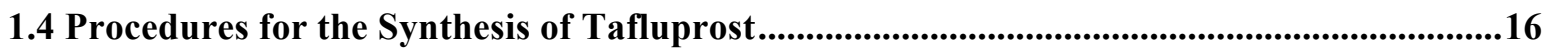

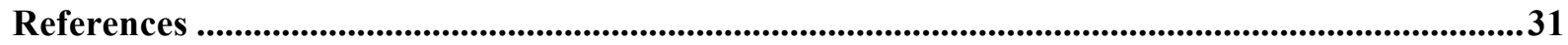

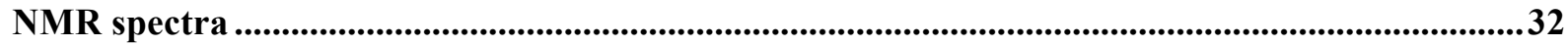




\section{Experimental procedures}

\subsection{General Methods}

Procedures using oxygen and/or moisture-sensitive materials were performed with anhydrous solvents (vide infra) under an atmosphere of anhydrous argon in flame-dried flasks, using standard Schlenk techniques. Heating was performed using DrySyn heating blocks. Analytical thin-layer chromategraphy was performed on precoated glass-backed plates (Silica Gel 60 F254; Merck), and visualised using a combination of UV light $(254 \mathrm{~nm})$ and CAM solution or aqueous basic potassium permanganate stain. Flash column chromatography was carried out using Apollo Scientific silica gel $60(0.040-0.063 \mathrm{~nm})$, Merck $60 \AA$ silica gel, VWR $(40-63 \mu \mathrm{m})$ silica gel and Sigma Aldrich silica gel.

Nuclear magnetic resonance (NMR) spectroscopy measurements were carried out at room temperature. ${ }^{1} \mathrm{H}$ NMR, ${ }^{13} \mathrm{C}$ NMR, ${ }^{19} \mathrm{~F}$ NMR, COSY, HSQC, HMBC and NOESY experiments were carried out using Bruker AVG-400 (400/100 MHz), AVH-400 (400/100 MHz), AVX-500 $(500 / 125 \mathrm{MHz})$ or AVC-500 $(500 / 125 \mathrm{MHz})$ spectrometers. Chemical shifts $(\delta)$ are reported in ppm relative to the residual solvent peak with corresponding coupling constants $(J)$ in Hertz $(\mathrm{Hz})$ and multiplicities (s: singlet, d: doublet, t: triplet, q: quartet, m: multiplet and combinations of these and app.: apparent multiplicities). Assignment follows HSQC, COSY or/and HMBC spectra, chemical shift and coupling constant analysis.

Infrared (IR, neat or thin film) spectroscopy was carried out on a Bruker Tensor 27 FT-IR spectrometer within internal calibration range of $4000-600 \mathrm{~cm}^{-1}$. The samples are reported as absorption maxima in $\mathrm{cm}^{-1}$ with corresponding relative intensities described as br (broad), s (strong), $\mathrm{m}$ (medium), and w (weak).

Chiral SFC (supercritical fluid chromatography) separations were conducted on a Waters Acquity UPC2 system using Waters Empower software. Chiralpak ${ }^{\circledR}$ columns $(150 \times 3 \mathrm{~mm}$, particle size $3 \mu \mathrm{m})$ were used as specified in the text. Solvents used were of HPLC grade (Fisher Scientific, Sigma Aldrich or Rathburn).

High Resolution Mass spectra were carried out by internal service at the University of Oxford. Electron spray ionisation $\left(\mathrm{ESI}^{+}\right)$were recorded on a Thermo Exactive with an orbitrap ion analyser.

Commercially available reagents and ligands were purchased from Sigma Aldrich, Alfa Aesar, Acros Organics, Fluorochem and Strem Chemicals and unless otherwise stated were used without further purification. $[\mathrm{Rh}(\mathrm{cod}) \mathrm{OH}]_{2}$ was synthesized according to a literature procedure ${ }^{[1]}$ Dry solvents were collected fresh from an mBraun SPS-800 solvent purification system after having passed through anhydrous alumina columns. Deuterated solvents were purchased from Sigma Aldrich. 
1.2 Procedures for the Synthesis of Allyl Chloride ( \pm )-2

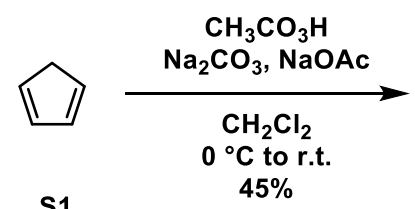

S1

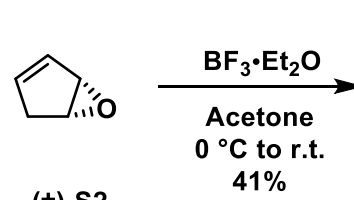

(士)-S2

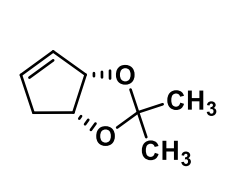

(士)-S3

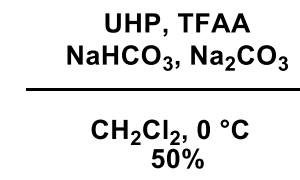

$50 \%$<smiles>CC1(C)OC2C=CC(Cl)C2O1</smiles>

(士)-2

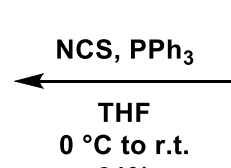

$81 \%$<smiles>CC1(C)OC2C=CC(O)C2O1</smiles>

(士)-S5

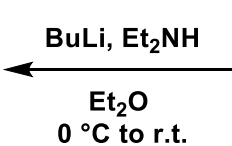

$83 \%$<smiles>CC1(C)OC2CC3OC3C2O1</smiles>

(士)-S4

Scheme S1: Overview for the synthesis of allyl chloride ( \pm )-2. 


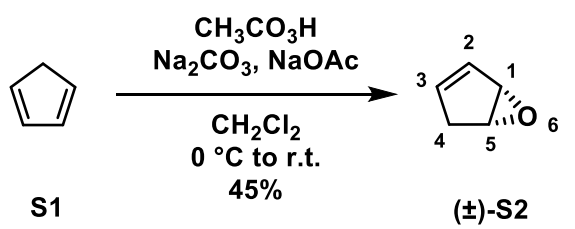

Epoxide ( \pm )-S2 was prepared according to a procedure by M. E. Jung et al. ${ }^{[2]}$

Cyclopentadiene ( $15 \mathrm{~mL}, 210 \mathrm{mmol}, 1.1$ equiv.) was added to a suspension of $\mathrm{Na}_{2} \mathrm{CO}_{3}(49.7 \mathrm{~g}, 470$ mmol, 2.47 equiv.) and $\mathrm{NaOAc}\left(950 \mathrm{mg}, 11.5 \mathrm{mmol}, 0.06\right.$ equiv.) in $\mathrm{CH}_{2} \mathrm{Cl}_{2}(235 \mathrm{~mL})$. Peracetic acid (40 wt\% in acetic acid, $32 \mathrm{~mL}, 190 \mathrm{mmol}, 1.0$ equiv.) was added dropwise at $0{ }^{\circ} \mathrm{C}$. The reaction mixture was then allowed to reach r.t. and was stirred for further $3 \mathrm{~h}$. The mixture was filtered under reduced pressure, washed with $\mathrm{CH}_{2} \mathrm{Cl}_{2}$, and concentrated under reduced pressure $\left(15^{\circ} \mathrm{C}, 250 \mathrm{mbar}\right)$. Vacuum distillation $\left(25\right.$ mbar, $\left.60{ }^{\circ} \mathrm{C}\right)$ afforded the desired product $( \pm)-\mathbf{S 2}$ as a colorless oil in a mixture with $\mathrm{CH}_{2} \mathrm{Cl}_{2}$ (estimated yield: $6.50 \mathrm{~g}, 45 \%$ ).

The spectroscopic data are in agreement with the literature. ${ }^{[2,3]}$

${ }^{1}$ H NMR $\left(400 \mathrm{MHz}, \mathrm{CDCl}_{3}\right) \delta: 6.14(\mathrm{dtd}, J=5.6,2.2,1.1 \mathrm{~Hz}, 1 \mathrm{H}, \mathrm{C}(2)-\mathrm{H}), 5.98(\mathrm{dp}, J=6.3,2.1 \mathrm{~Hz}$, $1 \mathrm{H}, \mathrm{C}(3)-\mathrm{H}), 3.91$ (td, $J=3.3,2.2 \mathrm{~Hz}, 1 \mathrm{H}, \mathrm{C}(5)-\mathrm{H}), 3.81$ (dtd, $J=2.9,1.9,1.1 \mathrm{~Hz}, 1 \mathrm{H}, \mathrm{C}(1)-\mathrm{H}), 2.63$ (dq, $\left.J=19.1,2.1 \mathrm{~Hz}, 1 \mathrm{H}, \mathrm{C}(4)-\mathrm{H}_{2}\right), 2.38$ (ddt, $J=19.1,3.5,2.1 \mathrm{~Hz}, 1 \mathrm{H}, \mathrm{C}(4)-\mathrm{H}_{2}$ ).

${ }^{13}$ C NMR (101 MHz, $\left.\mathrm{CDCl}_{3}\right)$ 8: $138.0(\mathrm{C}(3)), 131.4(\mathrm{C}(2)), 59.3(\mathrm{C}(1)), 57.0(\mathrm{C}(5)), 35.7(\mathrm{C}(4))$. 
rac-2,2-dimethyl-3a,6a-dihydro-4H-cyclopenta[d][1,3]dioxole (( \pm )-S3)

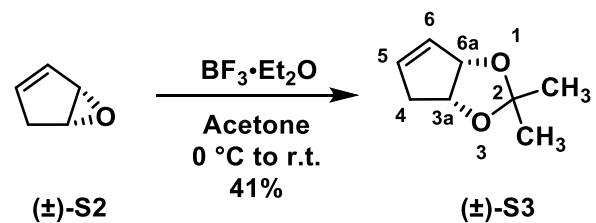

Acetal $( \pm)-\mathbf{S 3}$ was prepared according to a procedure by B. M. Trost et al. ${ }^{[4]}$

$\mathrm{BF}_{3} \cdot \mathrm{Et}_{2} \mathrm{O}(0.98 \mathrm{~mL}, 7.9 \mathrm{mmol}, 0.10$ equiv. $)$ was added dropwise to a solution of epoxide ( \pm )-S2 (6.50 $\mathrm{g}, 79.2 \mathrm{mmol}, 1.00$ equiv.) in dry acetone $(49 \mathrm{~mL})$ at $0{ }^{\circ} \mathrm{C}$. The reaction mixture was allowed to warm to r.t. and was stirred for $6 \mathrm{~h}$. The reaction mixture was then concentrated under reduced pressure (35 ${ }^{\circ} \mathrm{C}, 300$ mbar), dissolved in $\mathrm{Et}_{2} \mathrm{O}(150 \mathrm{~mL})$, washed with an aq. sat. sol. of $\mathrm{NaHCO}_{3}(2 \times 25 \mathrm{~mL})$ and an aq. sat. sol. of $\mathrm{NH}_{4} \mathrm{Cl}(2 \times 25 \mathrm{~mL})$, dried over $\mathrm{Na}_{2} \mathrm{SO}_{4}$, and concentrated under reduced pressure. Purification by gradient flash column chromatography $\left(\mathrm{Et}_{2} \mathrm{O} /\right.$ pentane $=2.5 \%$ to $\left.10 \%\right)$ afforded acetonide ( $( \pm)-\mathbf{S 3}$ as a colorless oil (4.62 g, 41\%).

The spectroscopic data are in agreement with the literature. ${ }^{[3,4]}$

${ }^{1} \mathbf{H}$ NMR $\left(400 \mathrm{MHz}, \mathrm{CDCl}_{3}\right) \delta: 5.86-5.80(\mathrm{~m}, 1 \mathrm{H}, \mathrm{C}(5)-\mathrm{H}), 5.77(\mathrm{dq}, J=5.8,1.9 \mathrm{~Hz}, 1 \mathrm{H}, \mathrm{C}(6)-\mathrm{H})$, $5.10(\mathrm{~d}, J=6.0 \mathrm{~Hz}, 1 \mathrm{H}, \mathrm{C}(6 \mathrm{a})-\mathrm{H}), 4.76$ (td, $J=5.8,1.5 \mathrm{~Hz}, 1 \mathrm{H}, \mathrm{C}(3 \mathrm{a})-\mathrm{H}), 2.64-2.45$ (m, 2H, C(4)$\left.\mathrm{H}_{2}\right), 1.41\left(\mathrm{~s}, 3 \mathrm{H}, \mathrm{CH}_{3}\right), 1.35\left(\mathrm{~s}, 3 \mathrm{H}, \mathrm{CH}_{3}\right)$.

${ }^{13}$ C NMR (101 MHz, $\left.\mathrm{CDCl}_{3}\right) \delta: 132.6(\mathrm{C}(5)), 130.8(\mathrm{C}(6)), 109.8(\mathrm{C}(2)), 85.6(\mathrm{C}(6 \mathrm{a})), 77.9(\mathrm{C}(3 \mathrm{a}))$, $38.9(\mathrm{C}(4)), 27.5\left(\mathrm{CH}_{3}\right), 25.7\left(\mathrm{CH}_{3}\right)$. 


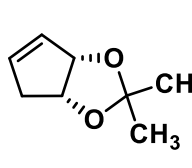

(士)-S3

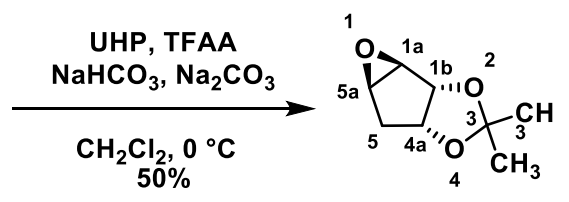

(士)-S4

Epoxide ( $( \pm)-\mathbf{S} 4$ was prepared according to a procedure by B. M. Trost et al ${ }^{[4]}$

Alkene ( \pm )-S3 (4.55 g, 32.5 mmol, 1 equiv.) and urea hydrogen peroxide (UHP) (15.3 g, $162 \mathrm{mmol}$, 5.00 equiv.) were added to a suspension of $\mathrm{NaHCO}_{3}\left(2.73 \mathrm{~g}, 32.5 \mathrm{mmol}, 1.00\right.$ equiv.) and $\mathrm{Na}_{2} \mathrm{CO}_{3}$ (10.32 g, $97.7 \mathrm{mmol}, 3.00$ equiv.) in $\mathrm{CH}_{2} \mathrm{Cl}_{2}(330 \mathrm{~mL})$ at $0{ }^{\circ} \mathrm{C}$. A solution of trifluoroacetic anhydride (TFAA) (11 mL, $81 \mathrm{mmol}, 2.5$ equiv.) in $\mathrm{CH}_{2} \mathrm{Cl}_{2}(150 \mathrm{~mL})$ was then added dropwise and the resulting mixture was stirred for $3 \mathrm{~h}$ at $0{ }^{\circ} \mathrm{C}$. The reaction mixture was then washed with an aq. sat. sol. of $\mathrm{NaHCO}_{3}(3 \times 50 \mathrm{~mL})$, an aq. sat. sol. of $\mathrm{Na}_{2} \mathrm{~S}_{2} \mathrm{O}_{3}(30 \mathrm{~mL})$, dried over $\mathrm{Na}_{2} \mathrm{SO}_{4}$, and concentrated under reduced pressure. Purification by gradient flash column chromatography $\left(\mathrm{Et}_{2} \mathrm{O} /\right.$ pentane $=10 \%$ to $\left.25 \%\right)$ afforded epoxide $( \pm)$-S4 as a colorless oil $(2.55 \mathrm{~g}, 50 \%)$.

The spectroscopic data are in agreement with the literature. ${ }^{[3,4]}$

${ }^{1} \mathbf{H}$ NMR $\left(400 \mathrm{MHz}, \mathrm{CDCl}_{3}\right) \delta: 4.55(\mathrm{~d}, J=5.5 \mathrm{~Hz}, 1 \mathrm{H}, \mathrm{C}(1 \mathrm{~b})-\mathrm{H}), 4.52(\mathrm{tdd}, J=5.6,1.9,0.9 \mathrm{~Hz}, 1 \mathrm{H}$, $\mathrm{C}(4 \mathrm{a})-\mathrm{H}), 3.60-3.54$ (m, 2H, C(1a)-H and C(5a)-H), 2.27 (dd, $\left.J=15.4,6.0 \mathrm{~Hz}, 1 \mathrm{H}, \mathrm{C}(5)-\mathrm{H}_{2}\right), 1.94$ (dt, $\left.J=15.4,2.0 \mathrm{~Hz}, 1 \mathrm{H}, \mathrm{C}(5)-\mathrm{H}_{2}\right), 1.45\left(\mathrm{~s}, 3 \mathrm{H}, \mathrm{CH}_{3}\right), 1.29\left(\mathrm{~s}, 3 \mathrm{H}, \mathrm{CH}_{3}\right)$.

${ }^{13}$ C NMR (101 MHz, $\left.\mathrm{CDCl}_{3}\right) \delta: 112.0(\mathrm{C}(3)), 81.5(\mathrm{C}(4 \mathrm{a})), 80.2(\mathrm{C}(1 \mathrm{~b})), 59.2(\mathrm{C}(1 \mathrm{a})$ or $\mathrm{C}(5 \mathrm{a})), 59.0$ (C(1a) or $\mathrm{C}(5 \mathrm{a})), 35.8(\mathrm{C}(5)), 27.4\left(\mathrm{CH}_{3}\right), 24.9\left(\mathrm{CH}_{3}\right)$. 
rac-(trans)-2,2-dimethyl-3a,6a-dihydro-4H-cyclopenta[d][1,3]dioxol-4-ol (( \pm$)-S 5)$

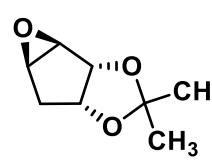

(士)-S4

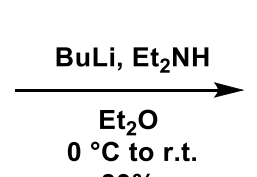

$83 \%$

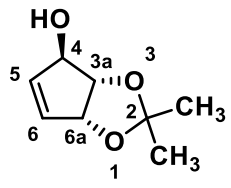

(士)-S5

Allylic alcohol ( \pm )-S5 was prepared according to a procedure by B. M. Trost et al ${ }^{[4]}$

$n$-butyl lithium (2.5 $\mathrm{M}$ in hexane, $13 \mathrm{~mL}, 32 \mathrm{mmol}, 2.0$ equiv.) was added dropwise to a solution of diethylamine (3.5 mL, $34 \mathrm{mmol}, 2.1$ equiv.) in $\mathrm{Et}_{2} \mathrm{O}(18 \mathrm{~mL})$ at $0{ }^{\circ} \mathrm{C}$. After stirring for $30 \mathrm{~min}$, a solution of epoxide $( \pm)-\mathbf{S 4}\left(2.50 \mathrm{~g}, 16.0 \mathrm{mmol}, 1.00\right.$ equiv.) in $\mathrm{Et}_{2} \mathrm{O}(18 \mathrm{~mL})$ was added dropwise and the reaction mixture was allowed to reach r.t.. After $2 \mathrm{~h}$, the reaction mixture was diluted with $\mathrm{Et}_{2} \mathrm{O}$ $(30 \mathrm{~mL})$ and washed with an aq. sat. sol. of $\mathrm{NH}_{4} \mathrm{Cl}(3 \times 15 \mathrm{~mL})$, dried over $\mathrm{MgSO}_{4}$, and concentrated under reduced pressure. Purification by gradient flash column chromatography (ethyl acetate/pentane $=10 \%$ to $50 \%)$ afforded alcohol $( \pm)$-S5 as a colorless oil $(2.08 \mathrm{~g}, 83 \%)$.

The spectroscopic data are in agreement with the literature. ${ }^{[3,4]}$

${ }^{1} \mathbf{H}$ NMR $\left(400 \mathrm{MHz}, \mathrm{CDCl}_{3}\right) \delta: 6.02(\mathrm{dt}, J=5.8,1.5 \mathrm{~Hz}, 1 \mathrm{H}, \mathrm{C}(6)-\mathrm{H}), 5.90(\mathrm{ddt}, J=5.8,2.1,0.9 \mathrm{~Hz}$, $1 \mathrm{H}, \mathrm{C}(5)-\mathrm{H}), 5.28$ (dtd, $J=5.7,1.7,0.9 \mathrm{~Hz}, 1 \mathrm{H}, \mathrm{C}(6 \mathrm{a})-\mathrm{H}), 4.78$ (d, $J=5.0 \mathrm{~Hz}, 1 \mathrm{H}, \mathrm{C}(4)-\mathrm{H}), 4.51$ (d, $J$ $=5.6 \mathrm{~Hz}, 1 \mathrm{H}, \mathrm{C}(3 \mathrm{a})-\mathrm{H}), 2.20(\mathrm{~m}, 1 \mathrm{H}, \mathrm{OH}), 1.39\left(\mathrm{~s}, 3 \mathrm{H}, \mathrm{CH}_{3}\right), 1.34\left(\mathrm{~s}, 3 \mathrm{H}, \mathrm{CH}_{3}\right)$.

${ }^{13}$ C NMR (101 MHz, $\left.\mathrm{CDCl}_{3}\right)$ 8: $135.7(\mathrm{C}(6)), 134.8$ (C(5)), 111.9 (C(2)), 86.1 (C(3a)), 84.4 (C(6a)), $81.1(\mathrm{C}(4)), 27.4\left(\mathrm{CH}_{3}\right), 25.8\left(\mathrm{CH}_{3}\right)$. 
<smiles>CC1(C)O[C@@H]2C=C[C@@H](O)[C@H]2O1</smiles>

(士)-S5

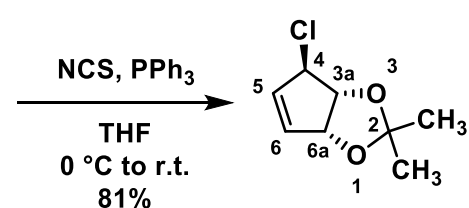

(士)-2

Allylic alcohol $( \pm)-2$ was prepared according to a procedure by F. W. Goetzke et al ${ }^{[3]}$

Triphenylphosphine (5.04 g, $19.2 \mathrm{mmol}, 1.50$ equiv.) and $N$-chlorosuccinimide (NCS) $(2.74 \mathrm{~g}$, 20.5 mmol, 1.60 equiv.) were added to a solution of alcohol $( \pm)$-S5 (2.00 g, $12.8 \mathrm{mmol}, 1.00$ equiv.) in THF $(20 \mathrm{~mL})$ at $0{ }^{\circ} \mathrm{C}$. After $1 \mathrm{~h}$, the reaction mixture was allowed to reach r.t. and was stirred for additional $1 \mathrm{~h}$. The reaction mixture was then filtered through a pad of Celite and concentrated under reduced pressure. Purification by gradient flash column chromatography $\left(\mathrm{CH}_{2} \mathrm{Cl}_{2} /\right.$ pentane $=0 \%$ to $50 \%$ ) afforded allyl chloride $( \pm)-2$ as a colorless oil ( $1.81 \mathrm{~g}, 81 \%)$.

The spectroscopic data are in agreement with the literature. ${ }^{[3]}$

${ }^{1} \mathbf{H}$ NMR $\left(400 \mathrm{MHz}, \mathrm{CDCl}_{3}\right) \delta: 6.01$ (ddt, $\left.J=5.7,1.7,0.7 \mathrm{~Hz}, 1 \mathrm{H}, \mathrm{C}(6)-\mathrm{H}\right), 5.91$ (ddt, $J=5.6,2.3,0.9$ $\mathrm{Hz}, 1 \mathrm{H}, \mathrm{C}(5)-\mathrm{H}), 5.32$ (dq, $J=5.6,1.4 \mathrm{~Hz}, 1 \mathrm{H}, \mathrm{C}(6 \mathrm{a})-\mathrm{H}), 4.81$ (ddt, $J=2.4,1.5,0.7 \mathrm{~Hz}, 1 \mathrm{H}, \mathrm{C}(4)-\mathrm{H})$, 4.79 (dd, $J=5.5,0.8 \mathrm{~Hz}, 1 \mathrm{H}, \mathrm{C}(3 \mathrm{a})-\mathrm{H}), 1.38\left(\mathrm{~s}, 3 \mathrm{H}, \mathrm{CH}_{3}\right), 1.35$ (s, 3H, $\left.\mathrm{CH}_{3}\right)$.

${ }^{13}$ C NMR (101 MHz, $\left.\mathrm{CDCl}_{3}\right)$ ): 135.7 (C(6)), $133.6(\mathrm{C}(5)), 112.3$ (C(2)), 86.1 (C(3a)), 84.4 (C(6a)), $65.1(\mathrm{C}(4)), 27.6\left(\mathrm{CH}_{3}\right), 26.3\left(\mathrm{CH}_{3}\right)$. 
1.3 Procedures for the Synthesis of Boronic Acid 3

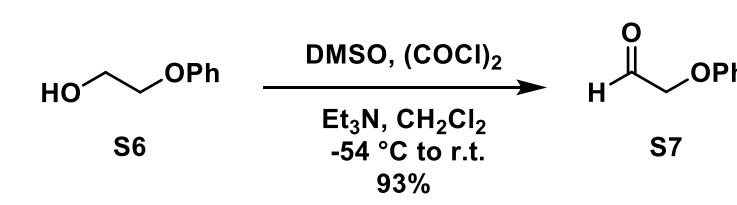

$(\mathrm{HO})_{2} \mathrm{P}^{\mathrm{O}} \mathrm{PPh}^{\mathrm{F}}$

3

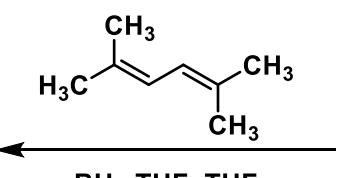

$\mathrm{BH}_{3} \cdot$ THF, THF $0^{\circ} \mathrm{C}$ to r.t.

then aq. $\mathrm{H}_{2} \mathrm{CO}$
1) $\mathrm{TMSC} \equiv \mathrm{CH}, \mathrm{BuLi}, \mathrm{THF}$ $-78^{\circ} \mathrm{C}$ to r.t.

2) TBAF, THF, r.t.

$80 \%$ (2 steps)

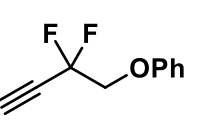

$\mathbf{S 1 0}$ h

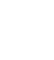

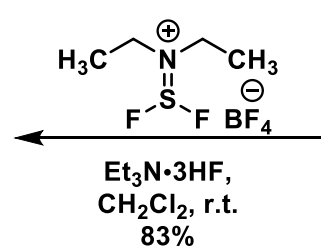<smiles>C#CC(=O)COc1ccccc1</smiles>

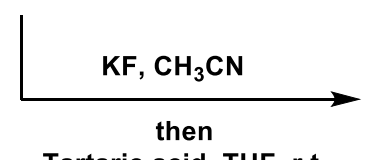

Tartaric acid, THF, r.t. $80 \%$ (2 steps)

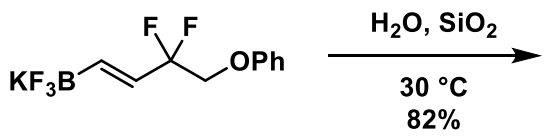

S11

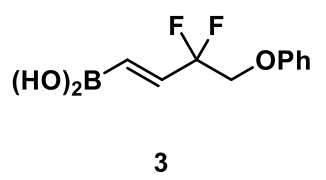

Scheme S2: Overview for the synthesis of boronic acid 3. 


\section{2-phenoxyacetaldehyde (S7)}

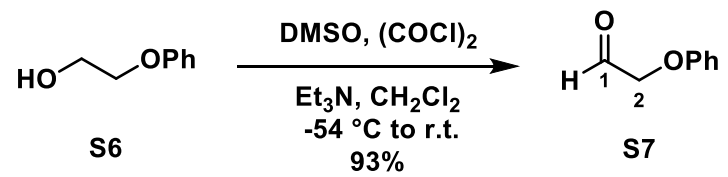

Aldehyde $\mathbf{S} 7$ was prepared according to a procedure by D. R. Dragoli et al ${ }^{[5]}$

A solution of DMSO (11 mL, $160 \mathrm{mmol}, 2.2$ equiv.) in $\mathrm{CH}_{2} \mathrm{Cl}_{2}(36 \mathrm{~mL})$ was added dropwise over 30 min to a solution of oxalyl chloride $(6.7 \mathrm{ml}, 80 \mathrm{mmol}, 1.1$ equiv. $)$ in $\mathrm{CH}_{2} \mathrm{Cl}_{2}(290 \mathrm{~mL})$ at $-54{ }^{\circ} \mathrm{C}$. After $10 \mathrm{~min}$, a solution of 2-phenoxyethanol (10.0 g, $72.4 \mathrm{mmol}, 1.00$ equiv.) in $\mathrm{CH}_{2} \mathrm{Cl}_{2}$ (70 mL) was added dropwise over $30 \mathrm{~min}$ and the reaction mixture was stirred with mechanical stirrer for additional $60 \mathrm{~min}$. Triethylamine ( $40 \mathrm{~mL}, 290 \mathrm{mmol}, 4.0$ equiv.) was then added dropwise over $20 \mathrm{~min}$. After 60 $\mathrm{min}$, water $(320 \mathrm{~mL})$ was added and the reaction mixture was allowed to reach r.t.. The organic phase was separated and the aq. phase extracted with $\mathrm{CH}_{2} \mathrm{Cl}_{2}(4 \times 50 \mathrm{~mL})$. The combined organic phases were washed with $1 \mathrm{M}$ aq. $\mathrm{HCl}(5 \times 50 \mathrm{~mL})$, brine $(2 \times 60 \mathrm{~mL})$, an aq. sat. sol. of $\mathrm{NaHCO}_{3}(3 \times 50$ $\mathrm{mL}$ ), dried over $\mathrm{MgSO}_{4}$, and concentrated under reduced pressure. The crude aldehyde $\mathbf{S} 7$ was obtained as a pale yellow oil $(9.17 \mathrm{~g}, 93 \%)$ and used in the next step without further purification.

The spectroscopic data are in agreement with the literature. ${ }^{[6]}$

${ }^{1}$ H NMR (400 MHz, $\left.\mathrm{CDCl}_{3}\right) \delta: 9.87(\mathrm{t}, J=1.1 \mathrm{~Hz}, 1 \mathrm{H}, \mathrm{C}(1)-\mathrm{H}), 7.36-7.26\left(\mathrm{~m}, 2 \mathrm{H}, \mathrm{C}_{\text {meta }}-\mathrm{H}\right), 7.06$ $7.00\left(\mathrm{~m}, 1 \mathrm{H}, \mathrm{C}_{\mathrm{para}}-\mathrm{H}\right), 6.94-6.87\left(\mathrm{~m}, 2 \mathrm{H}, \mathrm{C}_{\text {ortho }}-\mathrm{H}\right), 4.57\left(\mathrm{~d}, J=1.1 \mathrm{~Hz}, 2 \mathrm{H}, \mathrm{C}(2)-\mathrm{H}_{2}\right)$.

${ }^{13}$ C NMR (101 MHz, $\left.\mathrm{CDCl}_{3}\right)$ \&: $199.6(\mathrm{C}(1)), 157.8\left(\mathrm{C}_{\mathrm{q}}\right), 129.9\left(\mathrm{C}_{\text {meta }}\right), 122.1\left(\mathrm{C}_{\text {para }}\right), 114.7\left(\mathrm{C}_{\text {ortho }}\right)$, $72.8(\mathrm{C}(2))$. 
rac-1-phenoxybut-3-yn-2-ol (( \pm )-S8)

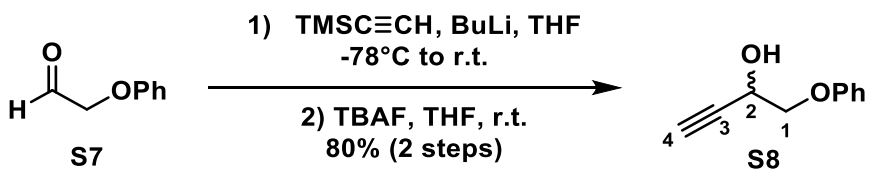

Alcohol ( \pm )-S8 was prepared according to a procedure by E. J. Corey et al. ${ }^{[7]}$

$n$-butyl lithium $(2.5 \mathrm{M}$ in hexane, $22 \mathrm{~mL}, 55 \mathrm{mmol}, 1.5$ equiv.) was added to a solution of ethynyltrimethylsilane $\left(10 \mathrm{~mL}, 73 \mathrm{mmol}, 2.0\right.$ equiv.) in THF $(220 \mathrm{~mL})$ at $-78{ }^{\circ} \mathrm{C}$. After stirring for 30 min, a solution of aldehyde $\mathbf{S} 7(5.00 \mathrm{~g}, 36.7 \mathrm{mmol}, 1.00$ equiv.) in THF (30 mL) was added dropwise over $15 \mathrm{~min}$. After $30 \mathrm{~min}$, the reaction mixture was allowed reach r.t. and stirred at this temperature for $30 \mathrm{~min}$. The reaction mixture was then quenched with an aq. sat. sol. of $\mathrm{NH}_{4} \mathrm{Cl}(150 \mathrm{~mL})$ and extracted with $\mathrm{Et}_{2} \mathrm{O}(3 \times 100 \mathrm{~mL})$. The combined organic phases were washed with brine $(100 \mathrm{~mL})$, dried over $\mathrm{Na}_{2} \mathrm{SO}_{4}$, and concentrated under reduced pressure.

The residue was dissolved in THF $(150 \mathrm{~mL})$ and a solution of tetrabutylammonium fluoride (TBAF) ( $1 \mathrm{M}$ in THF, $38 \mathrm{ml}, 37 \mathrm{mmol}, 1.0$ equiv.) was added dropwise at $0{ }^{\circ} \mathrm{C}$. The resulting orange solution was stirred for $20 \mathrm{~min}$ at r.t. and then poured onto an aq. sat. sol. of $\mathrm{NaHCO}_{3}$. The aq. phase was extracted with $\mathrm{Et}_{2} \mathrm{O}(3 \times 100 \mathrm{~mL})$, the combined organic phases were washed with brine $(100 \mathrm{~mL})$, dried over $\mathrm{Na}_{2} \mathrm{SO}_{4}$ and concentrated under reduced pressure. Purification by gradient flash column chromatography (ethyl acetate/hexane $=5 \%$ to $35 \%$ ) afforded alcohol $( \pm$ )-S8 as a colorless solid (4.75 $\mathrm{g}, 80 \%$, over 2 steps).

The spectroscopic data are in agreement with the literature. ${ }^{[7]}$

${ }^{1} \mathbf{H}$ NMR $\left(400 \mathrm{MHz}, \mathrm{CDCl}_{3}\right) \delta: 7.35-7.26\left(\mathrm{~m}, 2 \mathrm{H}, \mathrm{C}_{\text {meta }}-\mathrm{H}\right), 7.00\left(\mathrm{tt}, J=7.3,1.1 \mathrm{~Hz}, 1 \mathrm{H}, \mathrm{C}_{\mathrm{para}}-\mathrm{H}\right)$, $6.97-6.90\left(\mathrm{~m}, 2 \mathrm{H}, \mathrm{C}_{\text {ortho }}-\mathrm{H}\right), 4.77$ (dddd, $\left.J=7.4,5.7,3.6,2.2 \mathrm{~Hz}, 1 \mathrm{H}, \mathrm{C}(1)-\mathrm{H}\right), 4.16$ (dd, $J=9.6,3.7$ $\left.\mathrm{Hz}, 1 \mathrm{H}, \mathrm{C}(1)-\mathrm{H}_{2}\right), 4.09\left(\mathrm{dd}, J=9.6,7.0 \mathrm{~Hz}, 1 \mathrm{H}, \mathrm{C}(1)-\mathrm{H}_{2}\right), 2.71(\mathrm{~d}, J=5.4 \mathrm{~Hz}, 1 \mathrm{H}, \mathrm{OH}), 2.54(\mathrm{~d}, J=$ $2.3 \mathrm{~Hz}, 1 \mathrm{H}, \mathrm{C}(4)-\mathrm{H})$.

${ }^{13}$ C NMR (101 MHz, $\left.\mathrm{CDCl}_{3}\right)$ \&: $158.2\left(\mathrm{C}_{\mathrm{q}}\right), 129.7\left(\mathrm{C}_{\text {meta }}\right), 121.7\left(\mathrm{C}_{\text {para }}\right), 114.9\left(\mathrm{C}_{\text {ortho }}\right), 81.2(\mathrm{C}(4)), 74.5$ $(\mathrm{C}(5)), 71.4(\mathrm{C}(2)), 61.4(\mathrm{C}(1))$. 
<smiles>C#C[C@H](O)COc1ccccc1</smiles>

S8

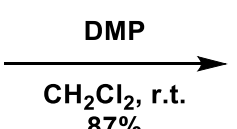

$87 \%$<smiles>C#CC(=O)COc1ccccc1</smiles>

S9

Dess-Martin periodinane (DMP) (21.9 g, $51.6 \mathrm{mmol}, 1.20$ equiv.) was added to a solution of alcohol ( \pm )-S8 (6.97 g, $43.0 \mathrm{mmol}, 1.00$ equiv.) in $\mathrm{CH}_{2} \mathrm{Cl}_{2}(200 \mathrm{~mL})$ and the resulting solution was stirred for $50 \mathrm{~min}$ at r.t. The reaction mixture was then poured onto an aq. sat. sol. of $\mathrm{NaHCO}_{3}(200 \mathrm{~mL})$ and the aq. phase was extracted with $\mathrm{CH}_{2} \mathrm{Cl}_{2}(3 \times 100 \mathrm{~mL})$. The combined organic phases were washed with brine $(100 \mathrm{~mL})$, dried over $\mathrm{Na}_{2} \mathrm{SO}_{4}$, and concentrated under reduced pressure. The colorless residue was passed through a short plug of silica $(6 \mathrm{~cm})$ and eluted with $15 \%$ mixture of ethyl acetate in hexane. The purified product $\mathbf{S 9}$ was obtained as a colourless crystalline solid $(5.99 \mathrm{~g}, 87 \%,>98 \%$ purity) contaminated with orange residue (product of decomposition on silica).

The spectroscopic data are in agreement with the literature. ${ }^{[8]}$

${ }^{1} \mathbf{H}$ NMR $\left(400 \mathrm{MHz}, \mathrm{CDCl}_{3}\right) \delta: 7.34-7.27\left(\mathrm{~m}, 2 \mathrm{H}, \mathrm{C}_{\text {meta }}-\mathrm{H}\right), 7.05-6.99\left(\mathrm{~m}, 1 \mathrm{H}, \mathrm{C}_{\mathrm{para}}-\mathrm{H}\right), 6.93-6.87$ (m, 2H, $\left.\mathrm{C}_{\text {ortho }}-\mathrm{H}\right), 4.75$ (s, 2H, C(1)- $\left.\mathrm{H}_{2}\right), 3.39$ (s, 1H, C(4)-H).

${ }^{13}$ C NMR (101 MHz, $\left.\mathrm{CDCl}_{3}\right) \delta: 182.7(\mathrm{C}(2)), 157.6\left(\mathrm{C}_{\mathrm{q}}\right), 129.7\left(\mathrm{C}_{\text {meta }}\right), 122.1\left(\mathrm{C}_{\text {para }}\right), 114.8\left(\mathrm{C}_{\text {ortho }}\right)$, $82.4(\mathrm{C}(4)), 79.1(\mathrm{C}(3)), 73.5(\mathrm{C}(1))$. 
((2,2-difluorobut-3-yn-1-yl)oxy)benzene (S10)

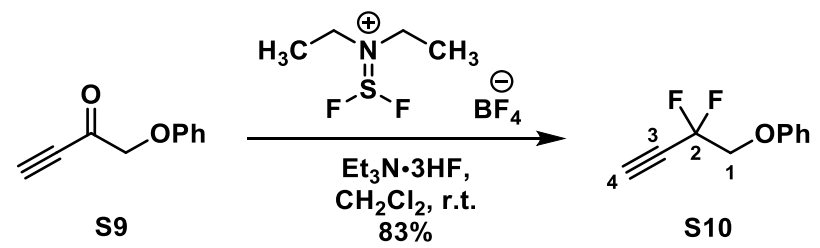

Compound S10 was prepared according to a procedure by J.-F. Syu et al ${ }^{[9]}$

Triethylamine trihydrofluoride ( $12 \mathrm{~mL}, 74 \mathrm{mmol}, 2.0$ equiv.) was added to a suspension of XtalFluorE $\left(12.7 \mathrm{~g}, 55.3 \mathrm{mmol}, 1.50\right.$ equiv.) in $\mathrm{CH}_{2} \mathrm{Cl}_{2}(13 \mathrm{~mL})$ at r.t. After complete dissolution of solid reagent, a solution of ketone $\mathbf{S 9}\left(5.90 \mathrm{~g}, 36.8 \mathrm{mmol}, 1.00\right.$ equiv.) in $\mathrm{CH}_{2} \mathrm{Cl}_{2}(30 \mathrm{~mL})$ was added and the resulting mixture was stirred for $18 \mathrm{~h}$. The reaction mixture was then poured onto an aq. sat. sol. of $\mathrm{NaHCO}_{3}(150 \mathrm{~mL})$ and was extracted with $\mathrm{Et}_{2} \mathrm{O}(3 \times 100 \mathrm{~mL})$. The combined organic phases were washed with brine $(100 \mathrm{~mL})$, dried over $\mathrm{Na}_{2} \mathrm{SO}_{4}$, and concentrated under reduced pressure $\left(30{ }^{\circ} \mathrm{C}, 50\right.$ mbar). Purification by gradient flash column chromatography (ethyl acetate/pentane $=2 \%$ to $6 \%$ ) afforded alkyne $\mathbf{S 1 0}$ as a pale yellow oil (5.57 g, 83\%).

The spectroscopic data are in agreement with the literature. ${ }^{[9]}$

${ }^{1} \mathbf{H}$ NMR $\left(400 \mathrm{MHz}, \mathrm{CDCl}_{3}\right) \delta: 7.37-7.28\left(\mathrm{~m}, 2 \mathrm{H}, \mathrm{C}_{\text {meta }}-\mathrm{H}\right), 7.07-7.01\left(\mathrm{~m}, 1 \mathrm{H}, \mathrm{C}_{\text {para }}-\mathrm{H}\right), 6.99-6.94$ (m, 2H, $\left.\mathrm{C}_{\text {ortho }}-\mathrm{H}\right), 4.30$ (t, $\left.J=11.3 \mathrm{~Hz}, 2 \mathrm{H}, \mathrm{C}(1)-\mathrm{H}_{2}\right), 2.86$ (t, $\left.J=5.1 \mathrm{~Hz}, 1 \mathrm{H}, \mathrm{C}(4)-\mathrm{H}\right)$.

${ }^{13} \mathbf{C}$ NMR (126 MHz, $\left.\mathrm{CDCl}_{3}\right) \delta: 158.0\left(\mathrm{C}_{\mathrm{q}}\right), 129.8\left(\mathrm{C}_{\text {meta }}\right), 122.3\left(\mathrm{C}_{\text {para }}\right), 115.2\left(\mathrm{C}_{\text {ortho }}\right), 111.0(\mathrm{C}(2))$, $77.2(\mathrm{C}(4)), 74.8(\mathrm{C}(3)), 70.3(\mathrm{C}(1))$. (Peak reported at $77.1 \mathrm{ppm}$ overlaps with solvent peak).

${ }^{19}$ F NMR (377 MHz, $\mathrm{CDCl}_{3},{ }^{1} \mathrm{H}$ decoupled) $\delta:-93.34$. 
Potassium (E)-(3,3-Difluoro-4-phenoxybut-1-en-1-yl)- trifluoroborate (S11)

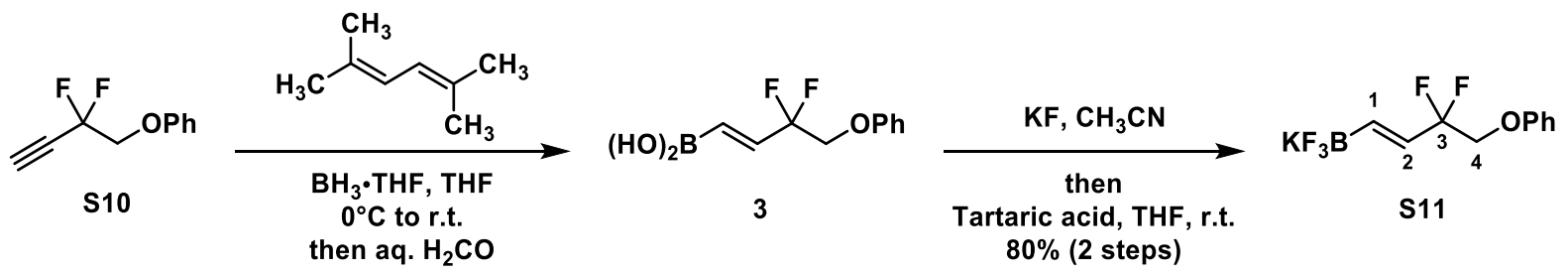

Compound S11 was prepared according to a procedure by J.-F. Syu et al. ${ }^{[9]}$

A solution of borane tetrahydrofuran complex ( $1 \mathrm{M}$ in THF, $61 \mathrm{~mL}, 61 \mathrm{mmol}, 2.5$ equiv.) was added to a solution of 2,5-dimethylhexa-2,4-diene $\left(20 \mathrm{~mL}, 130 \mathrm{mmol}, 5.5\right.$ equiv.) in THF $(11 \mathrm{~mL})$ at $0{ }^{\circ} \mathrm{C}$. After stirring for $3 \mathrm{~h}$, a solution of alkyne $\mathbf{S 1 0}$ (4.44 g, $24.4 \mathrm{mmol}, 1.00$ equiv.) in THF (24 mL) was added dropwise and the reaction mixture was stirred overnight at $r$.t. The reaction mixture was then carefully quenched by addition of water $\left(9.3 \mathrm{~mL}\right.$ ) (evolution of gas observed) at $0{ }^{\circ} \mathrm{C}$. After stirring for $90 \mathrm{~min}$ at r.t., $37 \%$ aq. sol. of formaldehyde $(22 \mathrm{~mL})$ was added (slightly exothermic reaction, exothermicity compensate by water bath) and the resulting mixture was stirred overnight at r.t.

The reaction mixture was then poured onto brine $(40 \mathrm{~mL})$, and the aq. phase was extracted with ethyl acetate $(4 \times 60 \mathrm{~mL})$. The combined organic phases were dried over $\mathrm{Na}_{2} \mathrm{SO}_{4}$ and concentrated under reduced pressure to provide crude boronic acid 3 together with $(E)$-2,2,5-trimethylhex-3-en-1-ol as a pale yellow oil (25.4 g).

A solution of potassium fluoride $(5.14 \mathrm{~g}, 88.5 \mathrm{mmol}, 3.63$ equiv.) in water $(7.6 \mathrm{~mL})$ was added to a solution of above prepared boronic acid 3 in $\mathrm{CH}_{3} \mathrm{CN}(100 \mathrm{~mL})$. The resulting mixture was vigorously stirred until dissolution was achieved $(1 \mathrm{~h})$. A solution of L-(+)-tartaric acid $(6.84 \mathrm{~g}, 45.6 \mathrm{mmol}, 1.87$ equiv.) in THF ( $35 \mathrm{~mL}$ ) was then added dropwise over $20 \mathrm{~min}$ (formation of a white precipitate). After stirring for $30 \mathrm{~min}$, the solids were filtered off and washed with $\mathrm{CH}_{3} \mathrm{CN}(50 \mathrm{~mL})$. The collected solution was concentrated under reduced pressure and the obtained residue was suspended in $\mathrm{Et}_{2} \mathrm{O}(20$ $\mathrm{mL}$ ). After stirring for $1 \mathrm{~h}$, the solid product was filtered off and washed with hexane to provide trifluoroborate $\mathbf{S 1 1}$ as a pale yellow solid (4.83 g, 68\%).

Note: The trifluoroborate $\mathbf{S 1 1}$ is partially soluble in (E)-2,2,5-trimethylhex-3-en-1-ol formed as a byproduct in the hydroboration step. Therefore, an additional portion of trifluoroborate can be isolated from the solution obtained during the last filtration by removal of volatiles on rotavap and evaporation of the side product on kugelrohr, followed by precipitation of compound $\mathbf{S 1 1}$ with hexane $(0.85 \mathrm{~g}$, $12 \%$; combined yield: $80 \%$ ).

The spectroscopic data are in agreement with the literature. ${ }^{[9]}$

${ }^{1} \mathrm{H}$ NMR $\left(500 \mathrm{MHz},\left(\mathrm{CD}_{3}\right)_{2} \mathrm{SO}\right) \delta: 7.37-7.20\left(\mathrm{~m}, 2 \mathrm{H}, \mathrm{C}_{\text {meta }}-\mathrm{H}\right), 7.07-6.88\left(\mathrm{~m}, 3 \mathrm{H}, \mathrm{C}_{\text {ortho }}-\mathrm{H}\right.$ and $\left.\mathrm{C}_{\text {para }}-\mathrm{H}\right), 6.08(\mathrm{dq}, J=18.3,3.1 \mathrm{~Hz}, 1 \mathrm{H}, \mathrm{C}(1)-\mathrm{H}), 5.74(\mathrm{dt}, J=18.2,11.4 \mathrm{~Hz}, 1 \mathrm{H}, \mathrm{C}(2)-\mathrm{H}), 4.24(\mathrm{t}, J=$ $\left.13.2 \mathrm{~Hz}, 2 \mathrm{H}, \mathrm{C}(4)-\mathrm{H}_{2}\right)$.

${ }^{13} \mathrm{C}$ NMR $\left(126 \mathrm{MHz},\left(\mathrm{CD}_{3}\right)_{2} \mathrm{SO}\right) \delta: 157.9\left(\mathrm{C}_{\mathrm{q}}\right), 129.6\left(\mathrm{C}_{\text {meta }}\right), 126.1$ (tq, $\left.J=24.6,3.9 \mathrm{~Hz}, \mathrm{C}(2)\right), 121.2$ $\left(\mathrm{C}_{\text {para }}\right), 119.7$ ( $\left.\mathrm{t}, J=238.6 \mathrm{~Hz}, \mathrm{C}(3)\right), 114.7$ ( $\left.\mathrm{C}_{\text {ortho }}\right), 68.6$ (t, $\left.J=31.3 \mathrm{~Hz}, \mathrm{C}(4)\right)$ ). (The $\mathrm{C}-\mathrm{B}$ signal was not observed due to quadrupolar relaxation.)

${ }^{19}$ F NMR (471 MHz, $\left(\mathrm{CD}_{3}\right)_{2} \mathrm{SO},{ }^{1} \mathrm{H}$ decoupled) $\delta:-101.32,-139.42$. 
(E)-(3,3-difluoro-4-phenoxybut-1-en-1-yl)boronic acid (3)

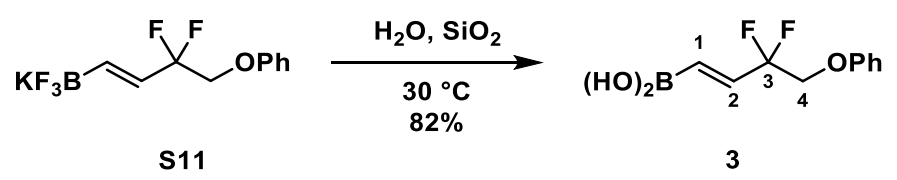

Compound $\mathbf{3}$ was prepared according to a procedure by J.-F. Syu et al. ${ }^{[9]}$

A suspension of trifluorobotonate $\mathbf{S 1 1}$ (4.75 g, $16.4 \mathrm{mmol}, 1.00$ equiv.) and silica gel (984 mg, 16.4 mmol, 1.00 equiv.) in water $(16 \mathrm{~mL})$ were stirred at $30{ }^{\circ} \mathrm{C}$ overnight. The solids were collected by vacuum filtration (pore size $\mathrm{S} 4$ ) and washed with water $(5 \mathrm{~mL})$. The solid was extracted several times with $\mathrm{CH}_{3} \mathrm{CN}$. The collected solution was concentrated under reduced pressure and the obtained pale yellow product was triturated with pentane $(3 \times 5 \mathrm{~mL})$ to afford boronic acid $\mathbf{3}$ as a white solid $(3.05 \mathrm{~g}$, $82 \%)$.

The spectroscopic data are in agreement with the literature. ${ }^{[9]}$

${ }^{1}$ H NMR $\left(500 \mathrm{MHz},\left(\mathrm{CD}_{3}\right)_{2} \mathrm{SO}\right) \delta: 8.12(\mathrm{~s}, 2 \mathrm{H}, \mathrm{OH}), 7.37-7.26\left(\mathrm{~m}, 2 \mathrm{H}, \mathrm{C}_{\text {meta }}-\mathrm{H}\right), 7.05-6.94(\mathrm{~m}, 3 \mathrm{H}$, $\mathrm{C}_{\text {ortho }}-\mathrm{H}$ and $\left.\mathrm{C}_{\text {para }}-\mathrm{H}\right), 6.56(\mathrm{dt}, J=18.3,11.1 \mathrm{~Hz}, 1 \mathrm{H}, \mathrm{C}(1)-\mathrm{H}), 6.07(\mathrm{dt}, J=18.3,2.4 \mathrm{~Hz}, 1 \mathrm{H}, \mathrm{C}(2)-\mathrm{H})$, $4.37\left(\mathrm{t}, J=12.9 \mathrm{~Hz}, 2 \mathrm{H}, \mathrm{C}(4)-\mathrm{H}_{2}\right)$.

${ }^{13} \mathrm{C}$ NMR (126 MHz, $\left(\mathrm{CD}_{3}\right)_{2} \mathrm{SO},{ }^{19} \mathrm{~F}$ decoupled) $\delta: 157.6\left(\mathrm{C}_{\mathrm{q}}\right), 138.2(\mathrm{C}(2)), 129.6\left(\mathrm{C}_{\text {meta }}\right), 121.5$ $\left(\mathrm{C}_{\text {para }}\right), 118.8(\mathrm{C}(3)), 114.8\left(\mathrm{C}_{\text {ortho }}\right), 68.2(\mathrm{C}(4))$. (The $\mathrm{C}-\mathrm{B}$ signal was not observed due to quadrupolar relaxation.)

${ }^{19}$ F NMR (471 MHz, $\left(\mathrm{CD}_{3}\right)_{2} \mathrm{SO},{ }^{1} \mathrm{H}$ decoupled) $\delta:-103.99$. 


\subsection{Procedures for the Synthesis of Tafluprost}
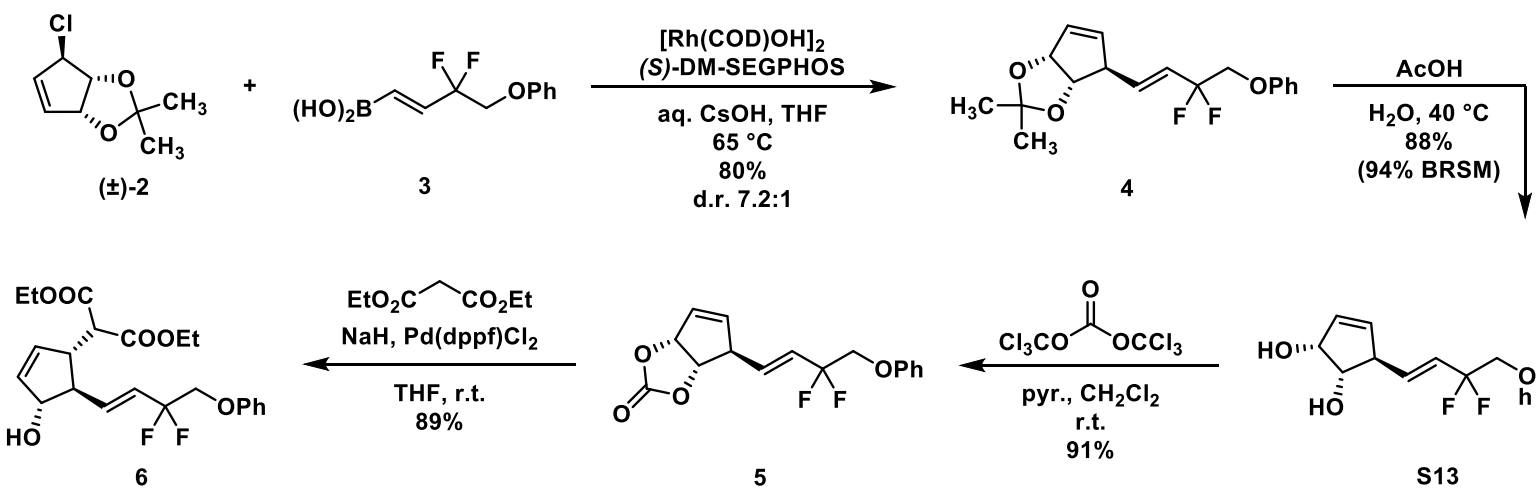
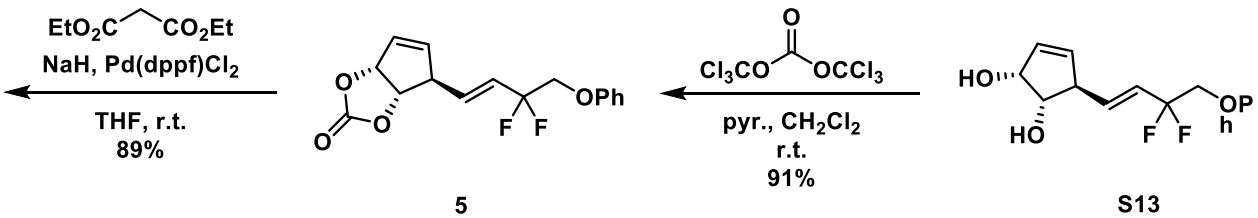

S13

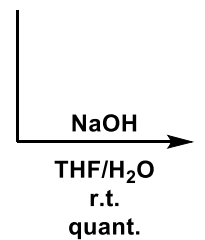<smiles>O=C(O)C(C(=O)O)[C@H]1C=C[C@H](O)[C@@H]1/C=C/C(F)(F)COc1ccccc1</smiles>

S15

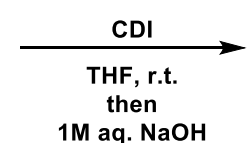<smiles>O=C(O)C[C@H]1C=C[C@@H](O)[C@@H]1/C=C/C(F)(F)COc1ccccc1</smiles>

7
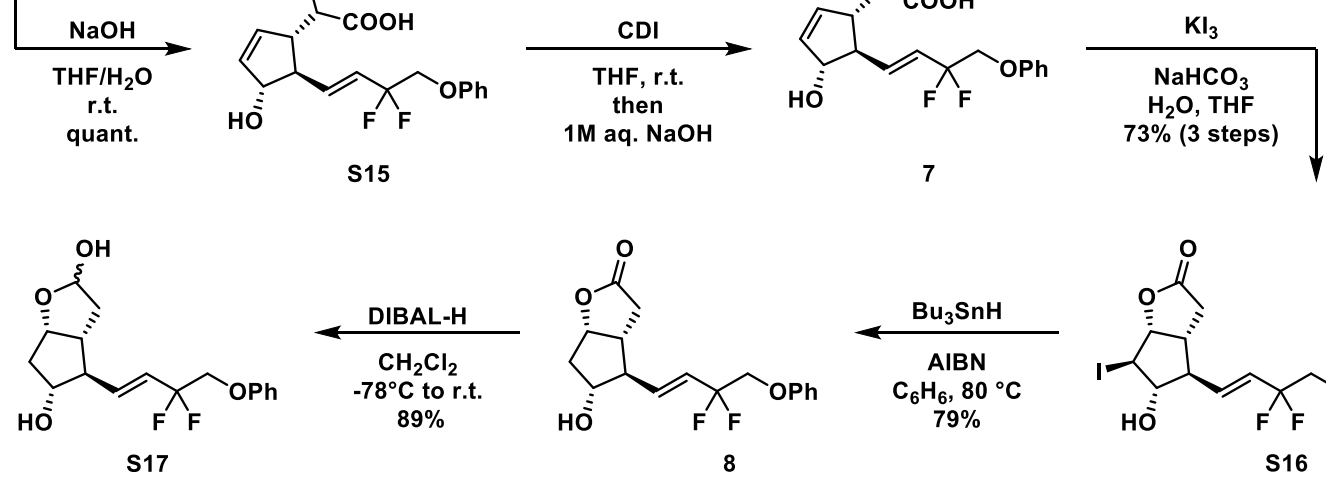

$89 \%$
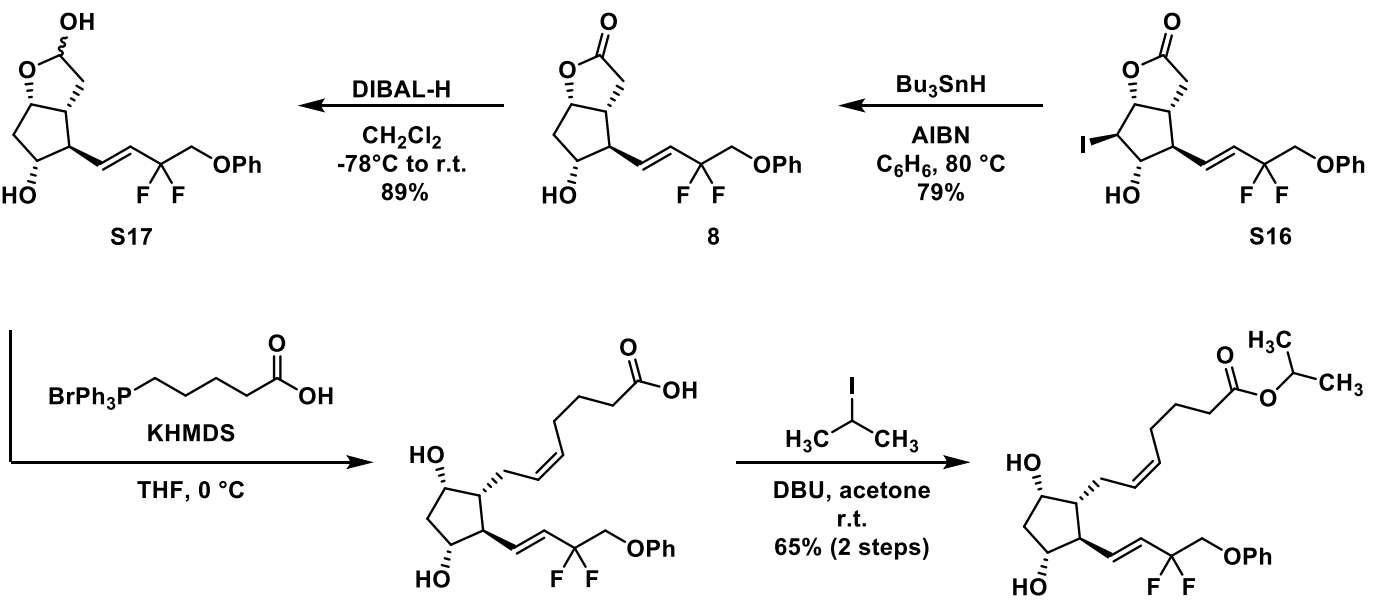

S18

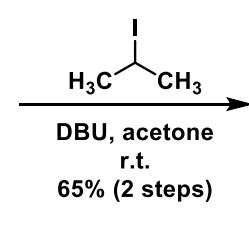

r.t.
$65 \%$ (2 steps)

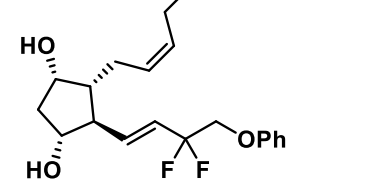

Tafluprost (1)

Scheme S3: Overview for the synthesis of Tafluprost (1). 
(3aS,4S,6aR)-4-((E)-3,3-difluoro-4-phenoxybut-1-en-1-yl)-2,2-dimethyl-3a,6a-dihydro-4Hcyclopenta[d][1,3]dioxole (4)<smiles>CC1(C)O[C@H]2C=CC(Cl)[C@H]2O1</smiles>

$( \pm)-2$

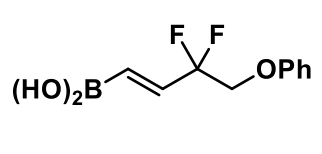

3

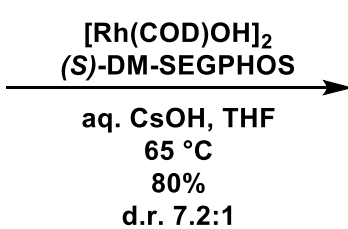

d.r. 7.2:1

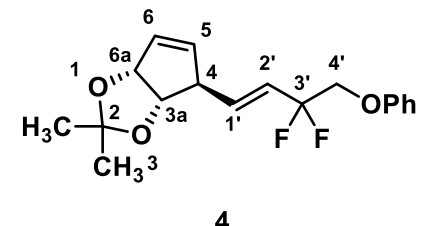

4

Compound 4 was prepared adopting a procedure by F. W. Goetzke et al. ${ }^{[3]}$

An aq. sol. of $\mathrm{CsOH}(50 \mathrm{wt} \%, 330 \mu \mathrm{L}, 1.9 \mathrm{mmol}, 1.0$ equiv.) was added to a solution of (S)-DMSEGPHOS (82.4 mg, $114 \mu \mathrm{mol}, 6.0 \mathrm{~mol} \%)$ and $[\mathrm{Rh}(\operatorname{cod}) \mathrm{OH}]_{2}(21.7 \mathrm{mg}, 47.5 \mu \mathrm{mol}, 2.5 \mathrm{~mol} \%)$ in THF $(3.3 \mathrm{~mL})$ and the resulting mixture was stirred at $65{ }^{\circ} \mathrm{C}$ for $30 \mathrm{~min}$. Then, a solution of boronic acid 3 (650 mg, $2.85 \mathrm{mmol}, 1.50$ equiv.) and allyl chloride ( \pm )-2 (300 $\mu \mathrm{L}, 1.9 \mathrm{mmol}, 1.0$ equiv.) in THF $(3.3 \mathrm{~mL})$ was added, the flask was rinsed with THF $(0.9 \mathrm{~mL})$. The resulting solution was stirred at $65{ }^{\circ} \mathrm{C}$ for $4 \mathrm{~h}$. The unpurified reaction mixture showed a d.r. of 7.2:1. The reaction mixture was then diluted with $\mathrm{Et}_{2} \mathrm{O}$, loaded on silica and the crude product purified by flash column chromatography (ethyl acetate/hexane $=0 \%$ to $8 \%$ ). The product 4 was obtained as a colorless oil ( $490 \mathrm{mg}, 80 \%, 90 \%$ ee).

${ }^{1} \mathbf{H}$ NMR $\left(500 \mathrm{MHz}, \mathrm{CDCl}_{3}\right) \delta: 7.33-7.27\left(\mathrm{~m}, 2 \mathrm{H}, \mathrm{C}_{\text {meta }}-\mathrm{H}\right), 7.01\left(\mathrm{tt}, J=7.4,1.1 \mathrm{~Hz}, 1 \mathrm{H}, \mathrm{C}_{\mathrm{para}}-\mathrm{H}\right)$, $6.94-6.87\left(\mathrm{~m}, 2 \mathrm{H}, \mathrm{C}_{\text {ortho }}-\mathrm{H}\right), 6.21\left(\mathrm{ddt}, J=15.9,7.6,2.5 \mathrm{~Hz}, 1 \mathrm{H}, \mathrm{C}\left(1^{\circ}\right)-\mathrm{H}\right), 5.92(\mathrm{dt}, J=5.8,1.8 \mathrm{~Hz}$, $1 \mathrm{H}, \mathrm{C}(6)-\mathrm{H}), 5.80-5.69\left(\mathrm{~m}, 2 \mathrm{H}, \mathrm{C}\left(2^{\circ}\right)-\mathrm{H}\right.$ and $\left.\mathrm{C}(5)-\mathrm{H}\right), 5.16(\mathrm{dq}, J=5.7,1.4 \mathrm{~Hz}, 1 \mathrm{H}, \mathrm{C}(6 \mathrm{a})-\mathrm{H}), 4.46$ $(\mathrm{d}, J=5.7 \mathrm{~Hz}, 1 \mathrm{H}, \mathrm{C}(3 \mathrm{a})-\mathrm{H}), 4.18\left(\mathrm{t}, J=11.5 \mathrm{~Hz}, 2 \mathrm{H}, \mathrm{C}\left(4^{\circ}\right)-\mathrm{H}_{2}\right), 3.54(\mathrm{~d}, J=6.6 \mathrm{~Hz}, 1 \mathrm{H}, \mathrm{C}(4)-\mathrm{H})$, $1.43\left(\mathrm{~s}, 3 \mathrm{H}, \mathrm{CH}_{3}\right), 1.34\left(\mathrm{~s}, 3 \mathrm{H}, \mathrm{CH}_{3}\right)$.

${ }^{13} \mathbf{C}$ NMR (126 MHz, $\left.\mathrm{CDCl}_{3}\right) \delta: 158.1(\mathrm{Cq}), 137.3\left(\mathrm{t}, J=8.9 \mathrm{~Hz}, \mathrm{C}\left(1^{\circ}\right)\right), 133.8(\mathrm{C}(5)), 132.9(\mathrm{C}(6))$, 129.8 ( $\left.\mathrm{C}_{\text {meta }}\right), 123.8$ (t, J = 25.1 Hz, C(2 $\left.\left.2^{\circ}\right)\right), 122.0$ ( $\left.\mathrm{C}_{\text {para }}\right), 118.3$ (t, $\left.J=240.3 \mathrm{~Hz}, \mathrm{C}\left(3^{\circ}\right)\right), 114.9$ ( $\left.\mathrm{C}_{\text {ortho }}\right)$, $110.6(\mathrm{C}(2)), 85.0(\mathrm{C}(6 \mathrm{a})), 83.1(\mathrm{C}(3 \mathrm{a})), 69.6\left(\mathrm{t}, J=35.1 \mathrm{~Hz}, \mathrm{C}\left(4^{\circ}\right)\right), 53.8(\mathrm{C}(4)), 27.5\left(\mathrm{CH}_{3}\right), 25.8$ $\left(\mathrm{CH}_{3}\right)$.

${ }^{19}$ F NMR $\left(377 \mathrm{MHz}, \mathrm{CDCl}_{3},{ }^{1} \mathrm{H}\right.$ decoupled) $\delta:-102.69$ (d, $\left.J=257.7 \mathrm{~Hz}\right),-103.46$ (d, $\left.J=257.9 \mathrm{~Hz}\right)$.

FT-IR $\left(\mathrm{CHCl}_{3}\right.$ film) cm $\mathrm{cm}^{-1}: 2987$ (w), 1599 (w), 1496 (m), 1287 (m), 1210 (m), 1160 (m), 1050 (s), 754 (m).

HRMS (ESI) m/z: [M + Na $]^{+}$Calcd for $\mathrm{C}_{18} \mathrm{H}_{20} \mathrm{O}_{3} \mathrm{~F}_{2} \mathrm{Na}$ 345.1273; Found 345.1273.

SFC Chiralpak ${ }^{\circledR} \mathrm{IF} ; 1500 \mathrm{psi}, 30{ }^{\circ} \mathrm{C}$; flow: $1.5 \mathrm{~mL} / \mathrm{min}$; from $1 \%$ to $30 \% \mathrm{MeOH}$ in $5 \mathrm{~min}$; 95.0:5.0 er (major enantiomer $t_{R}=1.66 \mathrm{~min}$; minor enantiomer $t_{R}=1.76 \mathrm{~min}$ ).

$[\boldsymbol{\alpha}]^{25}{ }_{\mathbf{D}}=-131.8\left(\mathrm{c}=0.5, \mathrm{CHCl}_{3}\right)$. 


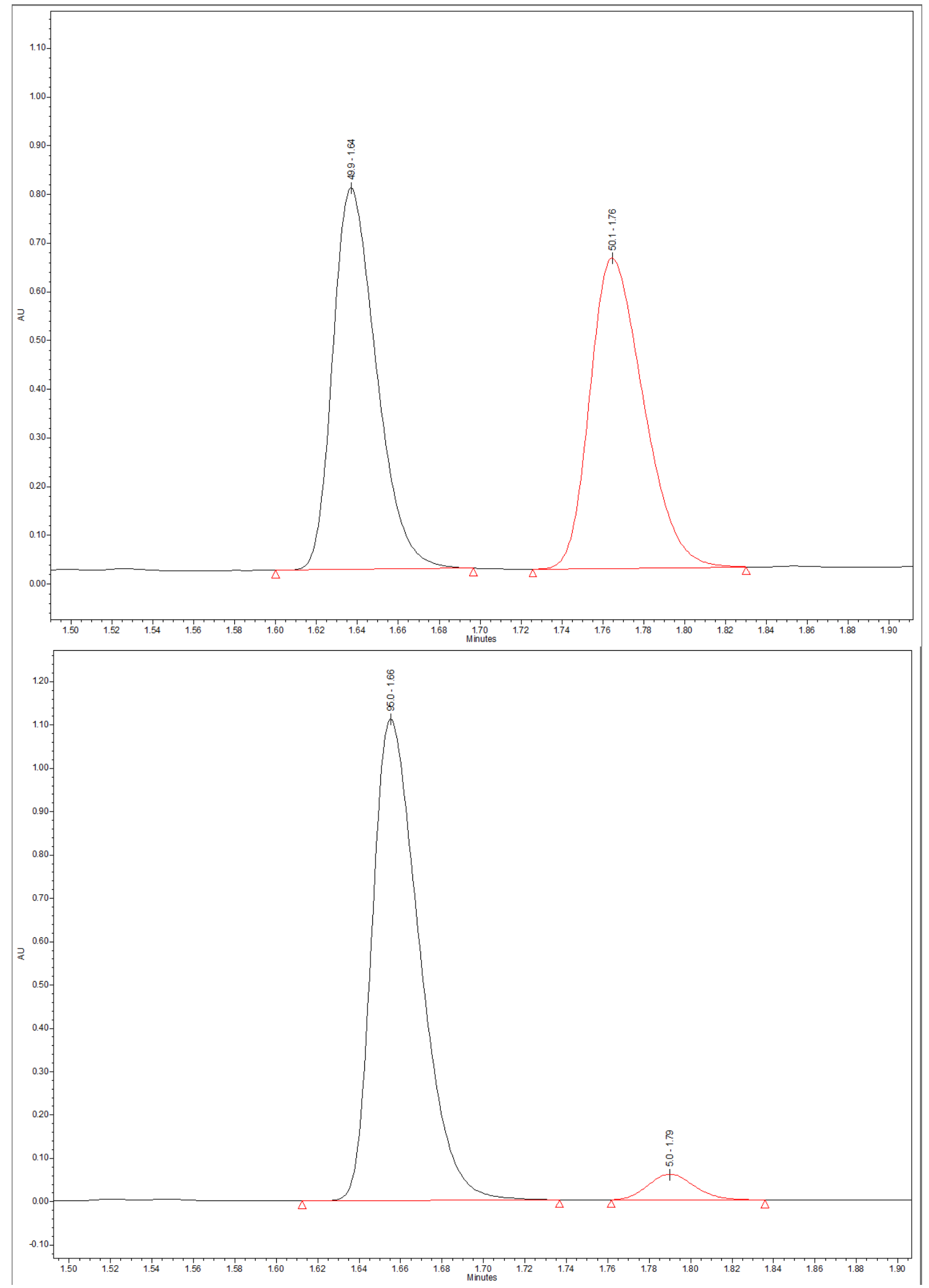

Figure S1: SFC traces of the racemic ( \pm )-4 (top) and enantioenriched (-)-4 (bottom). 
(3aS,4R,6aR)-4-((E)-3,3-difluoro-4-phenoxybut-1-en-1-yl)-2,2-dimethyl-3a,6a-dihydro-4Hcyclopenta[d][1,3]dioxole (S12)

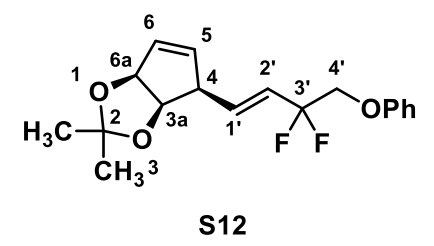

Compound S12 was isolated as a by-product of the above describe coupling reaction between allylchloride $( \pm)-2$ and boronic acid $\mathbf{3}$ as a colorless oil $(61 \mathrm{mg}, 10 \%)$. The ee of the minor diastereomer was not determined.

${ }^{1} \mathbf{H}$ NMR $\left(500 \mathrm{MHz}, \mathrm{CDCl}_{3}\right) \delta: 7.34-7.26\left(\mathrm{~m}, 2 \mathrm{H}, \mathrm{C}_{\text {meta }}-\mathrm{H}\right), 7.04-6.97$ $\left(\mathrm{m}, 1 \mathrm{H}, \mathrm{C}_{\text {para }}-\mathrm{H}\right), 6.97-6.88\left(\mathrm{~m}, 2 \mathrm{H}, \mathrm{C}_{\text {ortho }}-\mathrm{H}\right), 6.32$ (ddt, $\left.J=16.0,8.2,2.5 \mathrm{~Hz}, 1 \mathrm{H}, \mathrm{C}\left(1^{\circ}\right)-\mathrm{H}\right), 5.90$ (dt, $J=5.8,2.2 \mathrm{~Hz}, 1 \mathrm{H}, \mathrm{C}(6)-\mathrm{H}), 5.82\left(\mathrm{dtd}, J=15.9,11.1,1.1 \mathrm{~Hz}, 1 \mathrm{H}, \mathrm{C}\left(2^{\circ}\right)-\mathrm{H}\right), 5.71-5.66(\mathrm{~m}, 1 \mathrm{H}, \mathrm{C}(5)-$ H), $5.13(\mathrm{~d}, J=5.6 \mathrm{~Hz}, 1 \mathrm{H}, \mathrm{C}(6 \mathrm{a})-\mathrm{H}), 4.77(\mathrm{t}, J=5.8 \mathrm{~Hz}, 1 \mathrm{H}, \mathrm{C}(3 \mathrm{a})-\mathrm{H}), 4.21(\mathrm{t}, J=11.6 \mathrm{~Hz}, 2 \mathrm{H}$, $\left.\mathrm{C}\left(4^{\circ}\right)-\mathrm{H}_{2}\right), 3.44-3.37$ (m, $\left.1 \mathrm{H}, \mathrm{C}(4)-\mathrm{H}\right), 1.36\left(\mathrm{~s}, 3 \mathrm{H}, \mathrm{CH}_{3}\right), 1.34$ (s, 3H, $\left.\mathrm{CH}_{3}\right)$.

${ }^{13}$ C NMR (126 MHz, $\left.\mathrm{CDCl}_{3}\right) \delta: 158.2\left(\mathrm{C}_{\mathrm{q}}\right), 135.2\left(\mathrm{t}, J=9.1 \mathrm{~Hz}, \mathrm{C}\left(1^{\circ}\right)\right), 134.3(\mathrm{C}(5)), 132.3(\mathrm{C}(6))$, $129.7\left(\mathrm{C}_{\text {meta }}\right), 124.0\left(\mathrm{t}, J=25.3 \mathrm{~Hz}, \mathrm{C}\left(2^{\circ}\right)\right), 121.9\left(\mathrm{C}_{\text {para }}\right), 118.4\left(\mathrm{t}, J=240.2 \mathrm{~Hz}, \mathrm{C}\left(3^{\circ}\right)\right), 115.0\left(\mathrm{C}_{\text {meta }}\right)$, $111.1(\mathrm{C}(2)), 85.6(\mathrm{C}(6 \mathrm{a})), 79.5(\mathrm{C}(3 \mathrm{a})), 69.8\left(\mathrm{t}, J=34.6 \mathrm{~Hz}, \mathrm{C}\left(4^{\circ}\right)\right), 50.2(\mathrm{C}(4)), 27.4\left(\mathrm{CH}_{3}\right), 26.2$ $\left(\mathrm{CH}_{3}\right)$.

${ }^{19}$ F NMR (377 MHz, $\mathrm{CDCl}_{3},{ }^{1} \mathrm{H}$ decoupled) $\delta:-102.93,-102.95$.

FT-IR $\left(\mathrm{CHCl}_{3}\right.$ film) cm $\mathrm{cm}^{-1}: 2987$ (w), 1599 (w), 1497 (m), 1249 (m), 1161 (m), 1059 (vs), 755 (m).

HRMS (ESI) m/z: [M + Na] $]^{+}$Calcd for $\mathrm{C}_{18} \mathrm{H}_{20} \mathrm{O}_{3} \mathrm{~F}_{2} \mathrm{Na}$ 345.1273; Found 345.1273. 


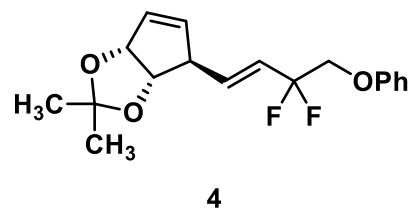

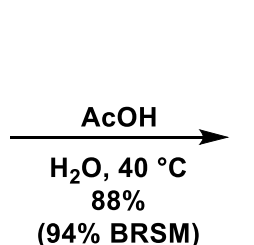

Diol S13 was prepared adopting procedure by F. W. Goetzke et al $^{[3]}$

A solution of acetonide $4(764 \mathrm{mg})$ in $\mathrm{AcOH}(5.2 \mathrm{~mL})$ and water $(2.3 \mathrm{~mL})$ was stirred for $19 \mathrm{~h}$ at 40 ${ }^{\circ} \mathrm{C}$. The reaction mixture was then concentrated under reduced pressure. Purification by gradient flash chromatography (ethyl acetate/hexane $=50 \%$ to $60 \%$ ) afforded diol $\mathbf{S 1 3}$ as a white solid $(588 \mathrm{mg}$, $88 \%, 94 \%$ brsm).

${ }^{1} \mathbf{H}$ NMR $\left(500 \mathrm{MHz}, \mathrm{CDCl}_{3}\right) \delta: 7.34-7.26\left(\mathrm{~m}, 2 \mathrm{H}, \mathrm{C}_{\text {meta }}-\mathrm{H}\right), 7.01\left(\mathrm{tt}, J=7.3,1.1 \mathrm{~Hz}, 1 \mathrm{H}, \mathrm{C}_{\mathrm{para}}-\mathrm{H}\right)$, $6.95-6.89\left(\mathrm{~m}, 2 \mathrm{H}, \mathrm{C}_{\text {ortho-H) }}\right.$, 6.25 (ddt, $\left.J=15.8,7.6,2.5 \mathrm{~Hz}, 1 \mathrm{H}, \mathrm{C}\left(1^{\circ}\right)-\mathrm{H}\right), 5.96$ (dt, $J=6.1,2.2 \mathrm{~Hz}$, $1 \mathrm{H}, \mathrm{C}(3)-\mathrm{H}), 5.89(\mathrm{dd}, J=6.1,2.0 \mathrm{~Hz}, 1 \mathrm{H}, \mathrm{C}(4)-\mathrm{H}), 5.81\left(\mathrm{dtd}, J=15.9,11.2,1.3 \mathrm{~Hz}, 1 \mathrm{H}, \mathrm{C}\left(2^{\circ}\right)-\mathrm{H}\right)$, $4.64-4.58(\mathrm{~m}, 1 \mathrm{H}, \mathrm{C}(2)-\mathrm{H}), 4.19\left(\mathrm{t}, J=11.6 \mathrm{~Hz}, 2 \mathrm{H}, \mathrm{C}\left(4^{\circ}\right)-\mathrm{H}_{2}\right), 3.97(\mathrm{q}, J=5.6 \mathrm{~Hz}, 1 \mathrm{H}, \mathrm{C}(1)-\mathrm{H})$, $3.38(\mathrm{~m}, 1 \mathrm{H}, \mathrm{C}(5)-\mathrm{H}), 2.70(\mathrm{~d}, J=7.1 \mathrm{~Hz}, 1 \mathrm{H}, \mathrm{OH}), 2.15(\mathrm{~d}, J=6.2 \mathrm{~Hz}, 1 \mathrm{H}, \mathrm{OH})$.

${ }^{13}$ C NMR (126 MHz, $\left.\mathrm{CDCl}_{3}\right) \delta: 158.1\left(\mathrm{C}_{\mathrm{q}}\right), 137.1\left(\mathrm{t}, J=8.9 \mathrm{~Hz}, \mathrm{C}\left(1^{\prime}\right)\right), 136.1(\mathrm{C}(4)), 132.7(\mathrm{C}(3))$, $129.8\left(\mathrm{C}_{\text {meta }}\right), 123.7$ (t, $\left.J=25.1 \mathrm{~Hz}, \mathrm{C}\left(2^{\circ}\right)\right), 122.0\left(\mathrm{C}_{\mathrm{para}}\right), 118.3$ (t, $\left.J=240.3 \mathrm{~Hz}, \mathrm{C}\left(3^{\circ}\right)\right), 115.0\left(\mathrm{C}_{\text {ortho }}\right)$, $76.9(\mathrm{C}(1)), 75.0(\mathrm{C}(2)), 69.6\left(\mathrm{t}, J=34.8 \mathrm{~Hz}, \mathrm{C}\left(4^{\circ}\right)\right), 53.7(\mathrm{C}(5))$.

${ }^{19}$ F NMR (377 MHz, $\mathrm{CDCl}_{3},{ }^{1} \mathrm{H}$ decoupled) $\delta:-102.66(\mathrm{~d}, J=257.2 \mathrm{~Hz}),-103.38(\mathrm{~d}, J=256.5 \mathrm{~Hz})$.

FT-IR $\left(\mathrm{CHCl}_{3}\right.$ film) cm $\mathrm{cm}^{-1}: 3360$ (br. m), 1599 (m), 1496 (s), 1248 (s), 1163 (m), 1065 (s), 754 (s).

HRMS (ESI) m/z: [M + Na $]^{+}$Calcd for $\mathrm{C}_{15} \mathrm{H}_{16} \mathrm{O}_{3} \mathrm{~F}_{2} \mathrm{Na}$ 305.0960; Found 305.0961.

m.p. $45-48^{\circ} \mathrm{C}$.

$[\alpha]^{25}=-152.9\left(\mathrm{c}=0.5, \mathrm{CHCl}_{3}\right)$. 
(3aS,4S,6aR)-4-((E)-3,3-difluoro-4-phenoxybut-1-en-1-yl)-3a,6a-dihydro-4H-cyclopenta[d][1,3]dioxol-2-one (5)
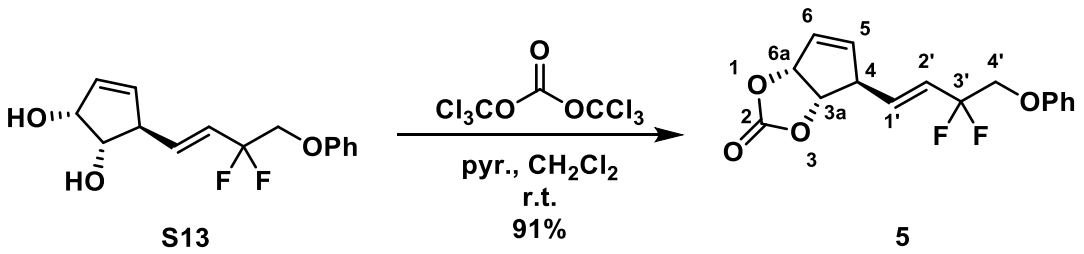

A solution of triphosgene (399 mg, $1.34 \mathrm{mmol}, 1.10$ equiv.) in $\mathrm{CH}_{2} \mathrm{Cl}_{2}(4.0 \mathrm{~mL})$ was added to a solution of diol $\mathbf{S 1 3}$ (353 mg, $1.21 \mathrm{mmol}, 1.00$ equiv.) and pyridine (590 $\mu \mathrm{L}, 7.3 \mathrm{mmol}, 6.0$ equiv.) in $\mathrm{CH}_{2} \mathrm{Cl}_{2}(7.5 \mathrm{~mL})$ at r.t. After stirring for $20 \mathrm{~min}$, the reaction mixture was directly loaded onto silica and purification by gradient flash chromatography (ethyl acetate/hexane $=10 \%$ to $25 \%$ ) afforded the product 5 as a colorless oil (352 $\mathrm{mg}, 91 \%)$.

${ }^{1} \mathbf{H}$ NMR $\left(400 \mathrm{MHz}, \mathrm{CDCl}_{3}\right) \delta: 7.36-7.26\left(\mathrm{~m}, 2 \mathrm{H}, \mathrm{C}_{\text {meta }}-\mathrm{H}\right), 7.02\left(\mathrm{tt}, J=7.3,1.1 \mathrm{~Hz}, 1 \mathrm{H}, \mathrm{C}_{\mathrm{para}}-\mathrm{H}\right)$, $6.96-6.86\left(\mathrm{~m}, 2 \mathrm{H}, \mathrm{C}_{\text {ortho }}-\mathrm{H}\right), 6.20$ (ddt, $\left.J=15.9,7.3,2.4 \mathrm{~Hz}, 1 \mathrm{H}, \mathrm{C}\left(1^{\circ}\right)-\mathrm{H}\right), 6.14-6.07(\mathrm{~m}, 1 \mathrm{H}, \mathrm{C}(5)-$ H), $6.03(\mathrm{dt}, J=5.8,1.8 \mathrm{~Hz}, 1 \mathrm{H}, \mathrm{C}(6)-\mathrm{H}), 5.78\left(\mathrm{dtd}, J=15.9,11.0,1.4 \mathrm{~Hz}, 1 \mathrm{H}, \mathrm{C}\left(2^{\circ}\right)-\mathrm{H}\right), 5.59$ (dtd, $J$ $=6.6,1.6,0.9 \mathrm{~Hz}, 1 \mathrm{H}, \mathrm{C}(6 \mathrm{a})-\mathrm{H}), 4.87(\mathrm{dt}, J=6.5,0.9 \mathrm{~Hz}, 1 \mathrm{H}, \mathrm{C}(3 \mathrm{a})-\mathrm{H}), 4.19(\mathrm{t}, J=11.3 \mathrm{~Hz}, 2 \mathrm{H}$, $\left.\mathrm{C}\left(4^{\circ}\right)-\mathrm{H}\right), 3.84-3.76(\mathrm{~m}, 1 \mathrm{H}, \mathrm{C}(4)-\mathrm{H})$.

${ }^{13}$ C NMR (101 MHz, $\left.\mathrm{CDCl}_{3}\right) \delta: 157.8\left(\mathrm{C}_{\mathrm{q}}\right), 154.1(\mathrm{C}(2)), 137.8(\mathrm{C}(5)), 134.4\left(\mathrm{t}, J=8.9 \mathrm{~Hz}, \mathrm{C}\left(1^{\circ}\right)\right)$, $129.8\left(\mathrm{C}_{\text {meta }}\right), 129.5(\mathrm{C}(6)), 125.8\left(\mathrm{t}, J=25.4 \mathrm{~Hz}, \mathrm{C}\left(2^{\prime}\right)\right), 122.2\left(\mathrm{C}_{\text {para }}\right), 118.0\left(\mathrm{t}, J=240.8 \mathrm{~Hz}, \mathrm{C}\left(3^{\circ}\right)\right)$, 114.9 ( $\left.\mathrm{C}_{\text {ortho }}\right), 84.6$ (C(6a)), 82.1 (t, $\left.J=2.1 \mathrm{~Hz}, \mathrm{C}(3 \mathrm{a})\right), 69.4$ (t, $\left.J=35.3 \mathrm{~Hz}, \mathrm{C}\left(4^{\circ}\right)\right), 53.2$ (C(4)).

${ }^{19}$ F NMR (376 MHz, $\mathrm{CDCl}_{3},{ }^{1} \mathrm{H}$ decoupled) $\delta:-103.19(\mathrm{~d}, J=259.7 \mathrm{~Hz}),-103.97(\mathrm{~d}, J=259.2 \mathrm{~Hz})$.

FT-IR $\left(\mathrm{CHCl}_{3}\right.$ film) cm ${ }^{-1}: 1791$ (s), 1598 (w), 1495 (m), 1248 (m), 1157 (s), 1047 (s), 7570 (s).

HRMS (ESI) m/z: [M + Na] $]^{+}$Calcd for $\mathrm{C}_{16} \mathrm{H}_{14} \mathrm{O}_{4} \mathrm{~F}_{2} \mathrm{Na} 331.0752$; Found 331.0574.

$[\alpha]^{25}=-170.7\left(\mathrm{c}=0.5, \mathrm{CHCl}_{3}\right)$. 
Diethyl 2-((1S,4R,5R)-5-((E)-3,3-difluoro-4-phenoxybut-1-en-1-yl)-4-hydroxycyclopent-2-en-1yl)malonate (6)
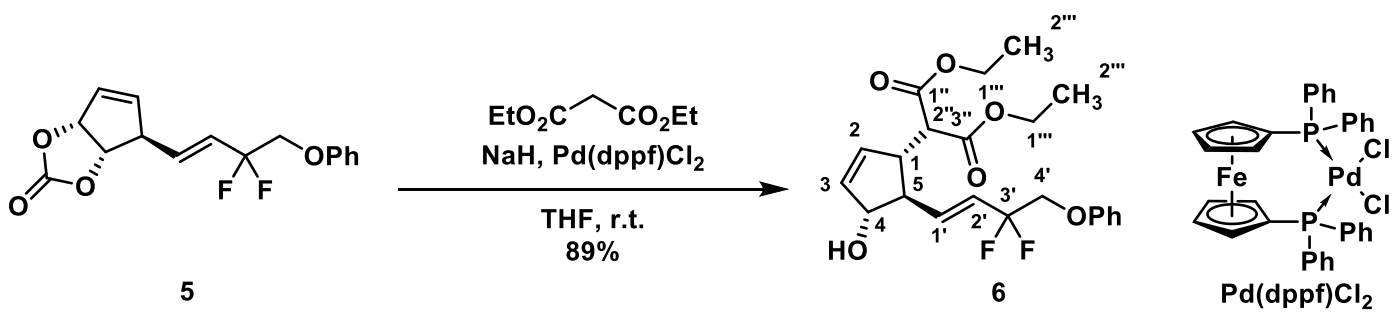

Compound 6 was prepared adopting procedure by H. L. Sebahar et al. ${ }^{[10]}$

A solution of malonate enolate, prepared by addition of diethyl malonate $(480 \mu \mathrm{L}, 3.2 \mathrm{mmol}, 3.5$ equiv.) to mixture of $\mathrm{NaH}$ ( $60 \%$ in mineral oil, washed with pentane; $109 \mathrm{mg}, 2.72 \mathrm{mmol}, 3.00$ equiv.) in THF $(3.0 \mathrm{~mL})$, was added to a solution of carbonate 5 (280 mg, $0.908 \mathrm{mmol}, 1.00$ equiv.) and [1, 1'bis(diphenylphosphino)ferrocene]dichloropalladium ( $\left.\mathrm{Pd}(\mathrm{dppf}) \mathrm{Cl}_{2}\right)(19.9 \mathrm{mg}, 27.3 \mu \mathrm{mol}, 0.03$ equiv.) in THF $(10 \mathrm{~mL})$ at r.t.. After stirring for $1 \mathrm{~h}$, the reaction mixture was poured onto an aq. sat. sol. of $\mathrm{NH}_{4} \mathrm{Cl}(30 \mathrm{~mL})$ and was extracted with ethyl acetate $(3 \times 30 \mathrm{~mL})$. The combined organic phases were dried over $\mathrm{Na}_{2} \mathrm{SO}_{4}$, and concentrated under reduced pressure. Purification by gradient flash column chromatography (ethyl acetate/hexane $=10 \%$ to $40 \%$ ) afforded 6 as a colorless oil ( $342 \mathrm{mg}, 89 \%$ ).

${ }^{1}$ H NMR $\left(400 \mathrm{MHz}, \mathrm{CDCl}_{3}\right) \delta: 7.34-7.25\left(\mathrm{~m}, 2 \mathrm{H}, \mathrm{C}_{\text {meta }}-\mathrm{H}\right), 7.04-6.96\left(\mathrm{~m}, 1 \mathrm{H}, \mathrm{C}_{\mathrm{para}}-\mathrm{H}\right), 6.96-6.88$ (m, $\left.2 \mathrm{H}, \mathrm{C}_{\text {ortho }}-\mathrm{H}\right), 6.17$ (ddt, $\left.J=15.7,9.3,2.5 \mathrm{~Hz}, 1 \mathrm{H}, \mathrm{C}\left(1^{\circ}\right)-\mathrm{H}\right), 5.92-5.84(\mathrm{~m}, 2 \mathrm{H}, \mathrm{C}(2)-\mathrm{H}$ and $\mathrm{C}(3)-$ $\mathrm{H}), 5.88-5.74\left(\mathrm{~m}, 1 \mathrm{H}, \mathrm{C}\left(2^{\circ}\right)-\mathrm{H}\right), 4.53(\mathrm{dt}, J=5.4,2.8 \mathrm{~Hz}, 1 \mathrm{H}, \mathrm{C}(4)-\mathrm{H}), 4.29-4.07$ (m, 6H, C(4 $)-\mathrm{H}_{2}$ and $\left.\mathrm{C}\left(1^{\prime \prime c}\right)-\mathrm{H}_{2}\right), 3.47\left(\mathrm{~d}, J=6.7 \mathrm{~Hz}, 1 \mathrm{H}, \mathrm{C}\left(2^{\prime c}\right)-\mathrm{H}\right), 3.13-3.04(\mathrm{~m}, 1 \mathrm{H}, \mathrm{C}(1)-\mathrm{H}), 2.66$ (dddd, $J=7.9$, $5.3,4.1,1.1 \mathrm{~Hz}, 1 \mathrm{H}, \mathrm{C}(5)-\mathrm{H}), 2.44(\mathrm{~d}, J=8.4 \mathrm{~Hz}, 1 \mathrm{H}, \mathrm{OH}), 1.25\left(\mathrm{t}, J=7.1 \mathrm{~Hz}, 3 \mathrm{H}, \mathrm{CH}_{3}\right), 1.24(\mathrm{t}, J=$ $\left.7.1 \mathrm{~Hz}, 3 \mathrm{H}, \mathrm{CH}_{3}\right)$.

${ }^{13} \mathrm{C}$ NMR (101 MHz, $\left.\mathrm{CDCl}_{3}\right) \delta: 168.4\left(\mathrm{C}\left(1^{\circ} \mathrm{c}\right)\right), 168.2\left(\mathrm{C}\left(3^{\circ} \mathrm{c}\right)\right), 158.1\left(\mathrm{C}_{\mathrm{q}}\right), 138.2\left(\mathrm{t}, J=9.0 \mathrm{~Hz}, \mathrm{C}\left(1^{\circ}\right)\right)$, $134.5(\mathrm{C}(2)), 133.5(\mathrm{C}(3)), 129.7\left(\mathrm{C}_{\text {meta }}\right), 123.7\left(\mathrm{t}, J=25.1 \mathrm{~Hz}, \mathrm{C}\left(2^{\circ}\right)\right), 122.0\left(\mathrm{C}_{\text {para }}\right), 118.2(\mathrm{t}, J=239.0$

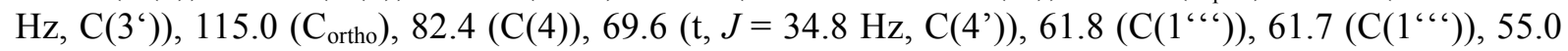
$\left(\mathrm{C}\left(2^{\text {‘ }}\right)\right), 54.3(\mathrm{C}(5)), 49.6(\mathrm{C}(1)), 14.2\left(\mathrm{C}\left(2^{\text {‘ }}\right)\right), 14.1\left(\mathrm{C}\left(2^{\text {‘“ }}\right)\right)$.

${ }^{19}$ F NMR (376 MHz, $\mathrm{CDCl}_{3},{ }^{1} \mathrm{H}$ decoupled) $\delta:-102.03(\mathrm{~d}, J=257.0 \mathrm{~Hz}),-103.78(\mathrm{~d}, J=257.1 \mathrm{~Hz})$.

FT-IR ( $\mathrm{CHCl}_{3}$ film) cm ${ }^{-1}: 3509$ (br, w), 1729 (s), 1599 (w), 1496 (w), 1249 (s), 1177 (s), 1159 (s), $1033(\mathrm{~m}), 756(\mathrm{~m})$.

HRMS (ESI) m/z: $[\mathrm{M}+\mathrm{Na}]^{+}$Calcd for $\mathrm{C}_{22} \mathrm{H}_{26} \mathrm{O}_{6} \mathrm{~F}_{2} \mathrm{Na}$ 447.1590; Found 447.1588.

$[\alpha]^{25}=+20.3\left(\mathrm{c}=0.5, \mathrm{CHCl}_{3}\right)$. 


\section{Diethyl 2-((4S,5S)-4-((E)-3,3-difluoro-4-phenoxybut-1-en-1-yl)-5-hydroxycyclopent-2-en-1- yl)malonate (S14)}

Compound S14 was isolated as a by-product of allylic substitution reaction between carbonate $\mathbf{5}$ and malonate anion as a colorless oil (33.4 $\mathrm{mg}, 9 \%)$.

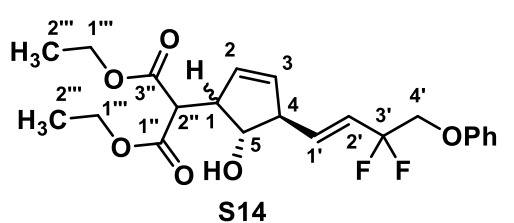

${ }^{1} \mathbf{H}$ NMR $\left(500 \mathrm{MHz},\left(\mathrm{CD}_{3}\right)_{2} \mathrm{CO}\right) \delta: 7.36-7.28\left(\mathrm{~m}, 2 \mathrm{H}, \mathrm{C}_{\text {meta }}-\mathrm{H}\right)$, $7.04-6.96\left(\mathrm{~m}, 3 \mathrm{H}, \mathrm{C}_{\text {ortho }}-\mathrm{H}\right.$ and $\left.\mathrm{C}_{\text {para }}-\mathrm{H}\right), 6.30$ (ddt, $J=16.0,7.3$, $\left.2.5 \mathrm{~Hz}, 1 \mathrm{H}, \mathrm{C}\left(1^{\circ}\right)-\mathrm{H}\right), 5.88$ (dtd, $J=16.0,11.2,1.4 \mathrm{~Hz}, 1 \mathrm{H}, \mathrm{C}\left(2^{\circ}\right)-$ $\mathrm{H}), 5.75(\mathrm{dt}, J=6.1,2.2 \mathrm{~Hz}, 1 \mathrm{H}, \mathrm{C}(3)-\mathrm{H}), 5.67(\mathrm{dt}, J=6.1,2.1 \mathrm{~Hz}$, $1 \mathrm{H}, \mathrm{C}(2)-\mathrm{H}), 4.33\left(\mathrm{t}, J=12.3 \mathrm{~Hz}, 2 \mathrm{H}, \mathrm{C}\left(4^{\circ}\right)-\mathrm{H}_{2}\right), 4.17(\mathrm{~m}, 4 \mathrm{H}$, $\mathrm{C}\left(1^{\prime c}\right.$ ) $\left.)-\mathrm{H}_{2}\right), 4.04$ (q, $\left.J=6.0 \mathrm{~Hz}, 1 \mathrm{H}, \mathrm{C}(5)-\mathrm{H}\right), 3.47$ (d, $\left.J=7.9 \mathrm{~Hz}, 1 \mathrm{H}, \mathrm{C}\left(2^{\prime c}\right)-\mathrm{H}\right), 3.37-3.29$ (m, $1 \mathrm{H}$, C(4)-H), 3.20 (ddq, $J=8.2,6.3,2.2 \mathrm{~Hz}, 1 \mathrm{H}, \mathrm{C}(1)-\mathrm{H}), 1.23$ (t, $\left.J=7.0 \mathrm{~Hz}, 3 \mathrm{H}, \mathrm{CH}_{3}\right), 1.22$ (t, $J=7.0$ $\left.\mathrm{Hz}, 3 \mathrm{H}, \mathrm{CH}_{3}\right)$.

${ }^{13} \mathrm{C}$ NMR (126 MHz, $\left.\left(\mathrm{CD}_{3}\right)_{2} \mathrm{CO}\right) \delta: 168.5\left(\mathrm{C}\left(3^{\circ ‘}\right)\right), 168.1\left(\mathrm{C}\left(1^{\prime c}\right)\right), 158.3\left(\mathrm{C}_{\mathrm{q}}\right), 138.7(\mathrm{t}, J=9.0 \mathrm{~Hz}$, $\left.\mathrm{C}\left(1^{\circ}\right)\right), 131.4(\mathrm{C}(3)), 131.1(\mathrm{C}(2)), 129.6\left(\mathrm{C}_{\text {meta }}\right), 123.0$ (t, $\left.J=25.2 \mathrm{~Hz}, \mathrm{C}\left(2^{\circ}\right)\right), 121.5\left(\mathrm{C}_{\mathrm{para}}\right), 118.8$ (t, $J$ $\left.=239.1 \mathrm{~Hz}, \mathrm{C}\left(3^{\circ}\right)\right), 114.8\left(\mathrm{C}_{\text {ortho }}\right), 80.8(\mathrm{t}, J=1.9 \mathrm{~Hz}, \mathrm{C}(5)), 69.1$ (t, $\left.J=33.3 \mathrm{~Hz}, \mathrm{C}\left(4^{\circ}\right)\right), 61.1\left(\mathrm{C}\left(1^{\prime *}\right)\right)$, $61.0\left(\mathrm{C}\left(1^{، “}\right)\right), 55.6(\mathrm{C}(4)), 54.4\left(\mathrm{C}\left(2^{،}\right)\right), 53.2(\mathrm{C}(1)), 13.6\left(\mathrm{C}\left(2^{، “}\right)\right), 13.5\left(\mathrm{C}\left(2^{، “}\right)\right)$.

${ }^{19}$ F NMR $\left(377 \mathrm{MHz},\left(\mathrm{CD}_{3}\right)_{2} \mathrm{CO}\right) \delta:-102.76(\mathrm{~d}, J=255.9 \mathrm{~Hz}),-103.47(\mathrm{~d}, J=256.8 \mathrm{~Hz})$.

FT-IR $\left(\mathrm{CHCl}_{3}\right.$ film) cm ${ }^{-1}: 3511$ (br, w), 1725 (s), 1599 (w), 1496 (w), 1247 (s), 1160 (s), 1041 (m), $755(\mathrm{~m})$.

HRMS (ESI) m/z: [M + Na $]^{+}$Calcd for $\mathrm{C}_{22} \mathrm{H}_{26} \mathrm{O}_{6} \mathrm{~F}_{2} \mathrm{Na}$ 447.1590; Found 447.1585. 
2-((1S,4R,5R)-5-((E)-3,3-difluoro-4-phenoxybut-1-en-1-yl)-4-hydroxycyclopent-2-en-1-yl)malonic acid (S15)
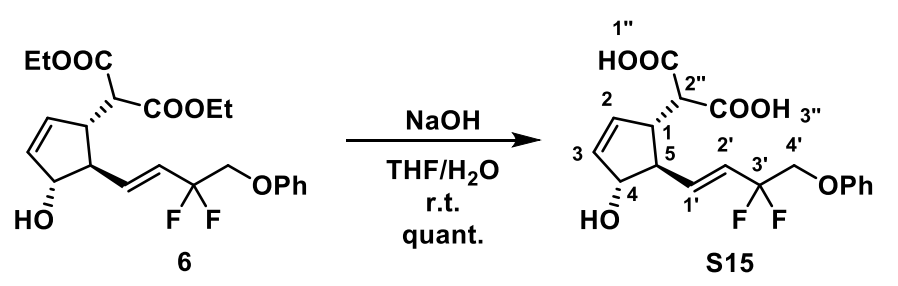

An aq. sol. of $\mathrm{NaOH}(1 \mathrm{M}, 14 \mathrm{~mL}, 14 \mathrm{mmol}, 16$ equiv.) was added to a solution of ester 6 (378 mg, $890 \mu \mathrm{mol}, 1.00$ equiv. $)$ in THF $(10 \mathrm{~mL})$ at r.t.. After stirring for $24 \mathrm{~h}$, the reaction mixture was acidified with $1 \mathrm{M}$ aq. solution of $\mathrm{HCl}(60 \mathrm{~mL})$ and extracted with ethyl acetate $(3 \times 60 \mathrm{~mL})$. The combined organic phases were washed with brine $(60 \mathrm{~mL})$, dried over $\mathrm{Na}_{2} \mathrm{SO}_{4}$, and concentrated under reduced pressure. The crude product $\mathbf{S 1 5}$ was obtained as a pale yellow oil (343 $\mathrm{mg},>95 \%)$ and was used in the next step without further purification.

${ }^{1} \mathbf{H}$ NMR $\left(400 \mathrm{MHz},\left(\mathrm{CD}_{3}\right)_{2} \mathrm{CO}\right) \delta: 11.30$ (br s, $\left.2 \mathrm{H}, \mathrm{COOH}\right), 7.35-7.26\left(\mathrm{~m}, 2 \mathrm{H}, \mathrm{C}_{\text {meta }}-\mathrm{H}\right), 7.05-6.94$ (m, $3 \mathrm{H}, \mathrm{C}_{\text {ortho }}-\mathrm{H}$ and $\left.\mathrm{C}_{\mathrm{para}}-\mathrm{H}\right), 6.34$ (ddt, $\left.J=15.8,8.9,2.6 \mathrm{~Hz}, 1 \mathrm{H}, \mathrm{C}\left(1^{\circ}\right)-\mathrm{H}\right), 5.96-5.80\left(\mathrm{~m}, 3 \mathrm{H}, \mathrm{C}\left(2^{\circ}\right)-\right.$ $\mathrm{H}, \mathrm{C}(2)-\mathrm{H}$ and $\mathrm{C}(3)-\mathrm{H}), 4.61(\mathrm{dq}, J=5.2,1.7 \mathrm{~Hz}, 1 \mathrm{H}, \mathrm{C}(4)-\mathrm{H}), 4.33$ (t, $\left.J=12.7 \mathrm{~Hz}, 2 \mathrm{H}, \mathrm{C}\left(4^{\circ}\right)-\mathrm{H}_{2}\right)$, 3.42 (d, $\left.J=7.8 \mathrm{~Hz}, 1 \mathrm{H}, \mathrm{C}\left(2^{\prime \prime}\right)-\mathrm{H}\right), 3.15-3.05$ (m, 1H, C(1)-H), 2.62 (dddd, $J=7.8,6.7,5.2,1.2 \mathrm{~Hz}$, $1 \mathrm{H}, \mathrm{C}(5)-\mathrm{H})$.

${ }^{13}$ C NMR (101 MHz, $\left.\left(\mathrm{CD}_{3}\right)_{2} \mathrm{CO}\right) \delta: 169.9\left(\mathrm{C}\left(1^{\circ /}\right)\right), 169.6\left(\mathrm{C}\left(3^{\circ}\right.\right.$ “ $\left.)\right), 159.3\left(\mathrm{C}_{\mathrm{q}}\right), 139.6(\mathrm{t}, J=9.2 \mathrm{~Hz}$, $\left.\mathrm{C}\left(1^{\circ}\right)\right), 135.8(\mathrm{C}(2)), 133.5(\mathrm{C}(3)), 130.4\left(\mathrm{C}_{\text {meta }}\right), 124.4$ (t, $\left.J=25.1 \mathrm{~Hz}, \mathrm{C}\left(2^{\circ}\right)\right), 122.4\left(\mathrm{C}_{\mathrm{para}}\right), 119.6$ (t, $J$ $\left.=238.2 \mathrm{~Hz}, \mathrm{C}\left(3^{\circ}\right)\right), 115.8\left(\mathrm{C}_{\text {ortho }}\right), 82.0(\mathrm{C}(4)), 67.0$ (t, $\left.J=32.2 \mathrm{~Hz}, \mathrm{C}\left(4^{\circ}\right)\right), 56.0(\mathrm{C}(5)), 55.9\left(\mathrm{C}\left(2^{\prime \prime}\right)\right)$, $49.6(\mathrm{C}(1))$.

${ }^{19}$ F NMR $\left(377 \mathrm{MHz},\left(\mathrm{CD}_{3}\right)_{2} \mathrm{CO},{ }^{1} \mathrm{H}\right.$ decoupled) $\delta$ : $-103.33(\mathrm{~d}, J=252.4 \mathrm{~Hz}),-104.22(\mathrm{~d}, J=252.4$ $\mathrm{Hz})$.

FT-IR $\left(\mathrm{CHCl}_{3}\right.$ film) cm $\mathrm{cm}^{-1}: 2918$ (br. w), 1714(s), 1599 (m), 1495 (m), 1248 (s), 1164 (m), 1054 (m), $756(\mathrm{~s})$.

HRMS (ESI) m/z: [M + Na] $]^{+}$Calcd for $\mathrm{C}_{18} \mathrm{H}_{18} \mathrm{O}_{6} \mathrm{~F}_{2} \mathrm{Na}$ 391.0964; Found 391.0963.

$[\alpha]^{25}=+14.8\left(\mathrm{c}=0.5, \mathrm{CHCl}_{3}\right)$. 
2-((1S,4R,5R)-5-((E)-3,3-difluoro-4-phenoxybut-1-en-1-yl)-4-hydroxycyclopent-2-en-1-yl)acetic acid (7)
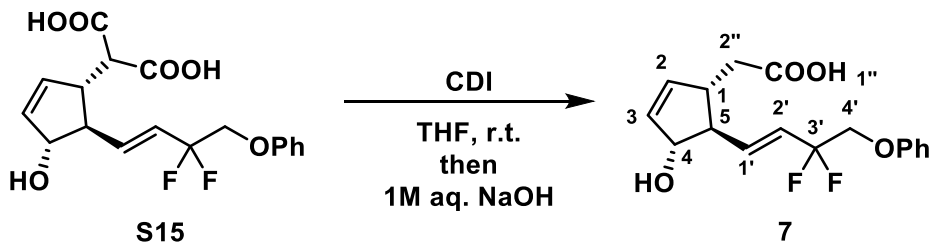

Compound 7 was prepared adopting procedure by D. Lafrance et al. ${ }^{[1]}$

1,1'-Carbonyldiimidazole (CDI) (402 mg, $2.23 \mathrm{mmol}, 2.50$ equiv.) was added to a solution of acid $\mathbf{S 1 5}$ (333 mg, $864 \mu \mathrm{mol}, 1.00$ equiv.) in THF $(18 \mathrm{~mL}$ ) at r.t.. After stirring the mixture for $3 \mathrm{~h}$, an aq. sol. of $1 \mathrm{M} \mathrm{NaOH}(7.8 \mathrm{~mL}, 7.8 \mathrm{mmol}, 8.7$ equiv.) was added and the reaction mixture stirred for $20 \mathrm{~h}$. The reaction mixture was then acidified with aq. $1 \mathrm{M} \mathrm{HCl}(30 \mathrm{~mL})$ and extracted with ethyl acetate $(3 \times 30$ $\mathrm{mL})$. The combined organic phases were washed with brine $(30 \mathrm{~mL})$, dried over $\mathrm{Na}_{2} \mathrm{SO}_{4}$, and concentrated under reduced pressure. The crude product 7 was obtained as a pale yellow oil (305 $\mathrm{mg}$, $>95 \%$ ) and used in the next step without further purification.

NOTE: The reaction can be monitored by TLC using $80 \% \mathrm{THF} / \mathrm{h}$ exane $+1 \% \mathrm{AcOH}$.

${ }^{1}$ H NMR $\left(400 \mathrm{MHz}, \mathrm{CDCl}_{3}\right) \delta: 7.35-7.24\left(\mathrm{~m}, 2 \mathrm{H}, \mathrm{C}_{\text {meta }}-\mathrm{H}\right), 7.04-6.97\left(\mathrm{~m}, 1 \mathrm{H}, \mathrm{C}_{\mathrm{para}}-\mathrm{H}\right), 6.97-6.88$ $\left(\mathrm{m}, 2 \mathrm{H}, \mathrm{C}_{\text {ortho }}-\mathrm{H}\right), 6.23$ (ddt, $\left.J=15.7,9.1,2.4 \mathrm{~Hz}, 1 \mathrm{H}, \mathrm{C}\left(1^{\circ}\right)-\mathrm{H}\right), 5.93-5.77\left(\mathrm{~m}, 3 \mathrm{H}, \mathrm{C}\left(2^{\circ}\right)-\mathrm{H}, \mathrm{C}(2)-\mathrm{H}\right.$ and $\mathrm{C}(3)-\mathrm{H}), 4.61(\mathrm{dq}, J=5.5,1.7 \mathrm{~Hz}, 1 \mathrm{H}, \mathrm{C}(4)-\mathrm{H}), 4.19\left(\mathrm{t}, J=11.6 \mathrm{~Hz}, 2 \mathrm{H}, \mathrm{C}\left(4^{\circ}\right)-\mathrm{H}_{2}\right), 2.81$ (dtd, $J=$ 8.2, 6.3, $1.9 \mathrm{~Hz}, 1 \mathrm{H}, \mathrm{C}(1)-\mathrm{H}), 2.47$ (dd, $\left.J=15.8,6.1 \mathrm{~Hz}, 1 \mathrm{H}, \mathrm{C}\left(2^{\prime \prime}\right)-\mathrm{H}\right), 2.40$ (dd, $J=15.8,8.0 \mathrm{~Hz}, 1 \mathrm{H}$, $\mathrm{C}(2$ “')-H), $2.38-2.30$ (m, 1H, C(5)-H).

${ }^{13} \mathrm{C}$ NMR (101 MHz, $\left.\mathrm{CDCl}_{3}\right) \delta 177.6\left(\mathrm{C}\left(1^{\circ *}\right)\right), 158.1\left(\mathrm{C}_{\mathrm{q}}\right), 137.6\left(\mathrm{t}, J=9.0 \mathrm{~Hz}, \mathrm{C}\left(1^{\prime}\right)\right), 135.2(\mathrm{C}(2))$, $133.6(\mathrm{C}(3)), 129.8\left(\mathrm{C}_{\text {meta }}\right), 124.5\left(\mathrm{t}, J=25.0 \mathrm{~Hz}, \mathrm{C}\left(2^{\circ}\right)\right), 122.0\left(\mathrm{C}_{\mathrm{para}}\right), 118.2\left(\mathrm{t}, J=240.6 \mathrm{~Hz}, \mathrm{C}\left(3^{\circ}\right)\right)$, 115.0 ( $\left.\mathrm{C}_{\text {ortho }}\right), 82.0(\mathrm{C}(4)), 69.5$ (t, $\left.J=34.6 \mathrm{~Hz}, \mathrm{C}\left(4^{6}\right)\right), 57.7$ (C(5)), 45.9 (C(1)), $38.4\left(\mathrm{C}\left(2^{،}\right.\right.$ “)).

${ }^{19}$ F NMR (377 MHz, $\mathrm{CDCl}_{3},{ }^{1} \mathrm{H}$ decoupled) $\delta:-102.50(\mathrm{~d}, J=256.8 \mathrm{~Hz}),-103.31(\mathrm{~d}, J=256.5 \mathrm{~Hz})$.

FT-IR ( $\mathrm{CHCl}_{3}$ film) cm $\mathrm{cm}^{-1}: 2927$ (br. w.), 1710 (s), 1599(m), 1496 (m), 1248 (s), 1161 (s), 1054 (s), $754(s)$.

HRMS (ESI) m/z: [M + Na] $]^{+}$Calcd for $\mathrm{C}_{17} \mathrm{H}_{18} \mathrm{O}_{4} \mathrm{~F}_{2} \mathrm{Na}$ 347.1065; Found 347.1065.

$[\alpha]^{25}{ }_{\mathrm{D}}=-2.5\left(\mathrm{c}=0.5, \mathrm{CHCl}_{3}\right)$. 


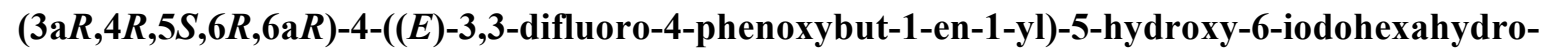
2H-cyclopenta[b]furan-2-one (S16)

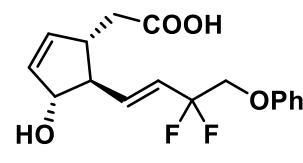

7

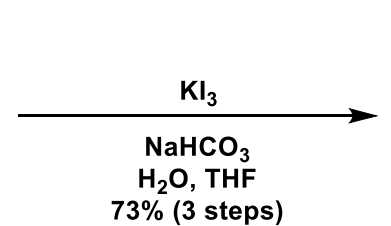

$73 \%$ (3 steps)

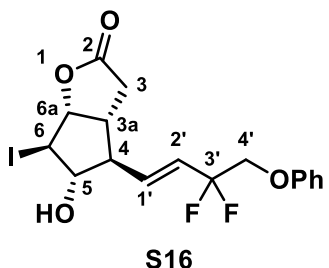

S16

Compound $\mathbf{S 1 6}$ was prepared adopting procedure by K. Yagi et al. ${ }^{[12]}$

A solution of $\mathrm{NaHCO}_{3}\left(676 \mathrm{mg}, 8.04 \mathrm{mmol}, 10.0\right.$ equiv.) in $\mathrm{H}_{2} \mathrm{O}(5.7 \mathrm{~mL})$ and solution of $\mathrm{KI}(801 \mathrm{mg}$, $4.83 \mathrm{mmol}, 6.00$ equiv.) and $\mathrm{I}_{2}\left(408 \mathrm{mg}, 1.61 \mathrm{mmol}, 2.00\right.$ equiv.) in $\mathrm{H}_{2} \mathrm{O}(5.7 \mathrm{~mL})$ were added to a solution of acid $7(289 \mathrm{mg}, 804 \mu \mathrm{mol}, 1.00$ equiv.) in THF $(3.2 \mathrm{~mL})$ at r.t.. After stirring the mixture for $24 \mathrm{~h}$ in the dark, the reaction mixture was poured onto an aq. sat. sol. of $\mathrm{Na}_{2} \mathrm{~S}_{2} \mathrm{O}_{3}(30 \mathrm{~mL})$ and was extracted with ethyl acetate $(3 \times 30 \mathrm{~mL})$. The combined organic phases were dried over $\mathrm{Na}_{2} \mathrm{SO}_{4}$ and concentrated under reduced pressure. Purification by gradient flash column chromatography (ethyl acetate/hexane $=10 \%$ to $40 \%) \mathbf{S 1 6}$ as a colorless oil ( $266 \mathrm{mg}, 73 \%$ over 3 steps $)$.

${ }^{1} \mathbf{H}$ NMR $\left(400 \mathrm{MHz},\left(\mathrm{CD}_{3}\right)_{2} \mathrm{CO}\right) \delta: 7.37-7.27\left(\mathrm{~m}, 2 \mathrm{H}, \mathrm{C}_{\text {meta }}-\mathrm{H}\right), 7.04-6.95$ (m, 3H, $\mathrm{C}_{\text {ortho }}-\mathrm{H}$ and $\left.\mathrm{C}_{\text {para }}-\mathrm{H}\right), 6.30$ (ddt, $\left.J=15.8,7.8,2.5 \mathrm{~Hz}, 1 \mathrm{H}, \mathrm{C}\left(1^{\circ}\right)-\mathrm{H}\right), 6.03$ (dtd, $\left.J=15.8,11.2,1.1 \mathrm{~Hz}, 1 \mathrm{H}, \mathrm{C}\left(2^{\circ}\right)-\mathrm{H}\right)$, $5.15-5.05(\mathrm{~m}, 1 \mathrm{H}, \mathrm{C}(6 \mathrm{a})-\mathrm{H}), 5.05-4.99(\mathrm{~m}, 1 \mathrm{H}, \mathrm{OH}), 4.34\left(\mathrm{t}, J=12.4 \mathrm{~Hz}, 2 \mathrm{H}, \mathrm{C}\left(4^{\circ}\right)-\mathrm{H}_{2}\right), 4.18-$ $4.08(\mathrm{~m}, 1 \mathrm{H}, \mathrm{C}(5)-\mathrm{H}), 4.12-4.04(\mathrm{~m}, 1 \mathrm{H}, \mathrm{C}(6)-\mathrm{H}), 3.01$ (tdd, $J=10.2,8.4,3.1 \mathrm{~Hz}, 1 \mathrm{H}, \mathrm{C}(3 \mathrm{a})-\mathrm{H})$, $2.82(\mathrm{dd}, J=18.2,10.1 \mathrm{~Hz}, 1 \mathrm{H}, \mathrm{C}(3)-\mathrm{H}), 2.54-2.46(\mathrm{~m}, 1 \mathrm{H}, \mathrm{C}(4)-\mathrm{H}), 2.42(\mathrm{dd}, J=18.2,3.1 \mathrm{~Hz}, 1 \mathrm{H}$, $\mathrm{C}(3)-\mathrm{H})$.

${ }^{13} \mathrm{C}$ NMR (101 MHz, $\left.\left(\mathrm{CD}_{3}\right)_{2} \mathrm{CO}\right)$ ): $175.8(\mathrm{C}(2)), 159.2\left(\mathrm{C}_{\mathrm{q}}\right), 137.0\left(\mathrm{t}, J=9.2 \mathrm{~Hz}, \mathrm{C}\left(1^{\circ}\right)\right), 130.4$ $\left(\mathrm{C}_{\text {meta }}\right), 126.0\left(\mathrm{t}, J=25.1 \mathrm{~Hz}, \mathrm{C}\left(2^{\circ}\right)\right), 122.5\left(\mathrm{C}_{\text {para }}\right), 119.4\left(\mathrm{t}, J=239.5 \mathrm{~Hz}, \mathrm{C}\left(3^{\circ}\right)\right), 115.7\left(\mathrm{C}_{\text {ortho }}\right), 89.9$ (C(6a)), 83.6 (C(5)), 70.0 (t, J = 33.0 Hz, C(4')), 53.9 (C(4)), 41.3 (C(3a)), 33.7 (C(6)), $33.6(\mathrm{C}(3))$.

${ }^{19}$ F NMR $\left(376 \mathrm{MHz},\left(\mathrm{CD}_{3}\right)_{2} \mathrm{CO},{ }^{1} \mathrm{H}\right.$ decoupled) $\delta$ : $-103.62(\mathrm{~d}, J=254.3 \mathrm{~Hz}),-104.34(\mathrm{~d}, J=253.3$ $\mathrm{Hz})$.

FT-IR $\left(\mathrm{CHCl}_{3}\right.$ film) cm $\mathrm{cm}^{-1}: 3422.4$ (br. w), 1760(s), 1598 (m), 1494 (m), 1247 (s), 1161 (s), 1052 (s), 973 (s), 756 (s).

HRMS (ESI) m/z: [M - H] Calcd for $\mathrm{C}_{17} \mathrm{H}_{16} \mathrm{O}_{4} \mathrm{~F}_{2} \mathrm{I}$ 449.0067; Found 449.0066.

$[\alpha]^{25}=+15.6\left(\mathrm{c}=0.5, \mathrm{CHCl}_{3}\right)$. 
(3aR,4R,5R,6aS)-4-((E)-3,3-difluoro-4-phenoxybut-1-en-1-yl)-5-hydroxyhexahydro-2Hcyclopenta[b]furan-2-one (8)
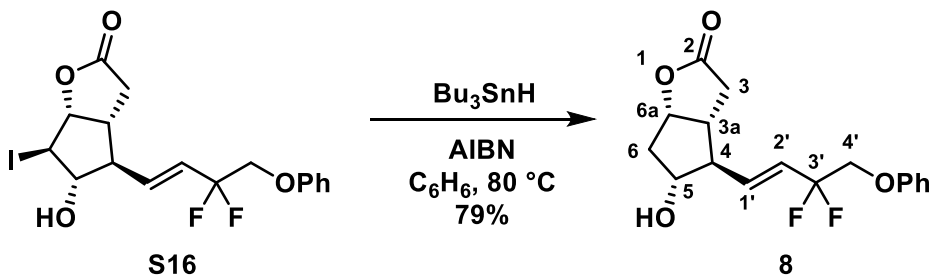

Compound $\mathbf{8}$ was prepared adopting procedure by K. Yagi et al. ${ }^{[12]}$

Tributyltinhydride (100 $\mu \mathrm{L}, 390 \mu \mathrm{mol}, 3.0$ equiv.) and azobisisobutyronitrile (AIBN) $(2.1 \mathrm{mg}$, $13 \mu \mathrm{mol}, 0.10$ equiv.) were added to solution of iodolactone $\mathbf{S 1 6}(58.2 \mathrm{mg}, 129 \mu \mathrm{mol}, 1.00$ equiv.) in benzene $(2.6 \mathrm{~mL})$. The resulting mixture was stirred at $80^{\circ} \mathrm{C}$. After $1 \mathrm{~h}$, the reaction mixture was cooled down to room temperature, solid KF $(85 \mathrm{mg})$ was added and the resulting mixture was stirred for additional $1 \mathrm{~h}$. The reaction mixture was then loaded on silica and the crude product purified by gradient flash column chromatography (ethyl acetate/hexane $=0 \%$ to $70 \%$, stationary phase $\mathrm{K}_{2} \mathrm{CO}_{3} /$ silica $\left.=1: 9\right)$. The product 8 was obtained as a colorless oil $(33.1 \mathrm{mg}, 79 \%)$.

${ }^{1}$ H NMR $\left(400 \mathrm{MHz},\left(\mathrm{CD}_{3}\right)_{2} \mathrm{CO}\right) \delta: 7.37-7.24\left(\mathrm{~m}, 2 \mathrm{H}, \mathrm{C}_{\text {meta }}-\mathrm{H}\right), 7.06-6.93\left(\mathrm{~m}, 3 \mathrm{H}, \mathrm{C}_{\text {ortho }}-\mathrm{H}\right.$ and $\left.\mathrm{C}_{\text {para }}-\mathrm{H}\right), 6.23$ (ddt, $\left.J=15.9,7.9,2.5 \mathrm{~Hz}, 1 \mathrm{H}, \mathrm{C}\left(1^{\circ}\right)-\mathrm{H}\right), 5.97$ (dtd, $\left.J=15.9,11.1,1.1 \mathrm{~Hz}, 1 \mathrm{H}, \mathrm{C}\left(2^{\circ}\right)-\mathrm{H}\right)$, $4.94(\mathrm{td}, J=7.0,3.1 \mathrm{~Hz}, 1 \mathrm{H}, \mathrm{C}(6 \mathrm{a})-\mathrm{H}), 4.33\left(\mathrm{t}, J=12.4 \mathrm{~Hz}, 2 \mathrm{H}, \mathrm{C}\left(4^{\circ}\right)-\mathrm{H}_{2}\right), 4.25(\mathrm{~d}, J=5.0 \mathrm{~Hz}, 1 \mathrm{H}$, $\mathrm{OH}), 4.09$ (qd, $J=7.1,5.1 \mathrm{~Hz}, 1 \mathrm{H}, \mathrm{C}(5)-\mathrm{H}), 2.82-2.71(\mathrm{~m}, 2 \mathrm{H}, \mathrm{C}(3 \mathrm{a})-\mathrm{H}$ and $\mathrm{C}(3)-\mathrm{H}), 2.54-2.42(\mathrm{~m}$, 2H, C(4)-H and C(6)-H), $2.42-2.30(\mathrm{~m}, 1 \mathrm{H}, \mathrm{C}(3)-\mathrm{H}), 1.84$ (ddd, $J=14.5,7.2,3.2 \mathrm{~Hz}, 1 \mathrm{H}, \mathrm{C}(6)-\mathrm{H})$.

${ }^{13}$ C NMR (101 MHz, $\left.\left(\mathrm{CD}_{3}\right)_{2} \mathrm{CO}\right)$ 8: $176.8(\mathrm{C}(2)), 159.2\left(\mathrm{C}_{\mathrm{q}}\right), 138.3\left(\mathrm{t}, J=9.1 \mathrm{~Hz}, \mathrm{C}\left(1^{\circ}\right)\right), 130.4$ $\left(\mathrm{C}_{\text {meta }}\right), 125.1$ (t, $\left.J=25.0 \mathrm{~Hz}, \mathrm{C}\left(2^{\circ}\right)\right), 122.4\left(\mathrm{C}_{\text {para }}\right), 119.5$ (t, $\left.J=239.5 \mathrm{~Hz}, \mathrm{C}\left(3^{\circ}\right)\right), 115.7\left(\mathrm{C}_{\text {ortho }}\right), 83.0$ (C(6a)), $76.9(\mathrm{C}(5)), 69.9$ (t, $\left.J=33.1 \mathrm{~Hz}, \mathrm{C}\left(4^{\circ}\right)\right), 56.5(\mathrm{C}(4)), 42.8(\mathrm{C}(3 \mathrm{a})), 41.2(\mathrm{C}(6)), 34.6(\mathrm{C}(3))$.

${ }^{19}$ F NMR (377 MHz, $\left(\mathrm{CD}_{3}\right)_{2} \mathrm{CO}$, decoupled) $\delta:-103.41(\mathrm{~d}, J=253.7 \mathrm{~Hz}),-104.17(\mathrm{~d}, J=253.0 \mathrm{~Hz})$.

FT-IR $\left(\mathrm{CHCl}_{3}\right.$ film) cm $\mathrm{cm}^{-1}: 3431$ (br. w), 2932 (w), 1760 (s), 1598 (m), 1494 (m), 1247 (s), 1161 (s), 1079 (s), 1025 (s), 973 (s), 973 (m), 756 (s), 692 (s).

HRMS (ESI) m/z: [M + Na $]^{+}$Calcd for $\mathrm{C}_{17} \mathrm{H}_{18} \mathrm{O}_{4} \mathrm{~F}_{2} \mathrm{Na}$ 347.1065; Found 347.1065.

$[\alpha]^{25}{ }^{\mathbf{D}}=-1.9\left(\mathrm{c}=0.5, \mathrm{CHCl}_{3}\right)$. 


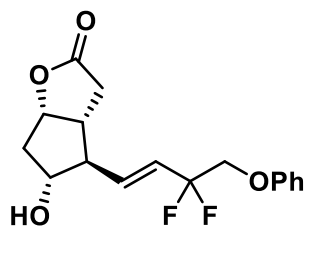

8

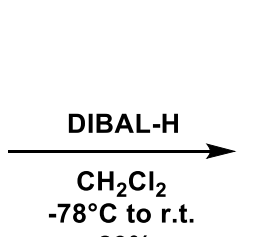

$89 \%$

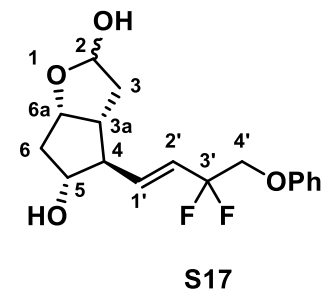

S17

Compound $\mathbf{S 1 6}$ was prepared adopting procedure by S. Prevost et al. ${ }^{[13]}$

A solution of diisobutylaluminium hydride (DIBAL-H) $\left(1 \mathrm{M}\right.$ in $\mathrm{CH}_{2} \mathrm{Cl}_{2}, 580 \mu \mathrm{L}, 580 \mu \mathrm{mol}, 2.2$ equiv.) was added to a solution of $8\left(85.0 \mathrm{mg}, 262 \mu \mathrm{mol}, 1.00\right.$ equiv.) in $\mathrm{CH}_{2} \mathrm{Cl}_{2}(5.4 \mathrm{~mL})$ at $-78{ }^{\circ} \mathrm{C}$. After stirring for $1 \mathrm{~h}$, the reaction mixture was allowed to warm to r.t., quenched with an aq. sat. sol. of potassium sodium tartrate $(10 \mathrm{~mL})$, and was stirred for additional $1 \mathrm{~h}$. The reaction mixture was then extracted with $\mathrm{CH}_{2} \mathrm{Cl}_{2}(4 \times 15 \mathrm{~mL})$, the combined organic phases were washed with brine (10 $\mathrm{mL}$ ), dried over $\mathrm{Na}_{2} \mathrm{SO}_{4}$, and concentrated under reduced pressure. Purification by flash column chromatography (ethyl acetate/hexane $=70 \%$ ) afforded $\mathbf{S 1 6}$ as colorless oil $(76.4 \mathrm{mg}, 89 \%)$ as an inseparable mixture of two diastereomers.

${ }^{1} \mathbf{H}$ NMR $\left(500 \mathrm{MHz}, \mathrm{CDCl}_{3}\right) \delta: 7.35-7.27\left(\mathrm{~m}, 4 \mathrm{H}, \mathrm{C}_{\text {meta }}-\mathrm{H}\right.$ and $\left.\mathrm{C}^{{ }^{6}}{ }_{\text {meta }}-\mathrm{H}\right), 7.01$ (ddt, $J=8.4,7.2,1.2$ $\mathrm{Hz}, 2 \mathrm{H}, \mathrm{C}_{\mathrm{para}}-\mathrm{H}$ and $\left.\mathrm{C}^{\text {para }}-\mathrm{H}\right), 6.95-6.88\left(\mathrm{~m}, 4 \mathrm{H}, \mathrm{C}_{\text {ortho }}-\mathrm{H}\right.$ and $\left.\mathrm{C}_{\text {ortho }}-\mathrm{H}\right), 6.15-6.04\left(\mathrm{~m}, \mathrm{C}\left(1^{\circ}\right)-\mathrm{H}\right.$ and $\left.\mathrm{C}^{\prime}\left(1^{\circ}\right)-\mathrm{H}\right), 5.88-5.74\left(\mathrm{~m}, 2 \mathrm{H}, \mathrm{C}\left(2^{\circ}\right)-\mathrm{H}\right.$ and $\left.\mathrm{C}^{\circ}\left(2^{\circ}\right)-\mathrm{H}\right), 5.67(\mathrm{~d}, J=5.0 \mathrm{~Hz}, 1 \mathrm{H}, \mathrm{C}(2)-\mathrm{H}), 5.58(\mathrm{~d}, J=$ $\left.5.5 \mathrm{~Hz}, 1 \mathrm{H}, \mathrm{C}^{\prime}(2)-\mathrm{H}\right), 4.69$ (dt, $\left.J=6.7,3.3 \mathrm{~Hz}, 1 \mathrm{H}, \mathrm{C}(6 \mathrm{a})-\mathrm{H}\right), 4.64$ (td, $\left.J=7.1,3.4 \mathrm{~Hz}, 1 \mathrm{H}, \mathrm{C}^{\prime}(6 \mathrm{a})-\mathrm{H}\right)$, $4.19\left(\mathrm{t}, J=11.4 \mathrm{~Hz}, 4 \mathrm{H}, \mathrm{C}\left(4^{\circ}\right)-\mathrm{H}_{2}\right.$ and $\left.\mathrm{C}^{\prime}\left(4^{\circ}\right)-\mathrm{H}_{2}\right), 4.01-3.93\left(\mathrm{~m}, 2 \mathrm{H}, \mathrm{C}(5)-\mathrm{H}\right.$ and $\left.\mathrm{C}^{\prime}(5)-\mathrm{H}\right), 2.89$ (q, $J$ $\left.=6.9 \mathrm{~Hz}, 1 \mathrm{H}, \mathrm{C}^{`}(4)-\mathrm{H}\right), 2.80(\mathrm{~s}, 1 \mathrm{H}, \mathrm{C}(2)-\mathrm{OH}), 2.56(\mathrm{dtd}, J=8.9,7.2,4.5 \mathrm{~Hz}, 1 \mathrm{H}, \mathrm{C}(3 \mathrm{a})-\mathrm{H}), 2.49$ (dtd, $\left.J=8.5,7.1,1.3 \mathrm{~Hz}, 1 \mathrm{H}, \mathrm{C}^{\prime}(3 \mathrm{a})-\mathrm{H}\right), 2.40-2.31\left(\mathrm{~m}, 3 \mathrm{H}, \mathrm{C}(4)-\mathrm{H}, \mathrm{C}(6)-\mathrm{H}\right.$ and $\left.\mathrm{C}^{\prime}(6)-\mathrm{H}\right), 2.24$ (ddd, $J=$ $\left.13.8,9.7,5.6 \mathrm{~Hz}, 1 \mathrm{H}, \mathrm{C}^{\circ}(3)-\mathrm{H}\right), 2.14$ (ddd, $\left.J=13.6,9.0,1.6 \mathrm{~Hz}, 1 \mathrm{H}, \mathrm{C}(3)-\mathrm{H}\right), 2.09$ (ddd, $J=14.2,6.1$, $\left.3.5 \mathrm{~Hz}, 1 \mathrm{H}, \mathrm{C}^{\prime}(6)-\mathrm{H}\right), 2.06-1.97\left(\mathrm{~m}, 2 \mathrm{H}, \mathrm{C}(3)-\mathrm{H}\right.$ and $\left.\mathrm{C}^{\prime}(3)-\mathrm{H}\right), 1.85(\mathrm{ddd}, J=14.2,6.8,3.1 \mathrm{~Hz}, 1 \mathrm{H}$, $\mathrm{C}(6)-\mathrm{H})$.

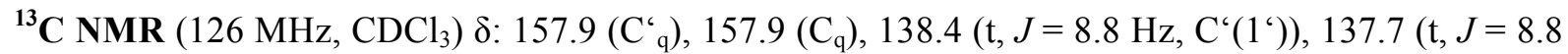
$\left.\mathrm{Hz}, \mathrm{C}\left(1^{\circ}\right)\right), 129.79\left(\mathrm{C}_{\text {meta }}\right), 129.77\left(\mathrm{C}^{\circ}{ }_{\text {meta }}\right), 124.0\left(\mathrm{t}, J=24.9 \mathrm{~Hz}, \mathrm{C}\left(2^{\circ}\right)\right), 123.5\left(\mathrm{t}, J=24.8 \mathrm{~Hz}, \mathrm{C}^{\prime}\left(2^{\circ}\right)\right)$, $122.01\left(\mathrm{C}_{\text {para }}\right), 121.98\left(\mathrm{C}^{\prime}\right.$ para $), 118.26$ (t, $\left.J=240.4 \mathrm{~Hz}, \mathrm{C}^{`}\left(3^{\circ}\right)\right), 118.20$ (t, $\left.J=240.5 \mathrm{~Hz}, \mathrm{C}^{\circ}\left(3^{\circ}\right)\right), 114.8$ $\left(\mathrm{C}_{\text {ortho }}\right.$ and $\left.\mathrm{C}^{6}{ }_{\text {ortho }}\right), 101.1\left(\mathrm{C}^{\prime}(2)\right), 100.0(\mathrm{C}(2)), 84.5\left(\mathrm{C}^{\prime}(6 \mathrm{a})\right), 81.3(\mathrm{C}(6 \mathrm{a})), 78.4\left(\mathrm{C}(5)\right.$ and $\left.\mathrm{C}^{`}(5)\right), 69.4$ $\left(\mathrm{t}, J=35.3 \mathrm{~Hz}, \mathrm{C}\left(4^{\circ}\right)\right.$ and $\left.\mathrm{C} ;\left(4^{\circ}\right)\right), 57.2(\mathrm{C}(4)), 57.0\left(\mathrm{C}^{\circ}(4)\right), 46.6\left(\mathrm{C}^{`}(3 \mathrm{a})\right), 45.6(\mathrm{C}(3 \mathrm{a})), 42.8\left(\mathrm{C}^{\prime}(6)\right)$, $39.8(\mathrm{C}(6)$ or $\mathrm{C}(3)), 39.7(\mathrm{C}(6)$ or $\mathrm{C}(3)), 39.3\left(\mathrm{C}^{\prime}(3)\right)$.

${ }^{19}$ F NMR $\left(471 \mathrm{MHz}, \mathrm{CDCl}_{3}\right) \delta$ : $-102.44(\mathrm{~d}, J=256.8 \mathrm{~Hz}),-102.82(\mathrm{~d}, J=257.8 \mathrm{~Hz}),-102.87(\mathrm{~d}, J=$ $256.5 \mathrm{~Hz}),-103.28(\mathrm{~d}, J=256.5 \mathrm{~Hz})$.

FT-IR $\left(\mathrm{CHCl}_{3}\right.$ film) $\mathrm{cm}^{-1}: 3372$ (br. w), 1496 (m), 1294 (m), 1060 (m).

HRMS (ESI) m/z: [M + Na] $]^{+}$Calcd for $\mathrm{C}_{17} \mathrm{H}_{20} \mathrm{O}_{4} \mathrm{~F}_{2} \mathrm{Na}$ 349.1222; Found 349.1223.

$[\alpha]^{25}{ }^{25}=-10.4\left(\mathrm{c}=0.5, \mathrm{CHCl}_{3}\right)$. 


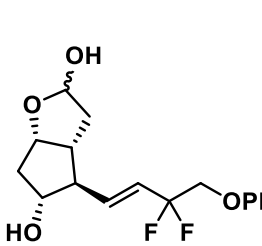

S17

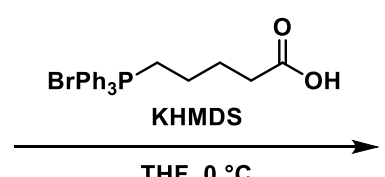

THF, $0^{\circ} \mathrm{C}$

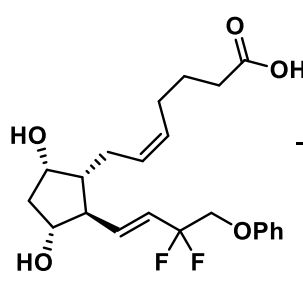

S18

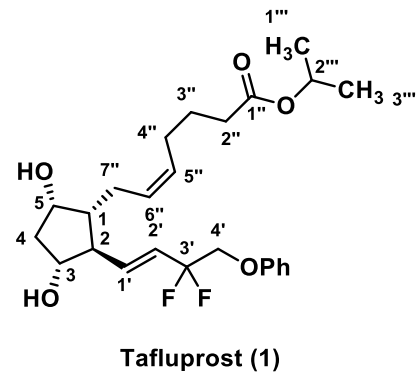

Compound 1 was prepared adopting procedure by Y. Zhao et al. ${ }^{[14]}$

A solution of potassium bis(trimethylsilyl)amide (KHMDS) $(0.5 \mathrm{M}$ in toluene, $490 \mu \mathrm{L}, 250 \mu \mathrm{mol}, 8.0$ equiv.) was added to a suspension of (4-Carboxybutyl)triphenylphosphonium bromide $(54.2 \mathrm{mg}, 123$ $\mu$ mol, 4.00 equiv.) in THF $(350 \mu \mathrm{L})$ at $0{ }^{\circ} \mathrm{C}$ (intensively orange solution). After stirring for $15 \mathrm{~min}$, a solution of hemiacetal $\mathbf{S 1 7}(10 \mathrm{mg}, 30.6 \mu \mathrm{mol}, 1.00$ equiv.) in THF $(300 \mu \mathrm{L})$ was added and the resulting solution was stirred for $2 \mathrm{~h}$ at $0{ }^{\circ} \mathrm{C} . \mathrm{H}_{2} \mathrm{O}(0.5 \mathrm{~mL})$ was added, the reaction mixture was acidified with aq. $1 \mathrm{M} \mathrm{HCl}$, and extracted with ethyl acetate $(3 \times 10 \mathrm{~mL})$. The combined organic phases were washed with brine $(10 \mathrm{~mL})$, dried over $\mathrm{Na}_{2} \mathrm{SO}_{4}$, and concentrated under reduced pressure. The crude product $\mathbf{S 1 8}$ was passed through plug of silica (ethyl acetate/acetic acid $=99 \%$ ) and used directly in the next step without any further purification.

Crude S18 was dissolved in acetone ( $400 \mu \mathrm{L})$ and DBU ( $28 \mu \mathrm{L}, 180 \mu \mathrm{mol}, 6.0$ equiv.). 2-iodopropane (18 $\mu \mathrm{L}, 180 \mu \mathrm{mol}, 6.0$ equiv.) was added to the mixture and the resulting solution was stirred for $22 \mathrm{~h}$ at r.t. The reaction mixture was then directly loaded on silica and purified by gradient flash column chromatography (ethyl acetate/hexane $=30 \%$ to $70 \%$ ). Tafluprost (1) was obtained as a colorless oil (9.0 mg, 65\%, 90\% brsm)

The spectroscopic data are in agreement with the literature. ${ }^{[15,16,17]}$

${ }^{1} \mathbf{H}$ NMR $\left(500 \mathrm{MHz}, \mathrm{CDCl}_{3}\right) \delta: 7.34-7.26\left(\mathrm{~m}, 2 \mathrm{H}, \mathrm{C}_{\text {meta }}-\mathrm{H}\right), 7.00\left(\mathrm{tt}, J=7.3,1.1 \mathrm{~Hz}, 1 \mathrm{H}, \mathrm{C}_{\mathrm{para}}-\mathrm{H}\right)$, $6.95-6.88\left(\mathrm{~m}, 2 \mathrm{H}, \mathrm{C}_{\text {ortho }}-\mathrm{H}\right), 6.10\left(\mathrm{ddt}, J=15.8,9.1,2.5 \mathrm{~Hz}, 1 \mathrm{H}, \mathrm{C}\left(1^{\circ}\right)-\mathrm{H}\right), 5.80(\mathrm{dt}, J=15.7,11.3$ $\left.\mathrm{Hz}, 1 \mathrm{H}, \mathrm{C}\left(2^{\circ}\right)-\mathrm{H}\right), 5.44-5.32\left(\mathrm{~m}, 2 \mathrm{H}, \mathrm{C}\left(6^{\circ}\right)-\mathrm{H}\right.$ and $\left.\mathrm{C}\left(5^{\circ}\right)-\mathrm{H}\right), 4.99\left(\mathrm{~h}, J=6.3 \mathrm{~Hz}, 1 \mathrm{H}, \mathrm{C}\left(2^{\prime \prime}\right)-\mathrm{H}\right)$, $4.25-4.16\left(\mathrm{~m}, 3 \mathrm{H}, \mathrm{C}\left(4^{\circ}\right)-\mathrm{H}_{2}\right.$ and $\left.\mathrm{C}(5)-\mathrm{H}\right), 4.06-3.99(\mathrm{~m}, 1 \mathrm{H}, \mathrm{C}(3)-\mathrm{H}), 2.47(\mathrm{td}, J=9.7,4.0 \mathrm{~Hz}, 1 \mathrm{H}$, $\mathrm{C}(2)-\mathrm{H}), 2.42(\mathrm{~d}, J=7.2 \mathrm{~Hz}, 1 \mathrm{H}, \mathrm{C}(3)-\mathrm{OH}), 2.38-2.29\left(\mathrm{~m}, 1 \mathrm{H}, \mathrm{C}\left(7^{\circ} \cdot \mathrm{c}\right)-\mathrm{H}\right), 2.26(\mathrm{td}, J=7.2,2.4 \mathrm{~Hz}$, $\left.2 \mathrm{H}, \mathrm{C}\left(2^{\prime c}\right)-\mathrm{H}_{2}\right), 2.19-1.96\left(\mathrm{~m}, 4 \mathrm{H}, \mathrm{C}(4)-\mathrm{H}, \mathrm{C}\left(7^{\prime c}\right)-\mathrm{H}\right.$ and $\left.\mathrm{C}\left(4^{\prime c}\right)-\mathrm{H}_{2}\right), 1.85$ (d, $J=14.6 \mathrm{~Hz}, 1 \mathrm{H}, \mathrm{C}(4)-$ $\mathrm{H}), 1.73-1.63\left(\mathrm{~m}, 2 \mathrm{H}, \mathrm{C}\left(3^{\prime \prime}\right)-\mathrm{H}_{2}\right), 1.61(\mathrm{~m}, 1 \mathrm{H}, \mathrm{C}(1)-\mathrm{H}), 1.22\left(\mathrm{~d}, J=6.3 \mathrm{~Hz}, 6 \mathrm{H}, \mathrm{C}\left(1^{\prime \prime c}\right)-\mathrm{H}_{3}\right.$ and $\left.\mathrm{C}\left(3^{، “}\right)-\mathrm{H}_{3}\right)$. (One exchanging proton of $\mathrm{OH}$ not visible.)

${ }^{13}$ C NMR (126 MHz, $\left.\mathrm{CDCl}_{3}\right) \delta: 173.6\left(\mathrm{C}\left(1^{\prime \prime}\right)\right), 158.1\left(\mathrm{C}_{\mathrm{q}}\right), 138.8\left(\mathrm{t}, J=8.8 \mathrm{~Hz}, \mathrm{C}\left(1^{\circ}\right)\right), 130.3\left(\mathrm{C}\left(6^{\circ}\right.\right.$ “ $\left.)\right)$, $129.8\left(\mathrm{C}_{\text {meta }}\right), 128.8\left(\mathrm{C}\left(5^{\prime \prime}\right)\right), 123.7\left(\mathrm{t}, J=24.9 \mathrm{~Hz}, \mathrm{C}\left(2^{\circ}\right)\right), 122.0\left(\mathrm{C}_{\text {para }}\right), 118.3\left(\mathrm{t}, J=240.3 \mathrm{~Hz}, \mathrm{C}\left(3^{\circ}\right)\right)$, 114.9 ( $\left.\mathrm{C}_{\text {ortho }}\right), 78.2(\mathrm{C}(3)), 73.5(\mathrm{C}(5)), 69.6\left(\mathrm{t}, J=35.0 \mathrm{~Hz}, \mathrm{C}\left(4^{\circ}\right)\right), 67.8\left(\mathrm{C}\left(2^{\text {‘ }}{ }^{\prime c}\right)\right), 56.0(\mathrm{C}(2)), 50.7$

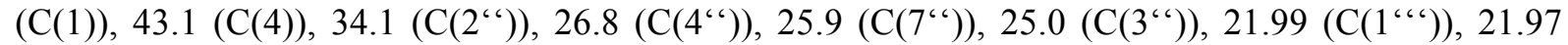
$\left(\mathrm{C}\left(33^{\prime \prime}\right)\right)$.

${ }^{19}$ F NMR $\left(470 \mathrm{MHz}, \mathrm{CDCl}_{3},{ }^{1} \mathrm{H}\right.$ decoupled $) \delta:-102.46(\mathrm{~d}, J=255.7 \mathrm{~Hz}),-103.21(\mathrm{~d}, J=255.6 \mathrm{~Hz})$.

FT-IR (CHCl ${ }_{3}$ film) cm $\mathrm{cm}^{-1}: 3395$ (br. w), 2933 (w), 1725 (s), 1599 (m), 1497(s), 1249 (s), 1108 (s), 755 (s).

HRMS (ESI) m/z: [M + Na $]^{+}$Calcd for $\mathrm{C}_{25} \mathrm{H}_{34} \mathrm{O}_{5} \mathrm{~F}_{2} \mathrm{Na}$ 475.2267; Found 475.2264.

$[\boldsymbol{\alpha}]^{25}{ }_{\mathbf{D}}=+22.4\left(\mathrm{c}=0.5, \mathrm{CHCl}_{3}\right)$, lit $[\boldsymbol{\alpha}]^{25}{ }_{\mathbf{D}}=+21.6\left(\mathrm{c}=1.0, \mathrm{CHCl}_{3}\right){ }^{[17]}$ 
TABLE S1: Comparison of ${ }^{1} \mathrm{H}$ and ${ }^{13} \mathrm{C}$ NMR data of the synthetized Tafluprost with literature.

\begin{tabular}{|c|c|c|c|c|}
\hline $\begin{array}{c}\text { Reported }{ }^{1} \mathrm{H} \\
\left.{\text { NMR }\left(600 \mathrm{MHz}^{2}\right.} \mathrm{CDCl}_{3}\right)^{[16]}\end{array}$ & $\begin{array}{c}\text { Reported }{ }^{1} \mathrm{H} \text { NMR } \\
\text { (unknown frequency, } \\
\left.\mathrm{CDCl}_{3}\right)^{[15]}\end{array}$ & $\begin{array}{c}\text { Measured }^{1} \mathrm{H} \\
\left.{\text { NMR }\left(500 \mathrm{MHz}^{2}\right.} \text { CDCl }_{3}\right)\end{array}$ & $\begin{array}{c}\text { Reported }{ }^{13} \mathrm{C} \\
\text { NMR }(150 \mathrm{MHz} \\
\left.\mathrm{CDCl}_{3}\right)^{[16]}\end{array}$ & $\begin{array}{c}\text { Measured }{ }^{13} \mathrm{C} \\
\text { NMR }(126 \mathrm{MHz} \\
\left.\mathrm{CDCl}_{3}\right)\end{array}$ \\
\hline $1.22(\mathrm{~d}, 6 \mathrm{H})$ & $\begin{array}{l}1.22(\mathrm{~d}, J=6.2 \mathrm{~Hz} \\
3 \mathrm{H}) \text { and } 1.22(\mathrm{~d}, J= \\
6.2 \mathrm{~Hz}, 3 \mathrm{H})\end{array}$ & $\begin{array}{l}1.22(\mathrm{~d}, J=6.3 \mathrm{~Hz}, \\
6 \mathrm{H})\end{array}$ & $21.8(2 \mathrm{~s})$ & 21.99 and 21.97 \\
\hline $1.61(\mathrm{~m}, 1 \mathrm{H})$ & $1.58-1.63(\mathrm{~m}, 1 \mathrm{H})$ & $1.61(\mathrm{~m}, 1 \mathrm{H})$ & 24.8 & 25.0 \\
\hline $1.67(\mathrm{~m}, 2 \mathrm{H})$ & $1.63-1.69(\mathrm{~m}, 2 \mathrm{H})$ & $\begin{array}{l}1.63-1.73(\mathrm{~m}, \\
2 \mathrm{H})\end{array}$ & 25.7 & 25.9 \\
\hline $1.85(\mathrm{~m}, 1 \mathrm{H})$ & $\begin{array}{l}1.84(\mathrm{~d}, J=14,7 \mathrm{~Hz}, \\
1 \mathrm{H})\end{array}$ & $\begin{array}{l}1.85(\mathrm{~d}, J=14.6 \\
\mathrm{Hz}, 1 \mathrm{H})\end{array}$ & 26.6 & 26.8 \\
\hline $2.06(\mathrm{~m}, 1 \mathrm{H})$ & $2.02-2.08(\mathrm{~m}, 1 \mathrm{H})$ & $\begin{array}{l}1.96-2.19(\mathrm{~m}, \\
4 \mathrm{H})\end{array}$ & 34.0 & 34.1 \\
\hline \multirow[t]{4}{*}{$2.09-2.16(\mathrm{~m}, 3 \mathrm{H})$} & $2.10-2.16(\mathrm{~m}, 3 \mathrm{H})$ & & 42.9 & 43.1 \\
\hline & $2.25(\mathrm{t}, J=7.3 \mathrm{~Hz}, 1 \mathrm{H})$ & $\begin{array}{l}2.26(\mathrm{td}, J=7.2, \\
2.4 \mathrm{~Hz}, 2 \mathrm{H})\end{array}$ & 50.5 & 50.7 \\
\hline & $2.26(\mathrm{t}, J=7.1 \mathrm{~Hz}, 1 \mathrm{H})$ & & 55.7 & 56.0 \\
\hline & $2.30-2.35(\mathrm{~m}, 1 \mathrm{H})$ & $\begin{array}{l}2.29-2.38 \quad(\mathrm{~m}, \\
1 \mathrm{H})\end{array}$ & 67.7 & 67.8 \\
\hline a & $2.46-2.49(\mathrm{~m}, 2 \mathrm{H})$ & $\begin{array}{l}2.42(\mathrm{~d}, J=7.2 \mathrm{~Hz} \\
1 \mathrm{H})\end{array}$ & $69.5(J=34.9 \mathrm{~Hz})$ & $\begin{array}{l}69.6(\mathrm{t}, J=35.0 \\
\mathrm{Hz})\end{array}$ \\
\hline $2.47(\mathrm{~m}, 1 \mathrm{H})$ & & $\begin{array}{l}2.47(\mathrm{td}, J=9.7 \\
4.0 \mathrm{~Hz}, 1 \mathrm{H})\end{array}$ & 73.3 & 73.5 \\
\hline $\mathrm{b}$ & $2.61-2.63(\mathrm{~m}, 1 \mathrm{H})$ & & 77.9 & 78.2 \\
\hline $4.02(\mathrm{~m}, 1 \mathrm{H})$ & $4.02-4.03(\mathrm{~m}, 1 \mathrm{H})$ & $\begin{array}{l}3.99-4.06(\mathrm{~m}, \\
1 \mathrm{H})\end{array}$ & 114.8 & 114.9 \\
\hline $\begin{array}{l}4.17-4.22(\mathrm{~m}, \\
2 \mathrm{H})^{\mathrm{c}}\end{array}$ & $4.18-4.21(\mathrm{~m}, 3 \mathrm{H})$ & $\begin{array}{l}4.16-4.25(\mathrm{~m}, \\
3 \mathrm{H})\end{array}$ & $\begin{array}{l}118.2(J=240.0 \\
\mathrm{Hz})\end{array}$ & $\begin{array}{l}118.3(\mathrm{t}, J=240.3 \\
\mathrm{Hz})\end{array}$ \\
\hline $\begin{array}{l}5.00(\mathrm{sept}, J=6.30 \\
\mathrm{Hz}, 1 \mathrm{H})\end{array}$ & $\begin{array}{l}5.00 \text { (heptet, } J=6.2 \\
\mathrm{~Hz}, 1 \mathrm{H})\end{array}$ & $\begin{array}{l}4.99(\mathrm{~h}, J=6.3 \mathrm{~Hz}, \\
1 \mathrm{H})\end{array}$ & 121.8 & 122.0 \\
\hline $5.33-5.42(\mathrm{~m}, 2 \mathrm{H})$ & $5.35-5.42(\mathrm{~m}, 2 \mathrm{H})$ & $\begin{array}{l}5.32-5.44(\mathrm{~m}, \\
2 \mathrm{H})\end{array}$ & $123.6(J=25.0 \mathrm{~Hz})$ & $\begin{array}{l}123.7(\mathrm{t}, J=24.9 \\
\mathrm{Hz})\end{array}$ \\
\hline $5.80(\mathrm{~m}, 1 \mathrm{H})$ & $\begin{array}{l}5.80(\mathrm{dt}, J=15.8,11.2 \\
\mathrm{Hz}, 1 \mathrm{H})\end{array}$ & $\begin{array}{l}5.80(\mathrm{dt}, J=15.7, \\
11.3 \mathrm{~Hz}, 1 \mathrm{H})\end{array}$ & 128.6 & 128.8 \\
\hline $6.10(\mathrm{~m}, 1 \mathrm{H})$ & $\begin{array}{l}6.10(\mathrm{dd}, J=15.8,8.8 \\
\mathrm{Hz}, 1 \mathrm{H})\end{array}$ & $\begin{array}{l}6.10(\mathrm{ddt}, J=15.8, \\
9.1,2.5 \mathrm{~Hz}, 1 \mathrm{H})\end{array}$ & 129.6 & 129.8 \\
\hline $6.92(\mathrm{~m}, 2 \mathrm{H})$ & $\begin{array}{l}6.91(\mathrm{~d}, J=8.8 \mathrm{~Hz} \\
2 \mathrm{H})\end{array}$ & $\begin{array}{l}6.88-6.95(\mathrm{~m}, \\
2 \mathrm{H})\end{array}$ & 130.1 & 130.3 \\
\hline $7.00(\mathrm{~m}, 1 \mathrm{H})$ & $7.00(\mathrm{t}, J=7.3 \mathrm{~Hz}, 1 \mathrm{H})$ & $\begin{array}{l}7.00(\mathrm{tt}, J=7.3, \\
1.1 \mathrm{~Hz}, 1 \mathrm{H})\end{array}$ & $138.6(J=8.8 \mathrm{~Hz})$ & $\begin{array}{l}138.8(\mathrm{t}, J=8.8 \\
\mathrm{Hz})\end{array}$ \\
\hline \multirow[t]{2}{*}{$7.30(\mathrm{~m}, 2 \mathrm{H})$} & $\begin{array}{l}7.30(\mathrm{dd}, J=8.8,7.3 \\
\mathrm{Hz}, 2 \mathrm{H})\end{array}$ & $\begin{array}{l}7.26-7.34(\mathrm{~m}, \\
2 \mathrm{H})\end{array}$ & 157.9 & 158.1 \\
\hline & & & 173.5 & 173.6 \\
\hline
\end{tabular}

${ }^{a}$ Exchanging $\mathrm{OH}$ visible ${ }^{b}$ Exchanging $\mathrm{OH}$ not visible. ${ }^{c}$ One proton not reported.

TABLE S2: Comparison of ${ }^{19}$ F NMR data of the synthetized Tafluprost with literature.

\begin{tabular}{|c|l|c|}
\hline $\begin{array}{c}\text { Reported }{ }^{19} \mathbf{F} \text { NMR (470 MHz, } \\
\left.\mathbf{C D C l}_{\mathbf{3}}\right)^{[16]}\end{array}$ & $\begin{array}{c}\text { Reported }{ }^{19} \mathbf{F} \text { NMR (unknown } \\
\left.\text { frequency, } \mathbf{C D C l}_{\mathbf{3}}\right)^{[15]}\end{array}$ & $\begin{array}{c}\text { Measured } \\
{ }^{19} \mathbf{F} \text { NMR (470 } \\
\left.\text { MHz, } \mathbf{C D C l}_{\mathbf{3}}\right)\end{array}$ \\
\hline$-102.6\left({ }^{2} J_{\mathrm{F}-\mathrm{F}}=255.8 \mathrm{~Hz}\right)$ & $-102.8\left({ }^{2} J_{\mathrm{F}-\mathrm{F}}=255.6 \mathrm{~Hz}\right)$ & $-102.5(\mathrm{~d}, J=255.7 \mathrm{~Hz})$ \\
\hline$-103.1\left({ }^{2} J_{\mathrm{F}-\mathrm{F}}=255.8 \mathrm{~Hz}\right)$ & $-103.6\left({ }^{2} J_{\mathrm{F}-\mathrm{F}}=255.6 \mathrm{~Hz}\right)$ & $-103.2(\mathrm{~d}, J=255.6 \mathrm{~Hz})$ \\
\hline
\end{tabular}




\section{References}

[1] R. Uson, L. A. Oro, J. A. Cabeza, H. E. Bryndza, M. P. Stepro, in Inorg. Synth., John Wiley \& Sons, Ltd, 2007, pp. 126-130.

[2] M. E. Jung, J. A. Berliner, D. Angst, D. Yue, L. Koroniak, A. D. Watson, R. Li, Org. Lett. 2005, 7, 3933-3935.

[3] F. W. Goetzke, M. Mortimore, S. P. Fletcher, Angew. Chem. Int. Ed. 2019, 58, 12128-12132.

[4] B. M. Trost, M. T. Sorum, Org. Process Res. Dev. 2003, 7, 432-435.

[5] D. R. Dragoli, L. A. Thompson, J. O’Brie, J. A. Ellman, J. Comb. Chem. 1999, 1, 534-539.

[6] L. Preti, O. A. Attanasi, E. Caselli, G. Favi, C. Ori, P. Davoli, F. Felluga, F. Prati, Eur. J. Org. Chem. 2010, 2010, 4312-4320.

[7] W.-W. Qiu, K. Surendra, L. Yin, E. J. Corey, Org. Lett. 2011, 13, 5893-5895.

[8] O. W. Gooding, C. C. Beard, G. F. Cooper, D. Y. Jackson, J. Org. Chem. 1993, 58, 3681-3686.

[9] J.-F. Syu, Y.-T. Wang, K.-C. Liu, P.-Y. Wu, J. P. Henschke, H.-L. Wu, J. Org. Chem. 2016, 81, 10832-10844.

[10] H. L. Sebahar, K. Yoshida, L. S. Hegedus, J. Org. Chem. 2002, 67, 3788-3795.

[11] D. Lafrance, P. Bowles, K. Leeman, R. Rafka, Org. Lett. 2011, 13, 2322-2325.

[12] K. Yagi, H. Nonaka, H. P. Acharya, K. Furukawa, T. Ainai, Y. Kobayashi, Tetrahedron 2006, 62, 4933-4940.

[13] S. Prévost, K. Thai, N. Schützenmeister, G. Coulthard, W. Erb, V. K. Aggarwal, Org. Lett. 2015, $17,504-507$.

[14] Y. Zhao, Y. Li, U. Kotipalli, S. C. Duncan, H. LV, K. LI, Salts of Prostaglandin Analog Intermediates, 2016, WO2016090461A1.

[15] Y. Matsumura, N. Mori, T. Nakano, H. Sasakura, T. Matsugi, H. Hara, Y. Morizawa, Tetrahedron Lett. 2004, 45, 1527-1529.

[16] M. Krupa, M. Chodyński, A. Ostaszewska, P. Cmoch, I. Dams, Molecules 2017, 22, 217

[17] Wu, P.; Wu, H.; Wen, W. Metal-Catalyzed Asymmetric 1,4-Conjugate Addition of Vinylboron Compounds to 2-Substituted-4-Oxy-Cyclopent-2-en-1-ones Yielding Prostaglandins and Prostaglandin Analogs, 2016, WO2016/005943A1. 


\section{NMR spectra}

通

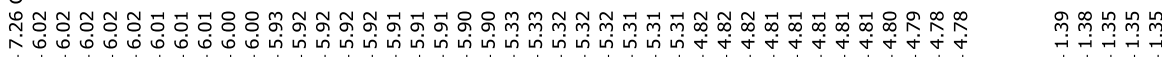

$\underbrace{\mathrm{Cl}}_{\cdot{ }^{\prime \prime}} \mathrm{C}_{\mathrm{CH}_{3}}^{\mathrm{CH}_{3}}$

(士)-2
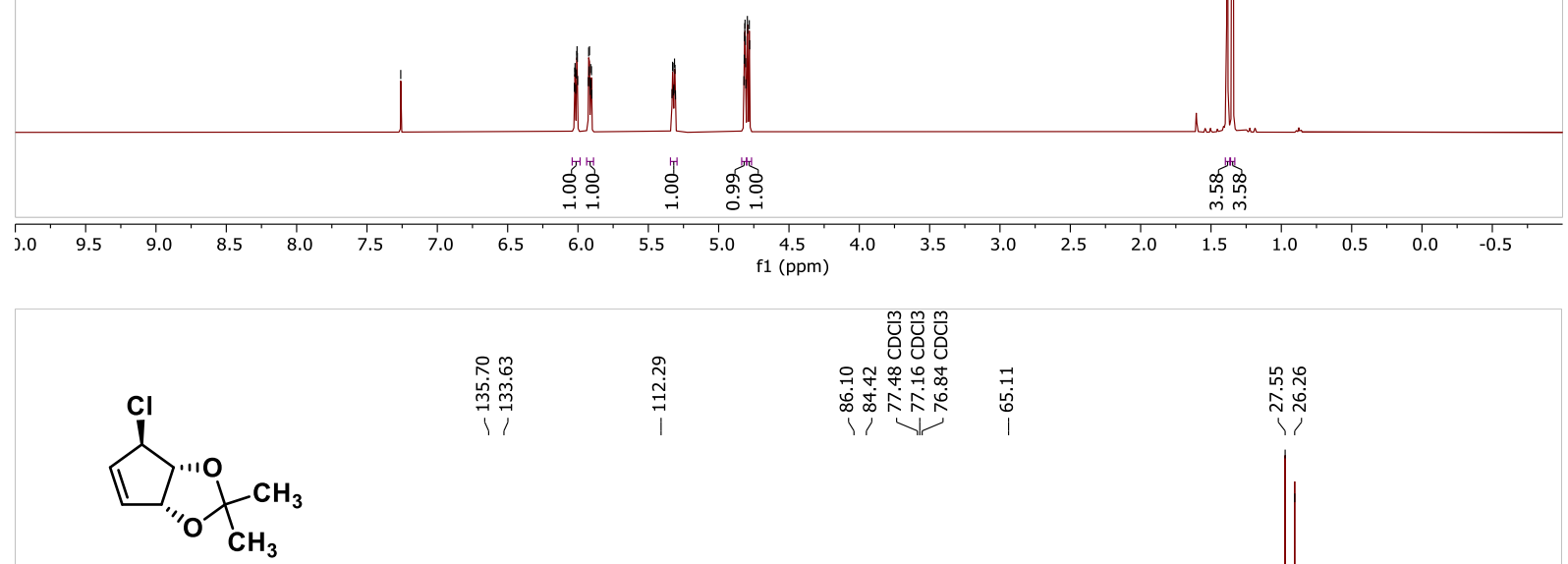

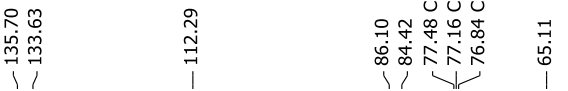

(士)-2

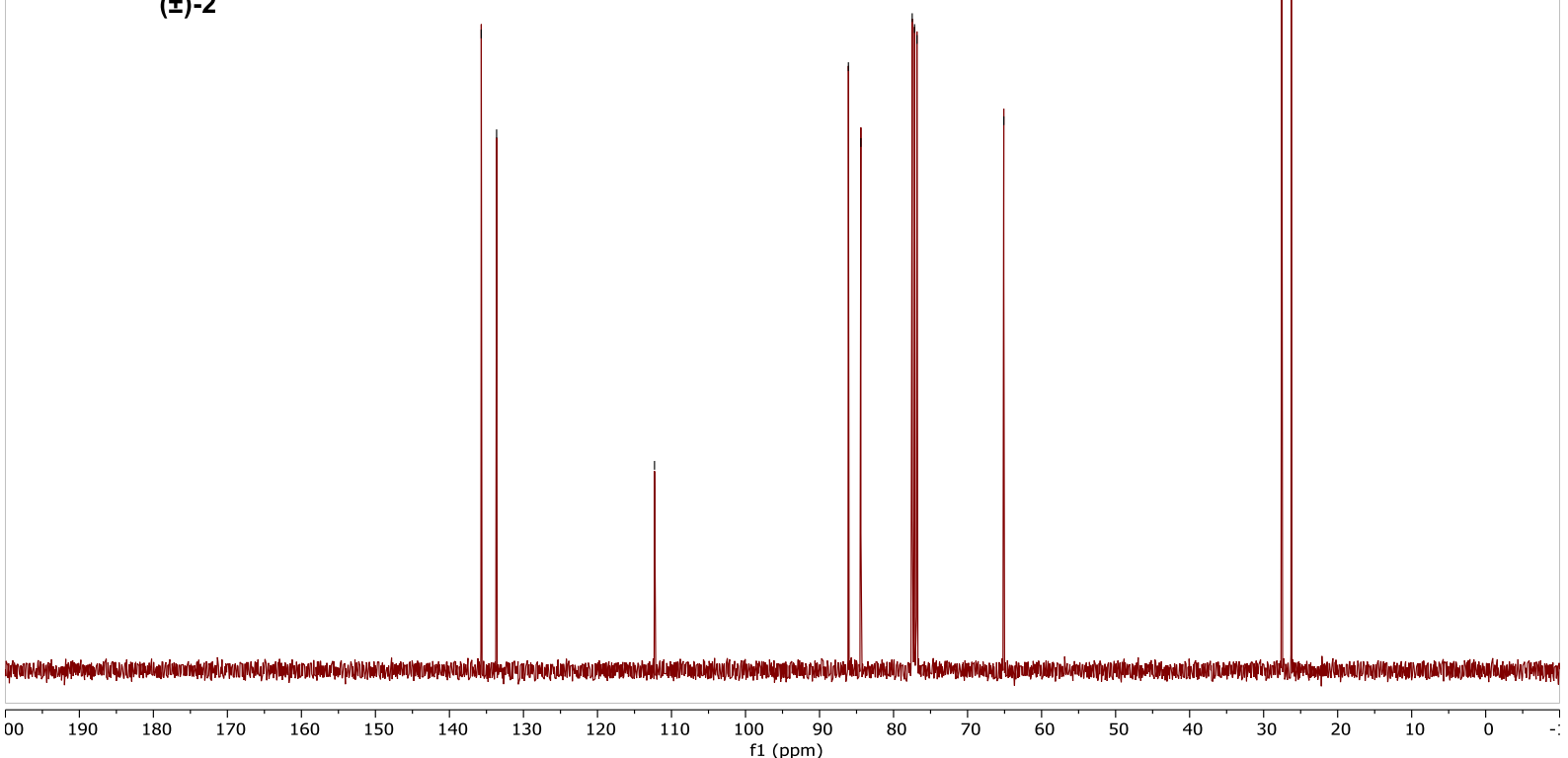

Figure S2: ${ }^{1} \mathrm{H}$ NMR (400 MHz, $\mathrm{CDCl}_{3}$, top) and ${ }^{13} \mathrm{C} \mathrm{NMR}\left(100 \mathrm{MHz}, \mathrm{CDCl}_{3}\right.$, bottom) of compound ( \pm )-2. 


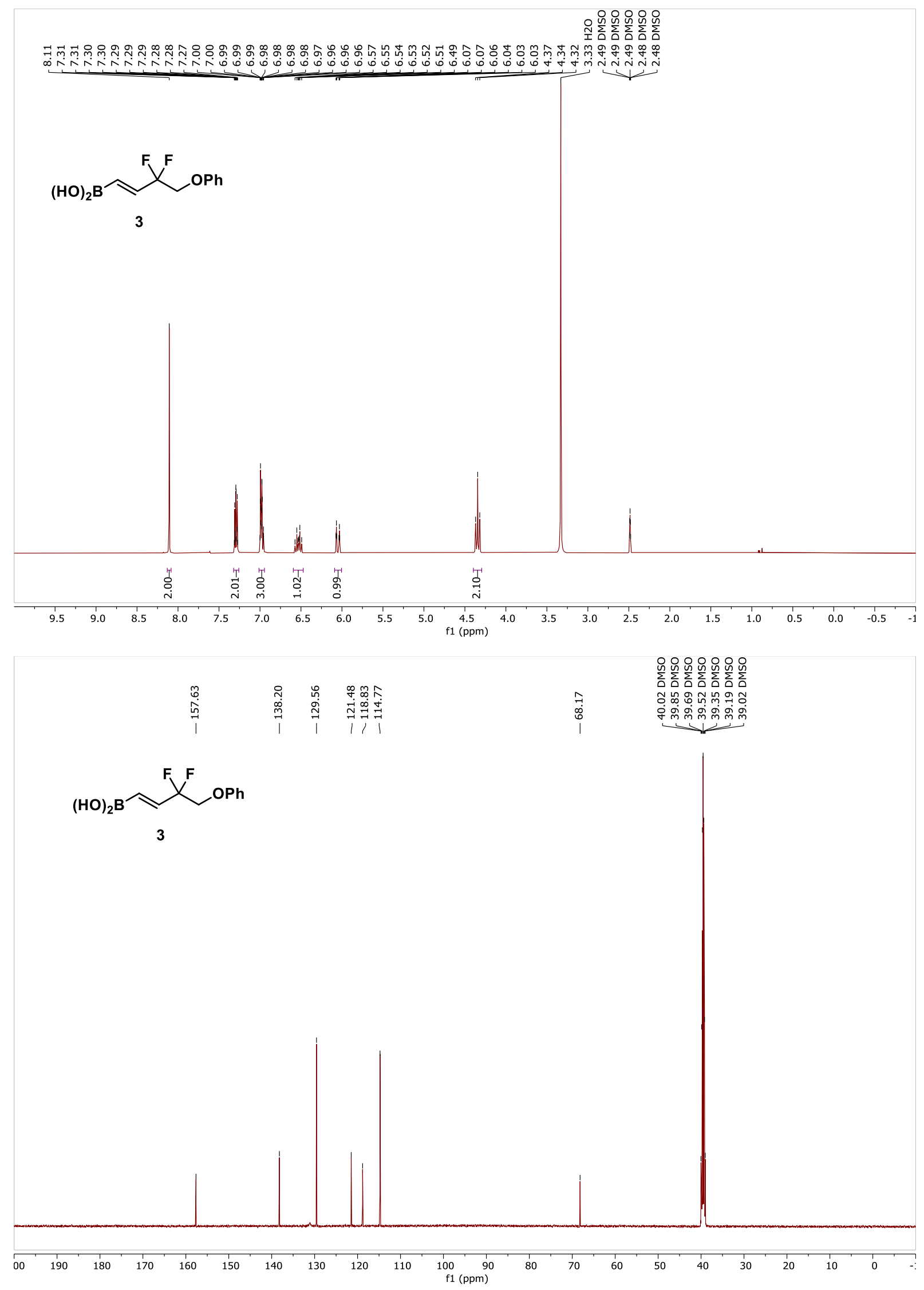

Figure S3: ${ }^{1} \mathrm{H}$ NMR $\left(500 \mathrm{MHz},\left(\mathrm{CD}_{3}\right)_{2} \mathrm{SO}\right.$, top) and ${ }^{13} \mathrm{C} \mathrm{NMR}\left(126 \mathrm{MHz},\left(\mathrm{CD}_{3}\right)_{2} \mathrm{SO}\right.$, bottom) of compound 3 . 


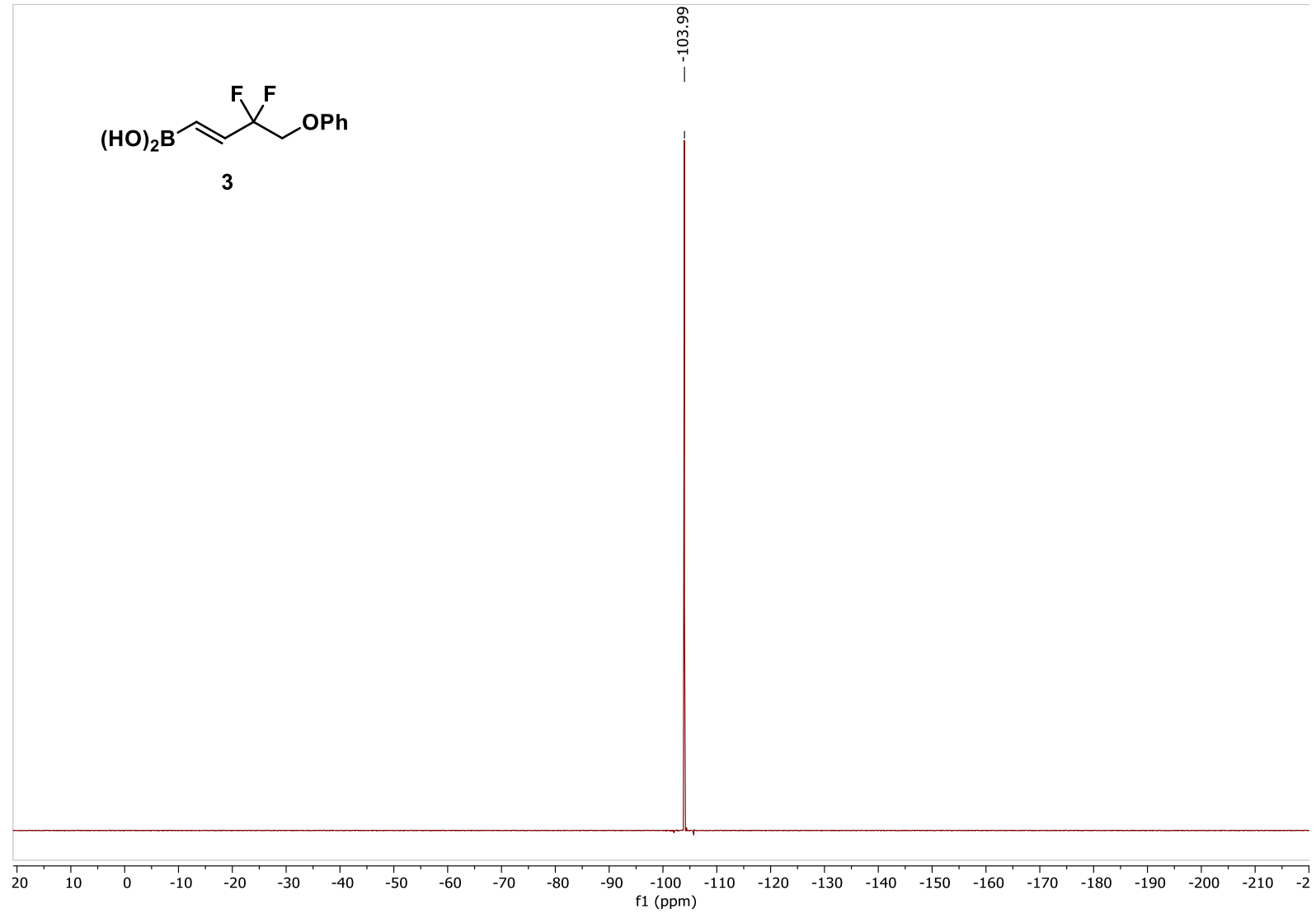

Figure S4: ${ }^{19} \mathrm{~F}$ NMR $\left(471 \mathrm{MHz},\left(\mathrm{CD}_{3}\right)_{2} \mathrm{SO},{ }^{1} \mathrm{H}\right.$ decoupled $)$ of compound 3 . 

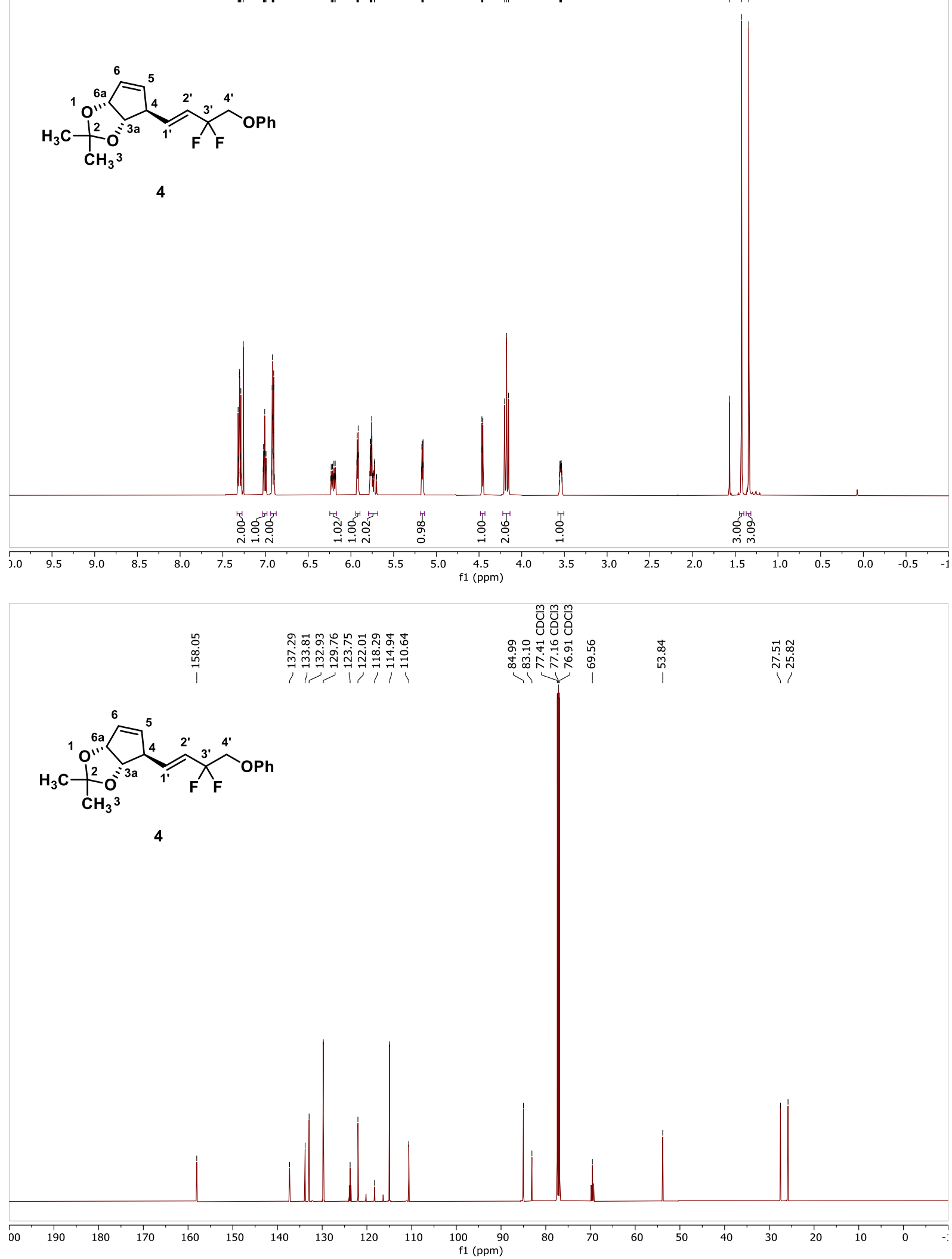

Figure S5: ${ }^{1} \mathrm{H}$ NMR $\left(500 \mathrm{MHz}, \mathrm{CDCl}_{3}\right.$, top) and ${ }^{13} \mathrm{C} \mathrm{NMR} \mathrm{(126} \mathrm{MHz,} \mathrm{CDCl}_{3}$, bottom) of compound 4. 


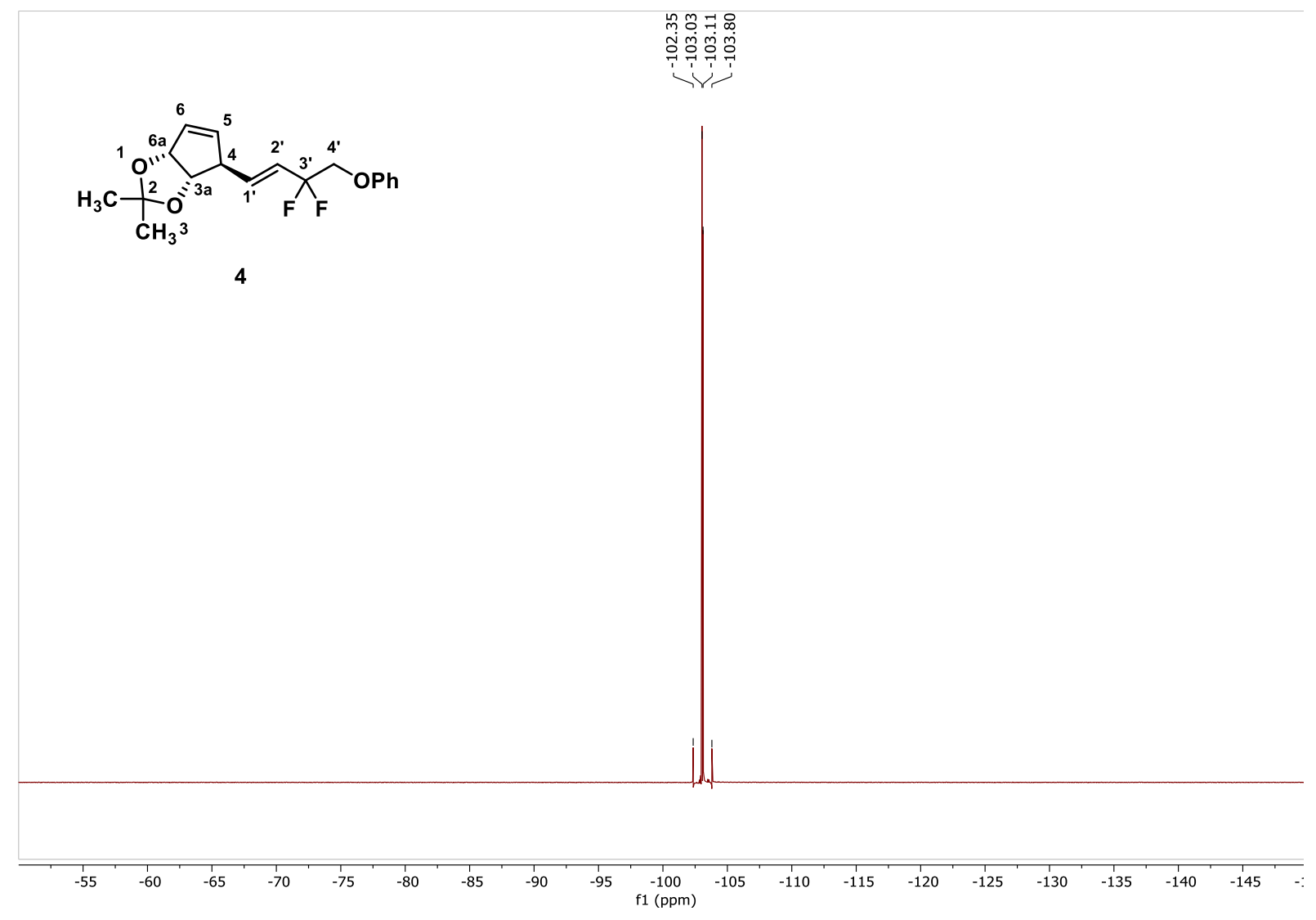

Figure S6: ${ }^{19} \mathrm{~F}$ NMR (471 MHz, $\mathrm{CDCl}_{3},{ }^{1} \mathrm{H}$ decoupled) of compound 4. 

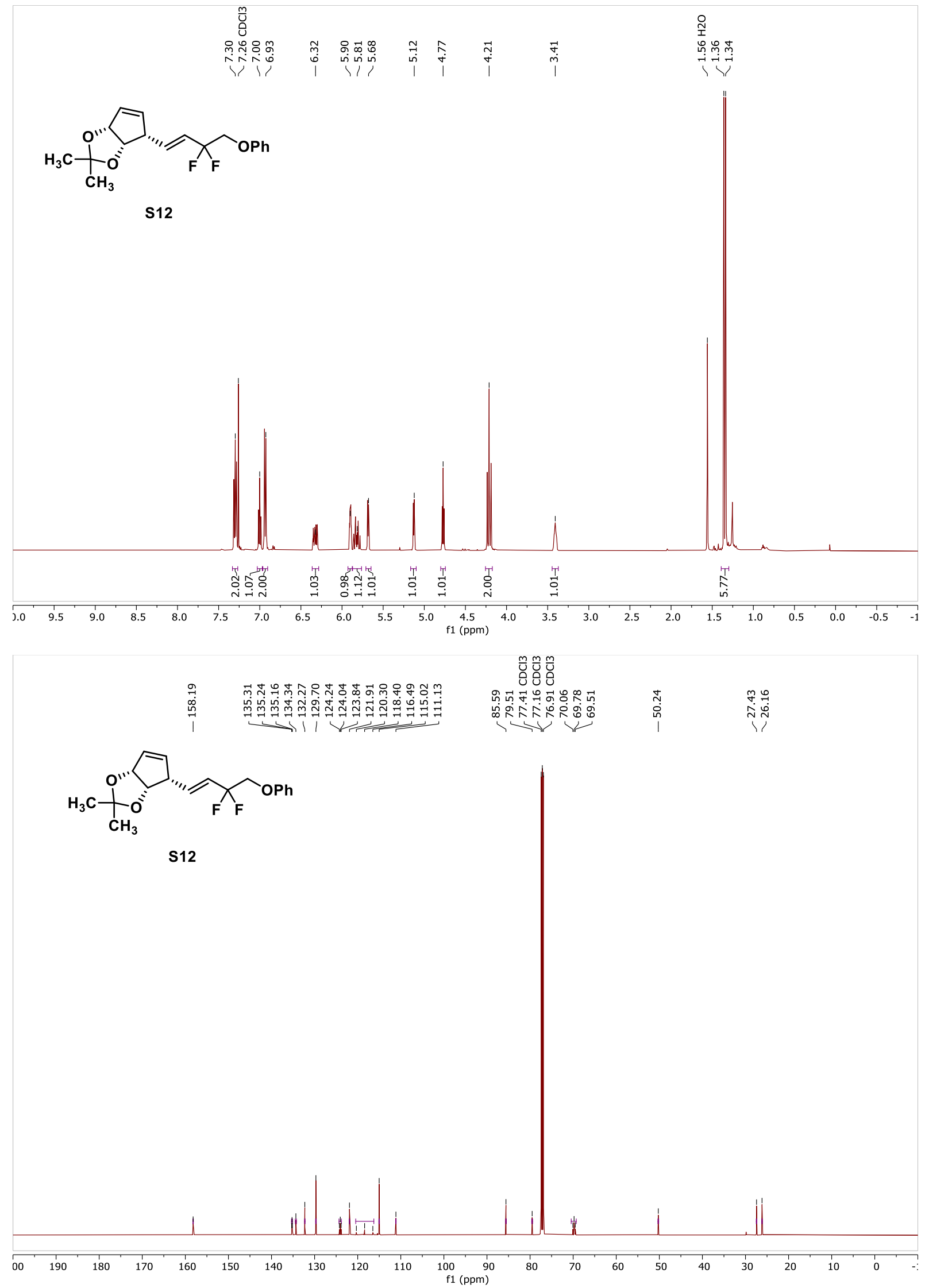

Figure S7: ${ }^{1} \mathrm{H}$ NMR (500 MHz, $\mathrm{CDCl}_{3}$, top) and ${ }^{13} \mathrm{C} \mathrm{NMR}\left(126 \mathrm{MHz}, \mathrm{CDCl}_{3}\right.$, bottom) of compound S12. 


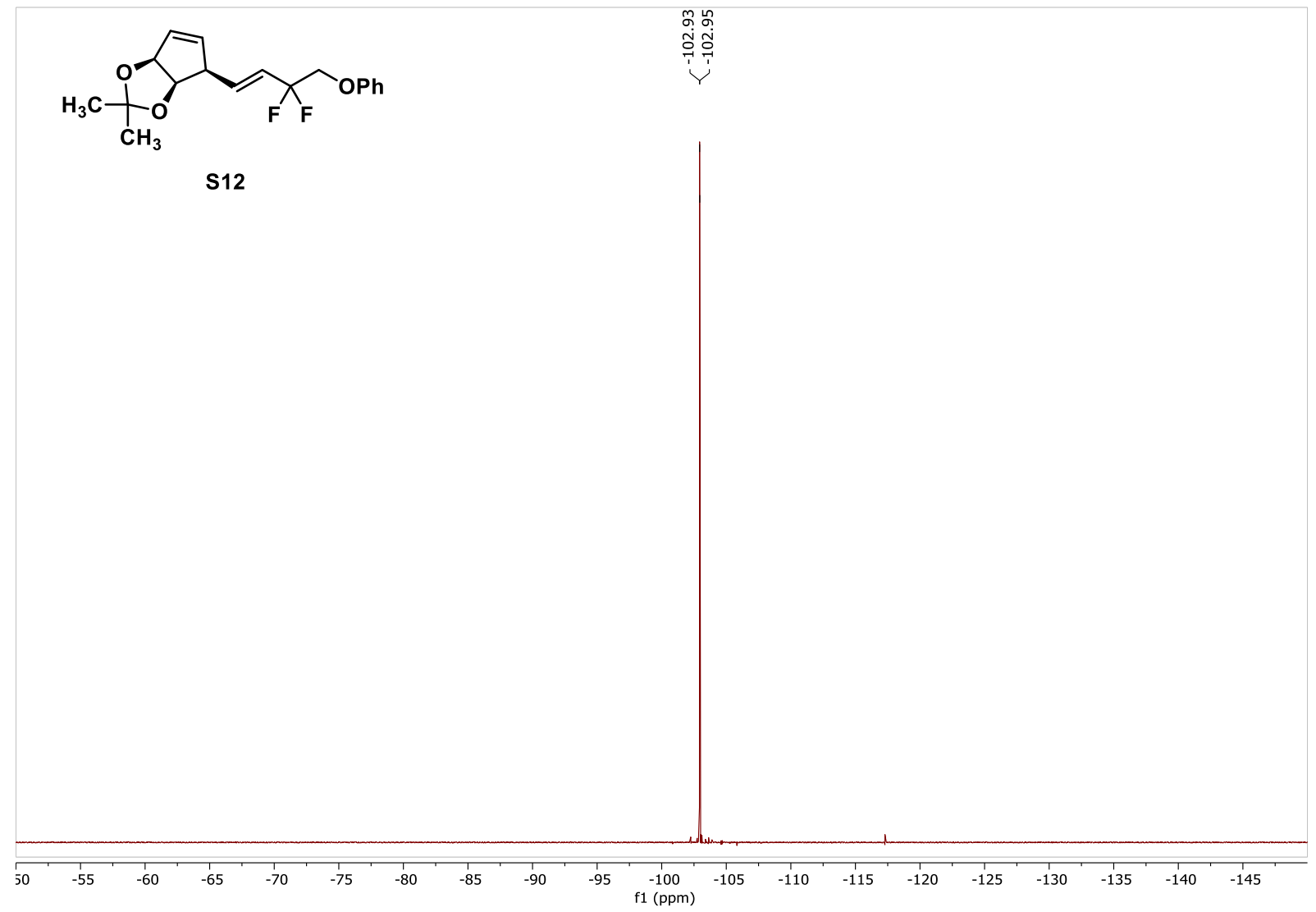

Figure S8: ${ }^{19} \mathrm{~F}$ NMR $\left(377 \mathrm{MHz}, \mathrm{CDCl}_{3},{ }^{1} \mathrm{H}\right.$ decoupled) of compound $\mathbf{S 1 2}$. 

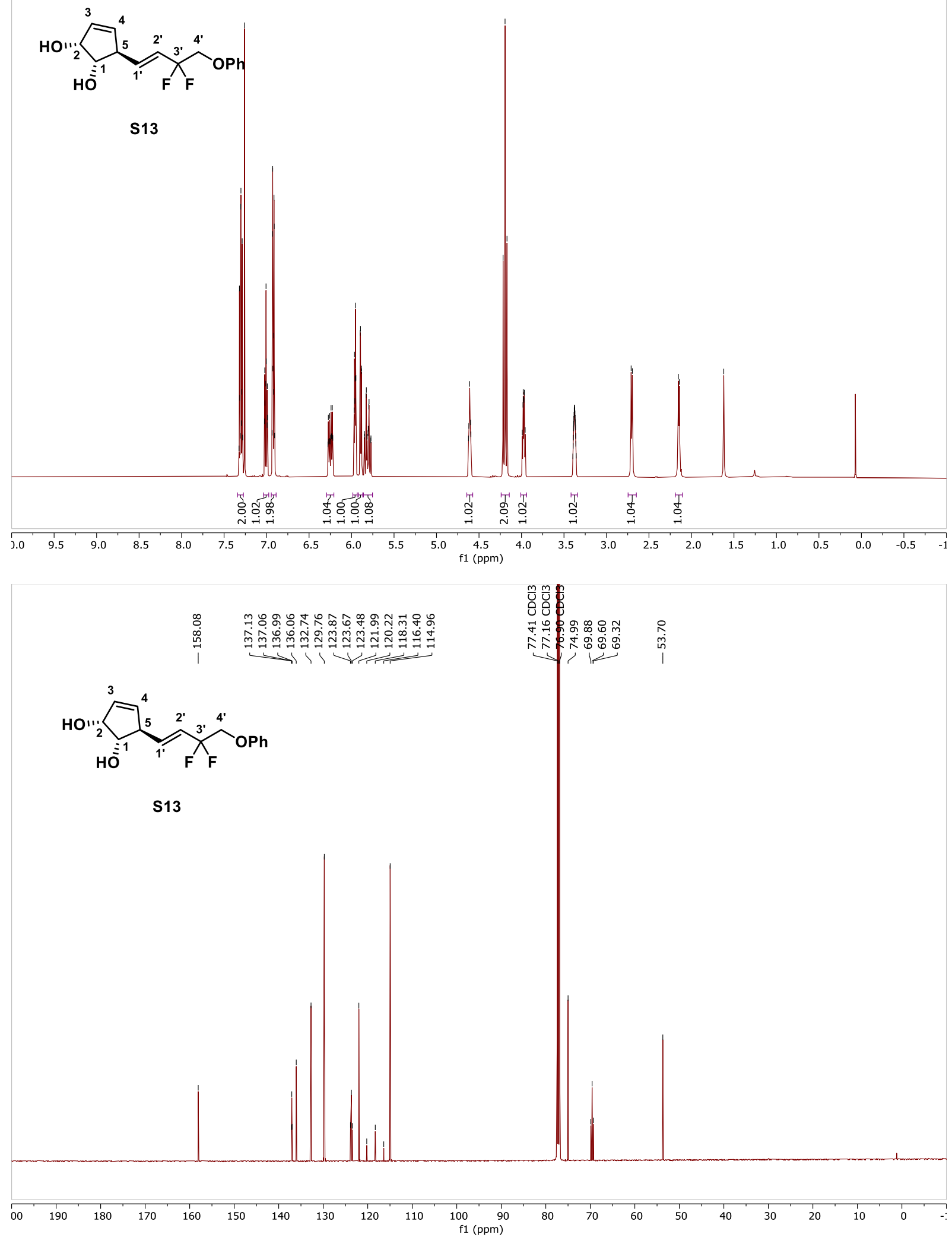

Figure S9: ${ }^{1} \mathrm{H}$ NMR (500 MHz, $\mathrm{CDCl}_{3}$, top) and ${ }^{13} \mathrm{C} \mathrm{NMR}\left(126 \mathrm{MHz}, \mathrm{CDCl}_{3}\right.$, bottom) of compound S13. 


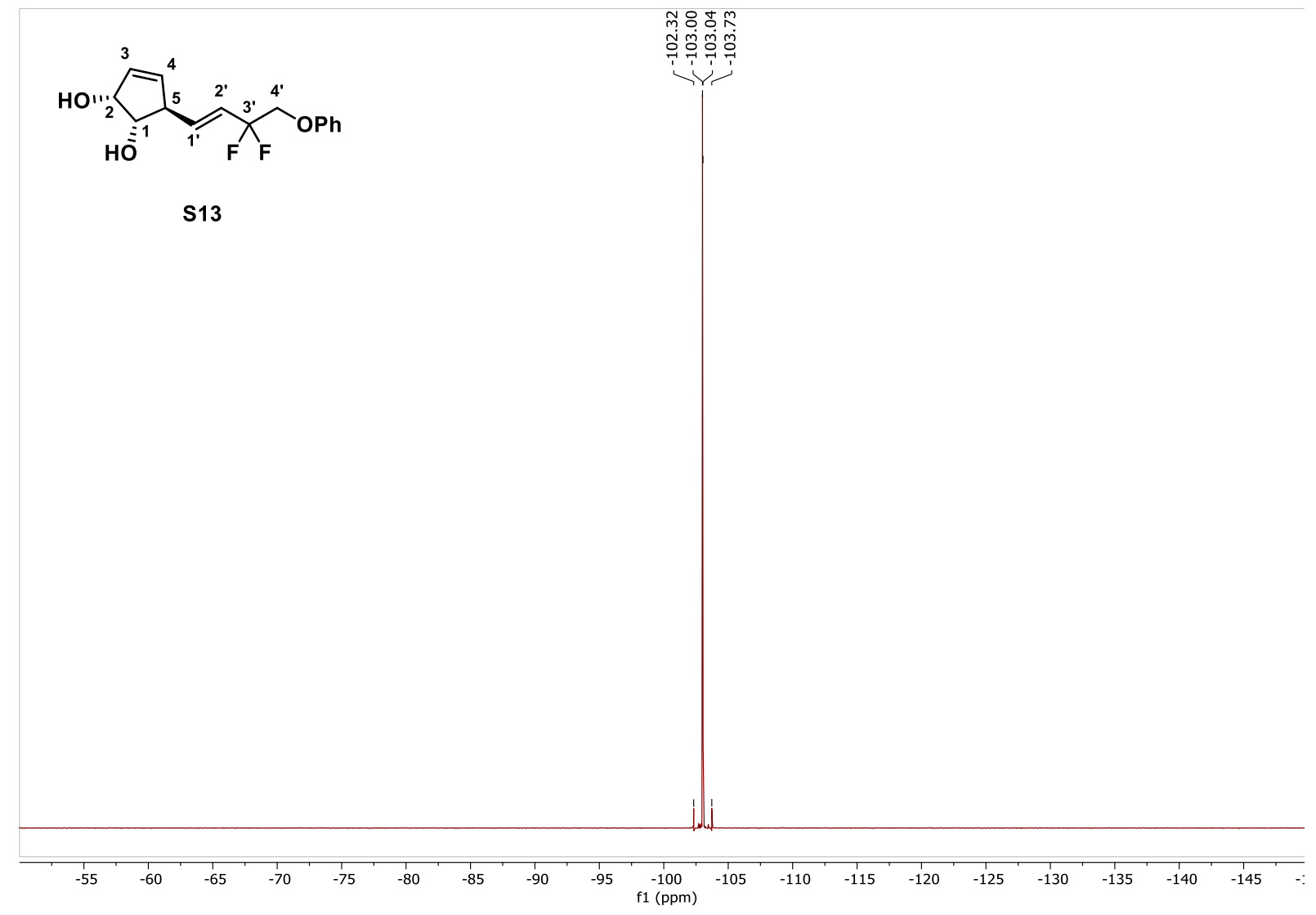

Figure S10: ${ }^{19} \mathrm{~F}$ NMR $\left(377 \mathrm{MHz}, \mathrm{CDCl}_{3},{ }^{1} \mathrm{H}\right.$ decoupled) of compound $\mathbf{S 1 3}$. 
<smiles>O=C1O[C@H]2C=CC(/C=C/C(F)(F)COc3ccccc3)[C@H]2O1</smiles>

5

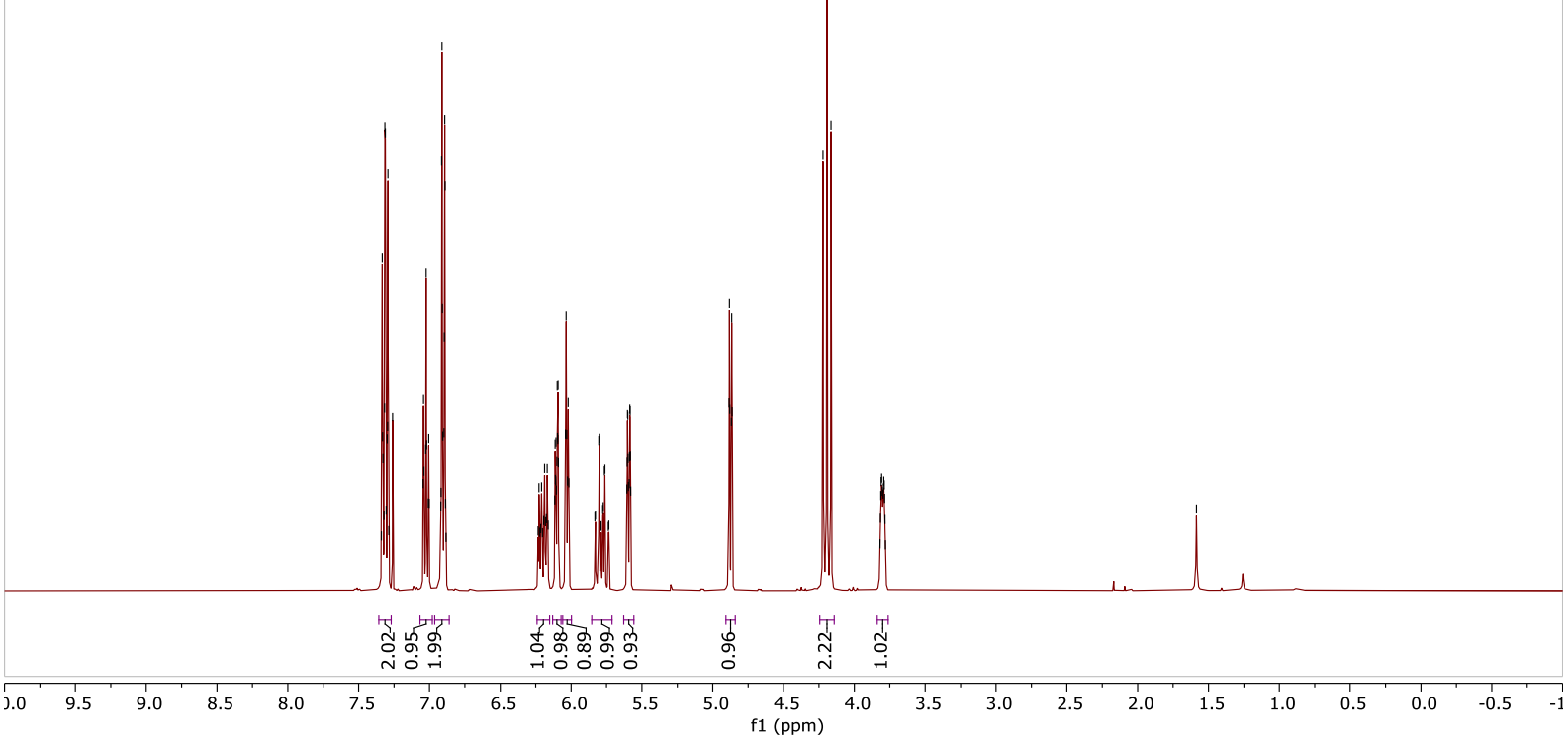

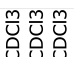

क.

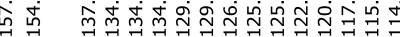

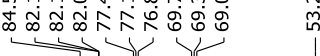
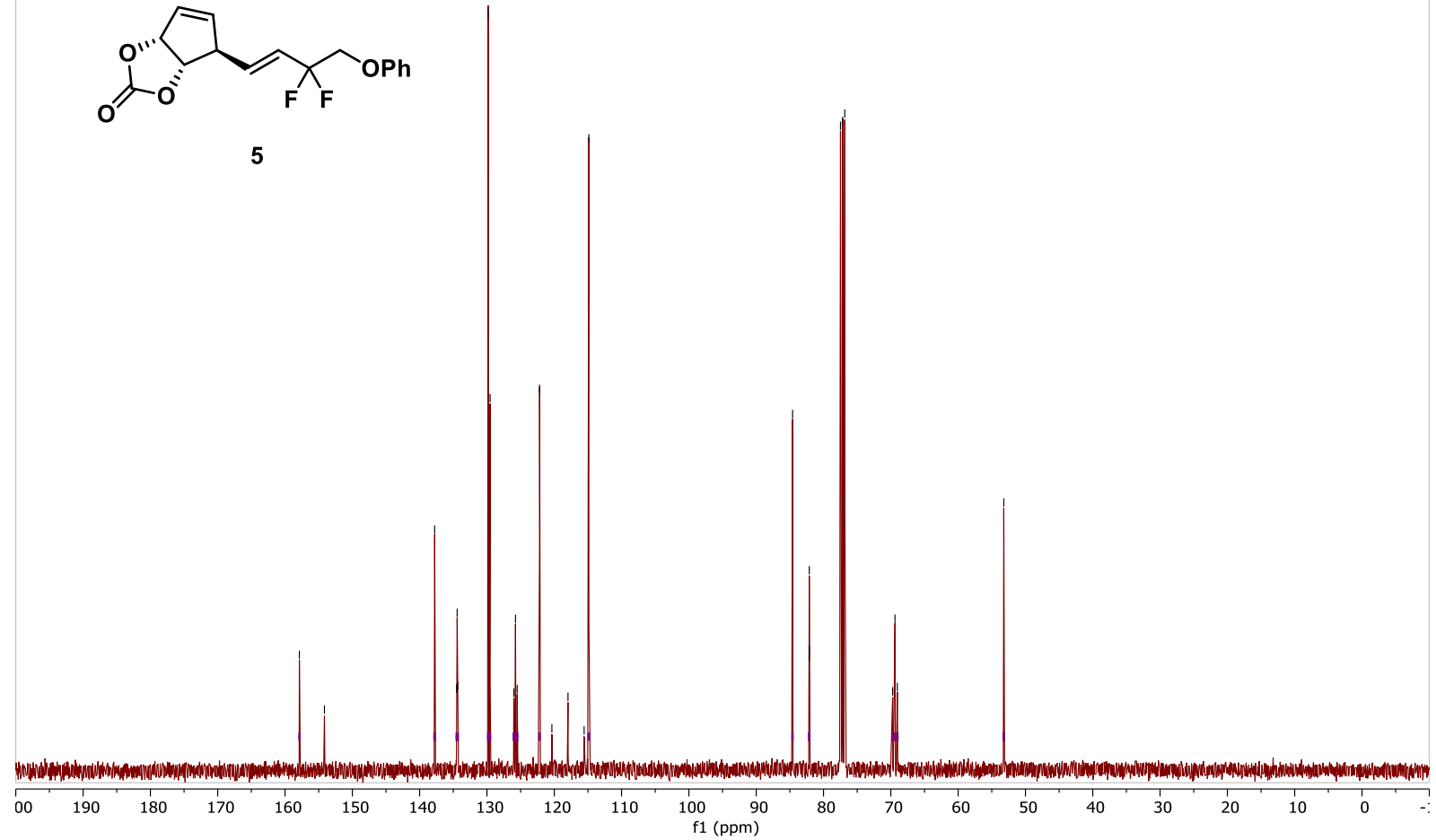

Figure S11: ${ }^{1} \mathrm{H}$ NMR (400 MHz, $\mathrm{CDCl}_{3}$, top) and ${ }^{13} \mathrm{C} \mathrm{NMR} \mathrm{(101} \mathrm{MHz}, \mathrm{CDCl}_{3}$, bottom) of compound 5. 


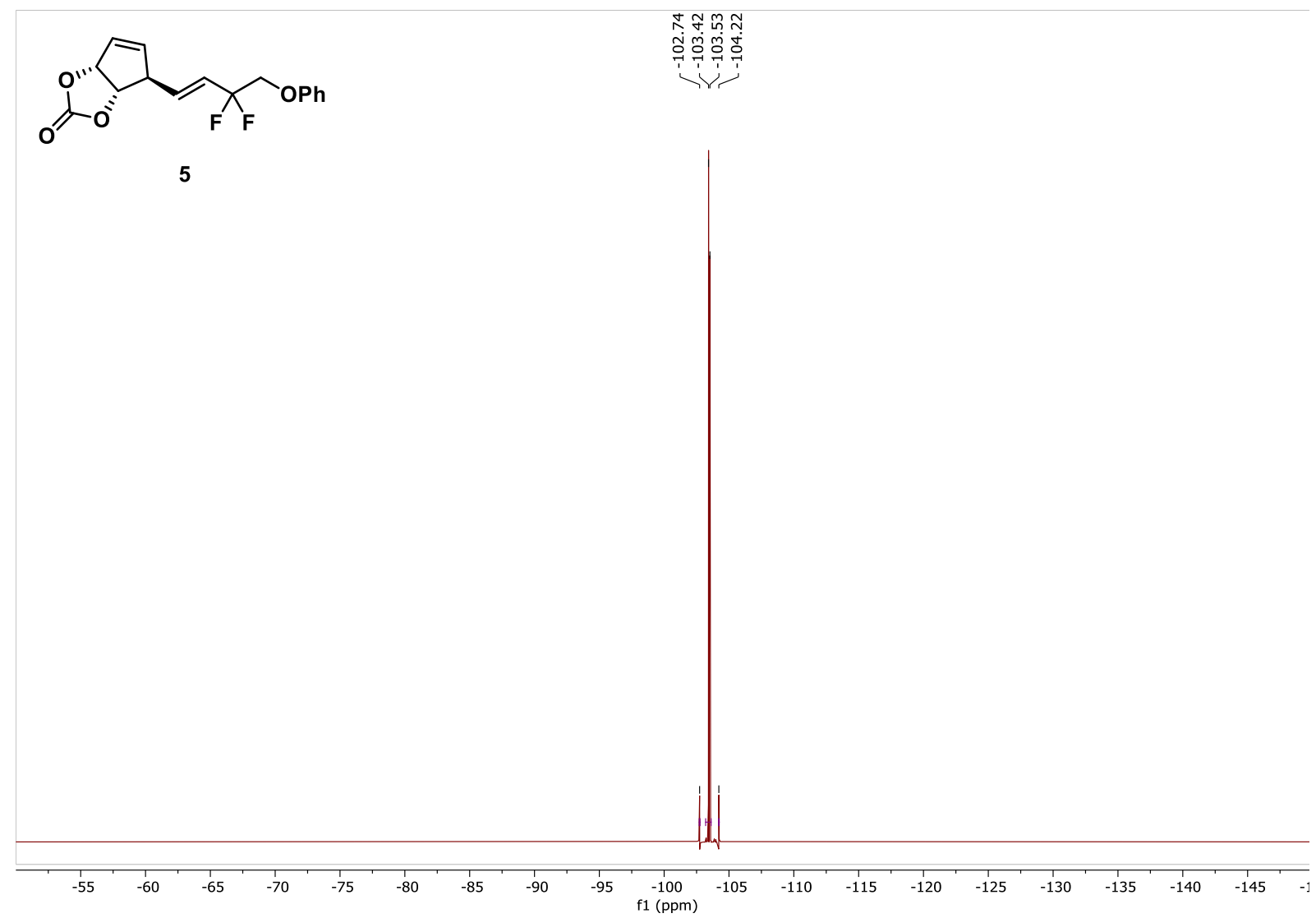

Figure S12: ${ }^{17} \mathrm{~F}$ NMR $\left(377 \mathrm{MHz}, \mathrm{CDCl}_{3},{ }^{1} \mathrm{H}\right.$ decoupled) of compound 5. 

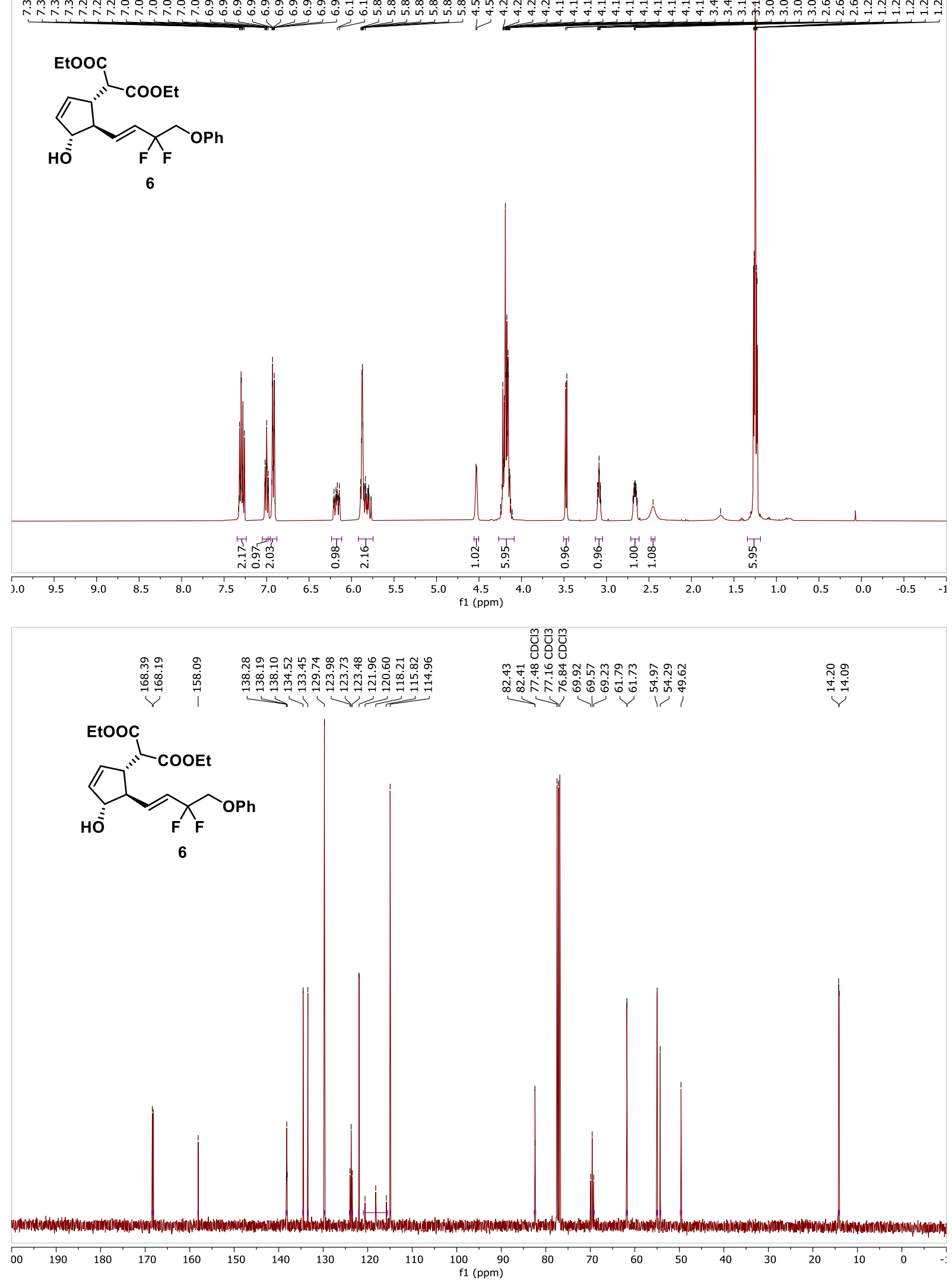

Figure S13: ${ }^{1} \mathrm{H}$ NMR (400 MHz, $\mathrm{CDCl}_{3}$, top) and ${ }^{13} \mathrm{C} \mathrm{NMR} \mathrm{(101} \mathrm{MHz}, \mathrm{CDCl}_{3}$, bottom) of compound 6. 


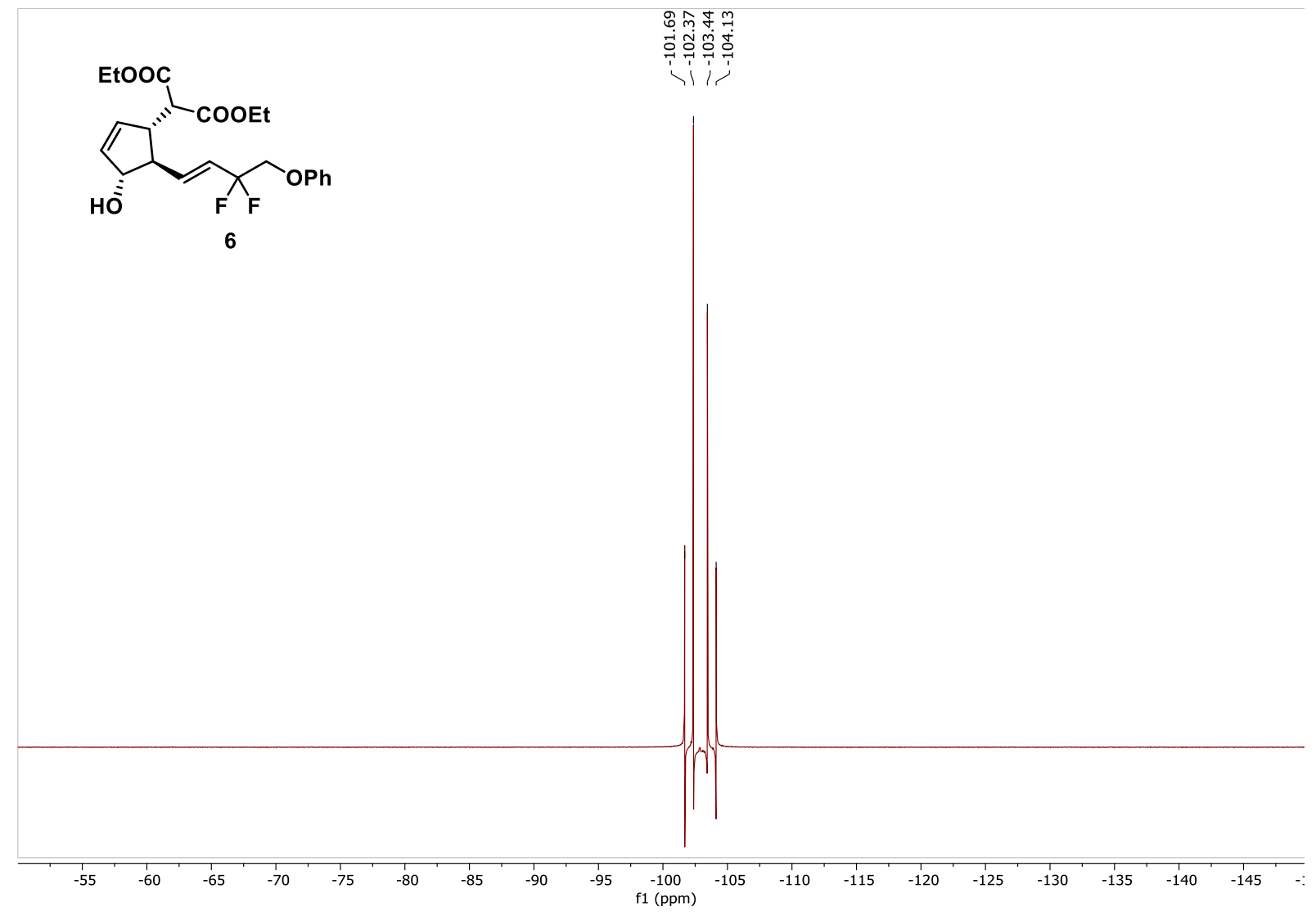

Figure S14: ${ }^{19} \mathrm{~F}$ NMR ( $377 \mathrm{MHz}, \mathrm{CDCl}_{3},{ }^{1} \mathrm{H}$ decoupled) of compound 6. 


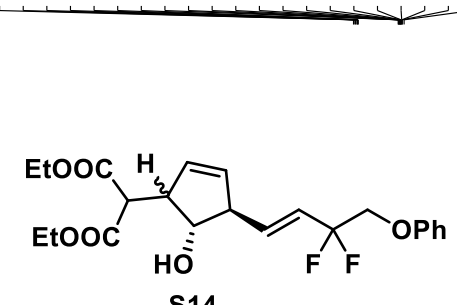

S14
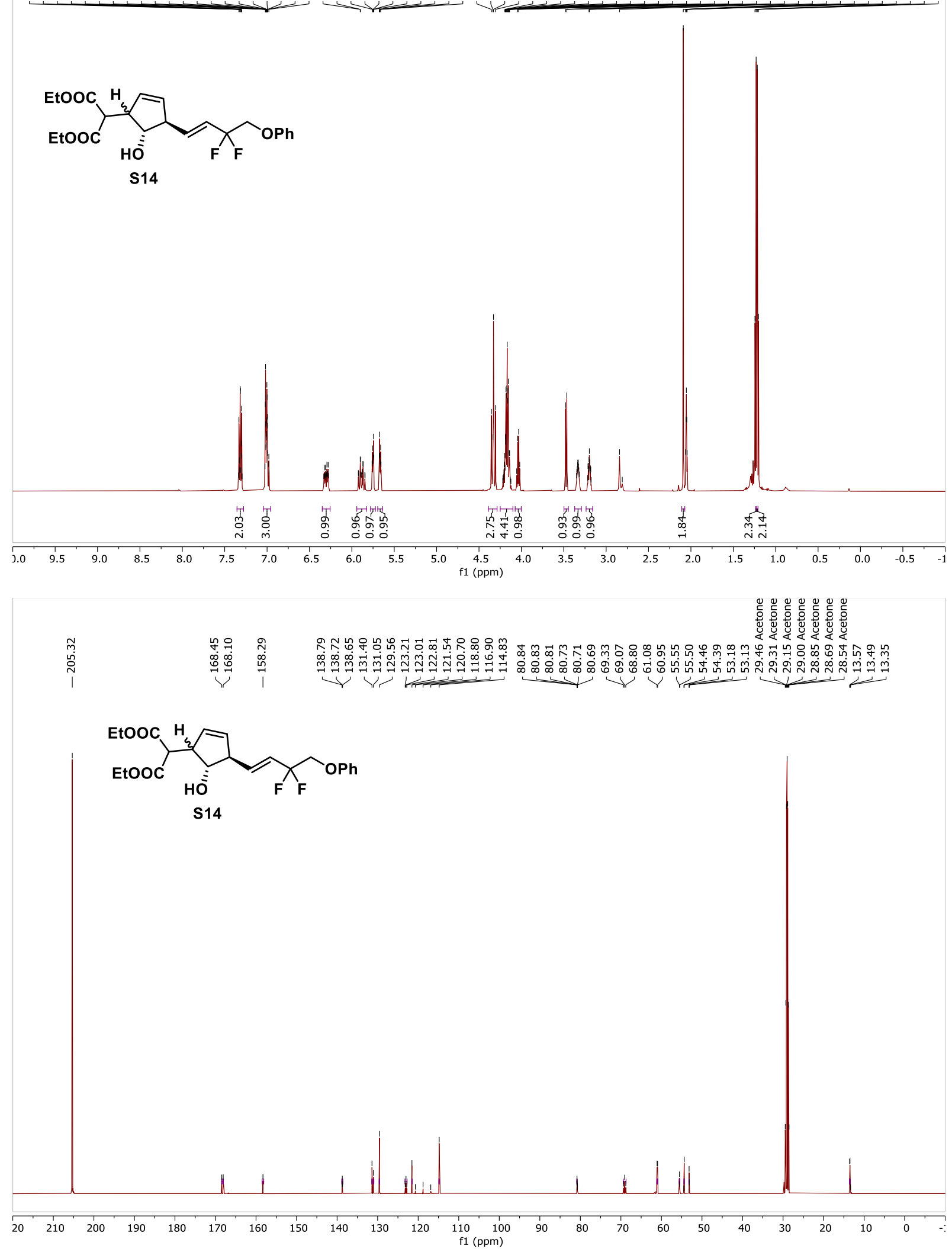

Figure S15: ${ }^{1} \mathrm{H}$ NMR $\left(500 \mathrm{MHz},\left(\mathrm{CD}_{3}\right)_{2} \mathrm{CO}\right.$, top) and ${ }^{13} \mathrm{C}$ NMR $\left(126 \mathrm{MHz},\left(\mathrm{CD}_{3}\right)_{2} \mathrm{CO}\right.$, bottom) of compound S14. 


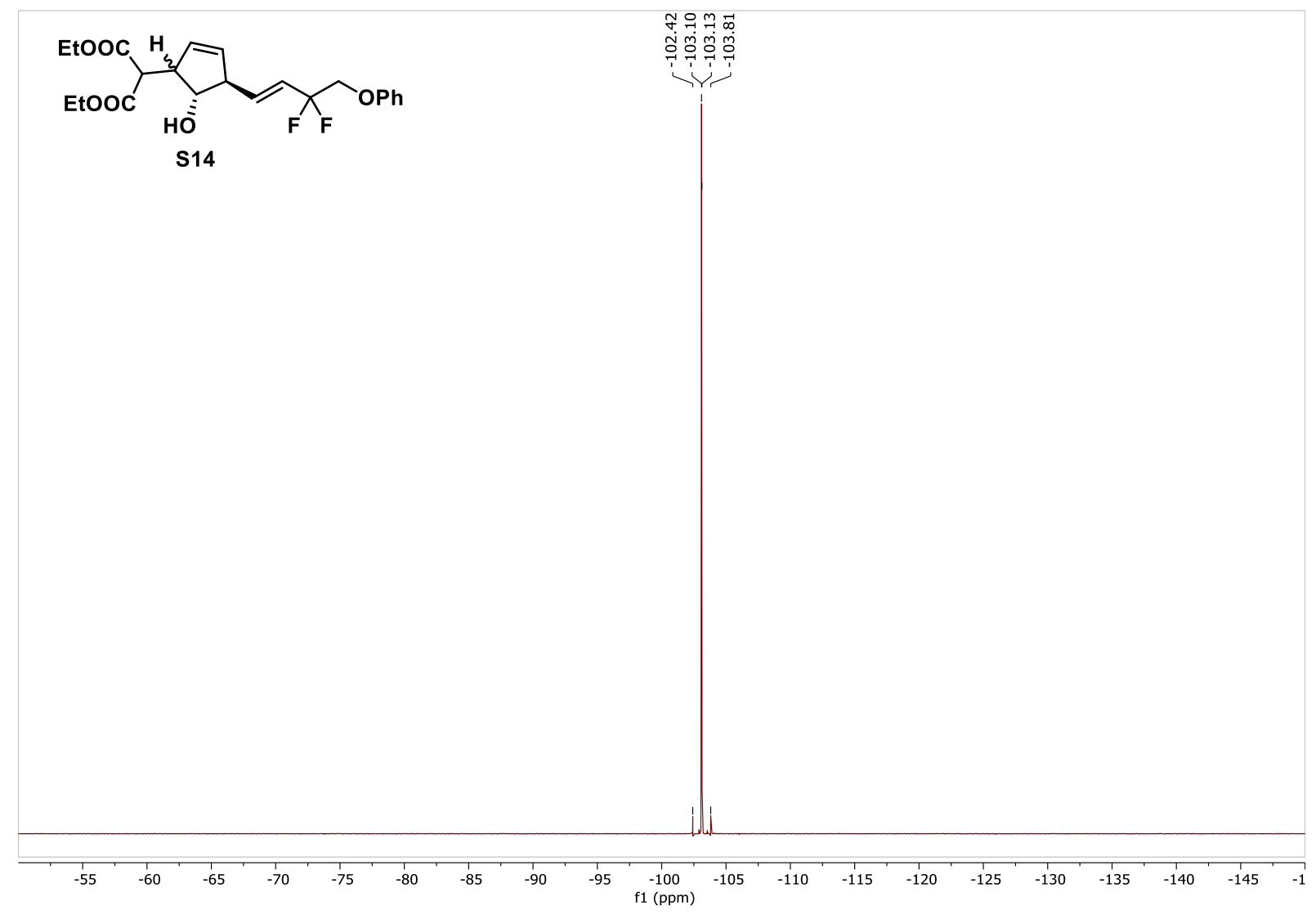

Figure S16: ${ }^{1} \mathrm{H}$ NMR $\left(377 \mathrm{MHz},\left(\mathrm{CD}_{3}\right)_{2} \mathrm{CO},{ }^{1} \mathrm{H}\right.$ decoupled $)$ of compound $\mathbf{S 1 4}$. 

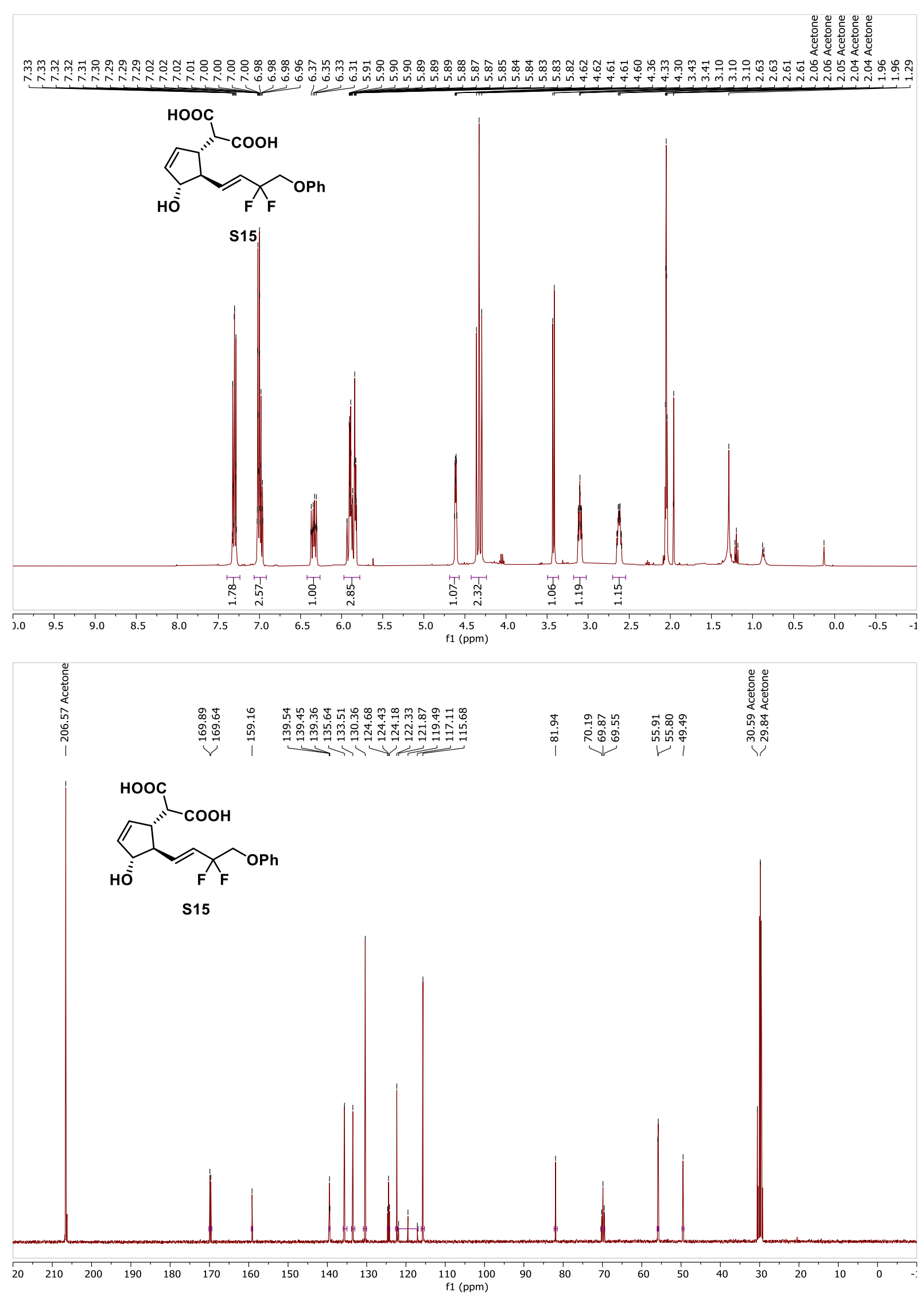

Figure S17: ${ }^{1} \mathrm{H}$ NMR (400 MHz, $\left(\mathrm{CD}_{3}\right)_{2} \mathrm{CO}$, top) and ${ }^{13} \mathrm{C} \mathrm{NMR}\left(101 \mathrm{MHz},\left(\mathrm{CD}_{3}\right)_{2} \mathrm{CO}\right.$, bottom) of compound S15. 


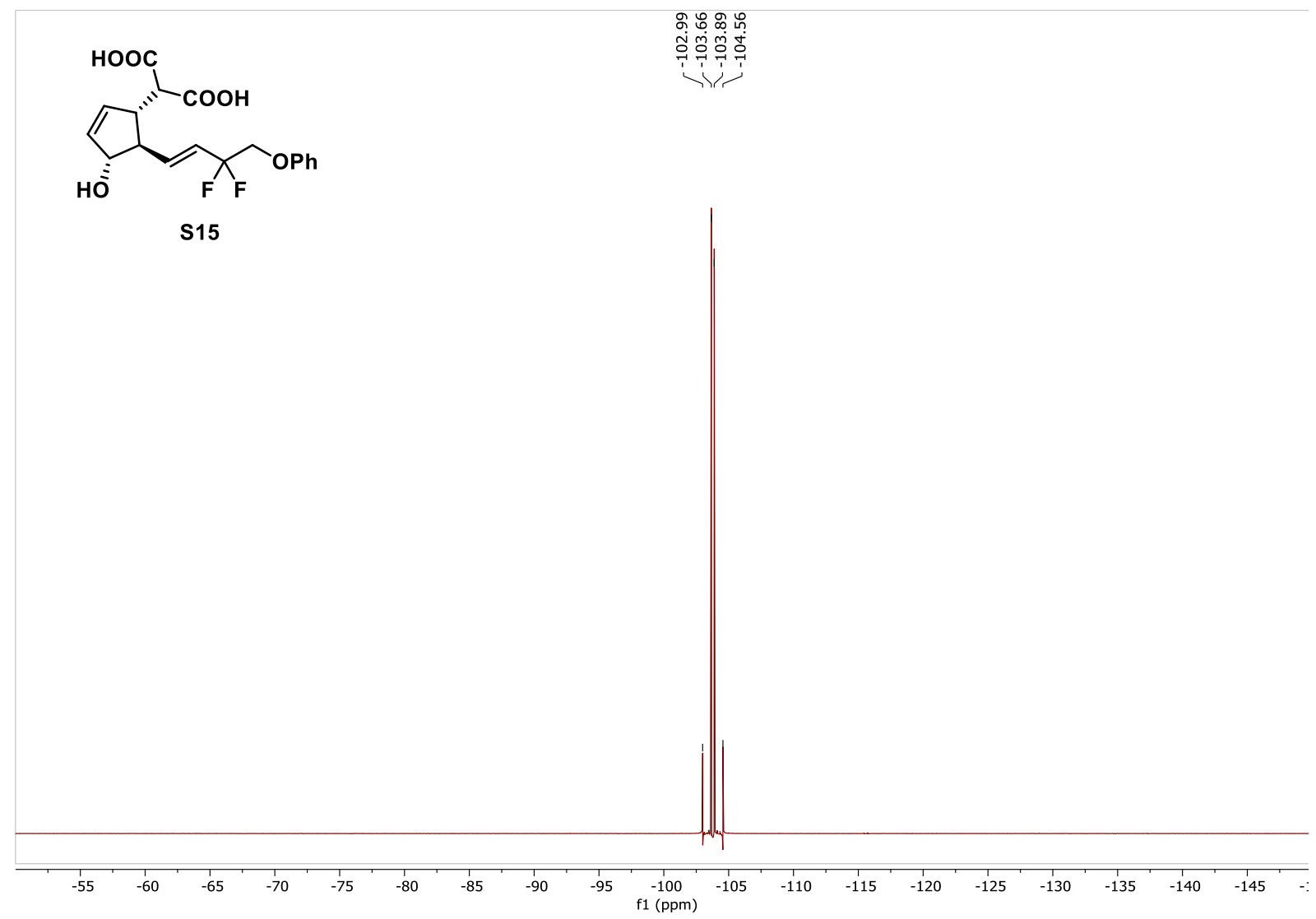

Figure S18: ${ }^{19} \mathrm{~F}$ NMR $\left(377 \mathrm{MHz},\left(\mathrm{CD}_{3}\right)_{2} \mathrm{CO},{ }^{1} \mathrm{H}\right.$ decoupled $)$ of compound $\mathbf{S 1 5}$. 

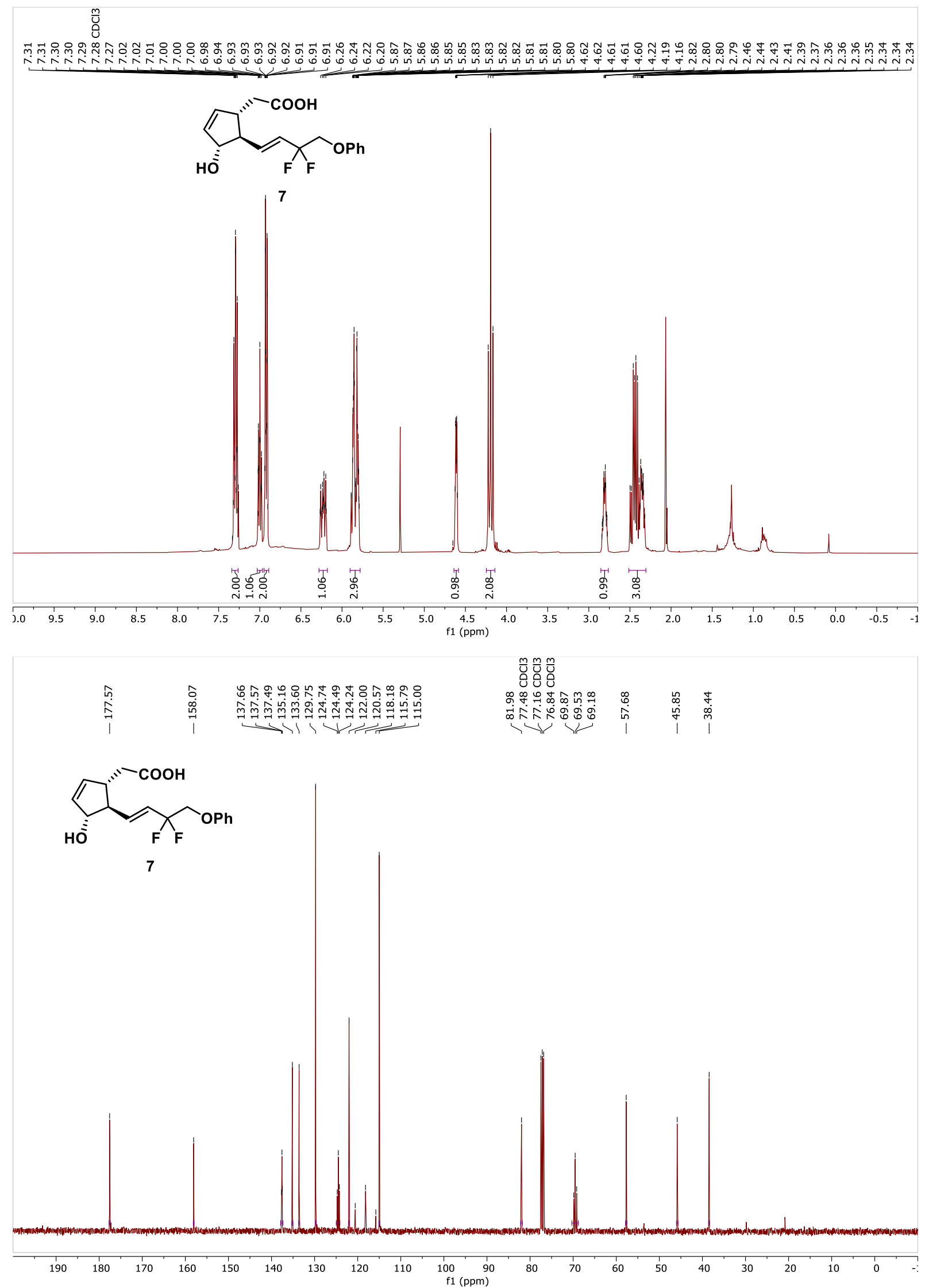

Figure S19: ${ }^{1} \mathrm{H}$ NMR (400 MHz, $\mathrm{CDCl}_{3}$, top) and ${ }^{13} \mathrm{C} \mathrm{NMR}\left(101 \mathrm{MHz}, \mathrm{CDCl}_{3}\right.$, bottom) of compound 7. 


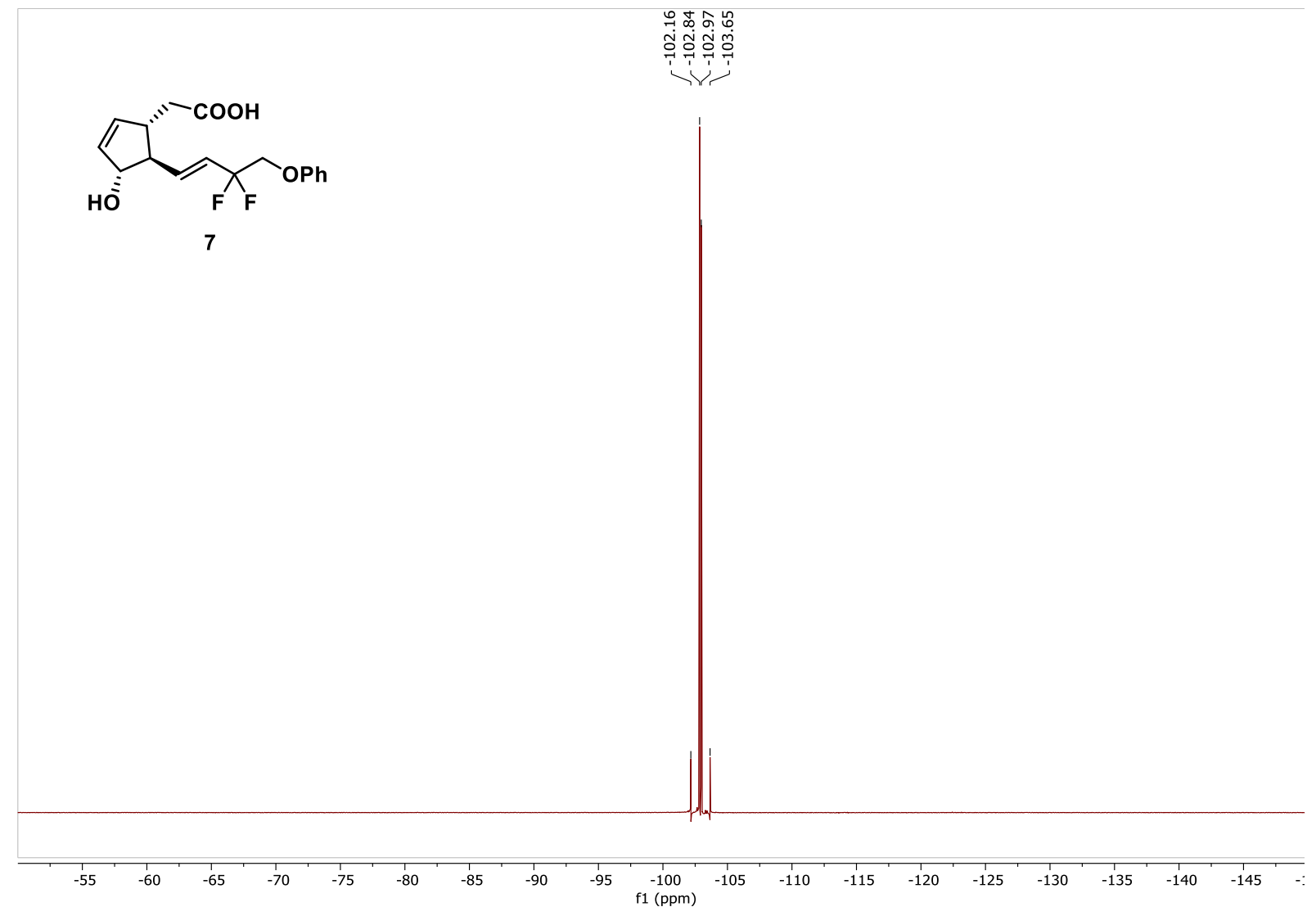

Figure S20: ${ }^{19} \mathrm{~F}$ NMR $\left(377 \mathrm{MHz}, \mathrm{CDCl}_{3},{ }^{1} \mathrm{H}\right.$ decoupled) of compound 7. 
<smiles>O=C1C[C@H]2[C@H](O1)[C@H](O)[C@H](I)[C@H]2/C=C/C(F)(F)COc1ccccc1</smiles>
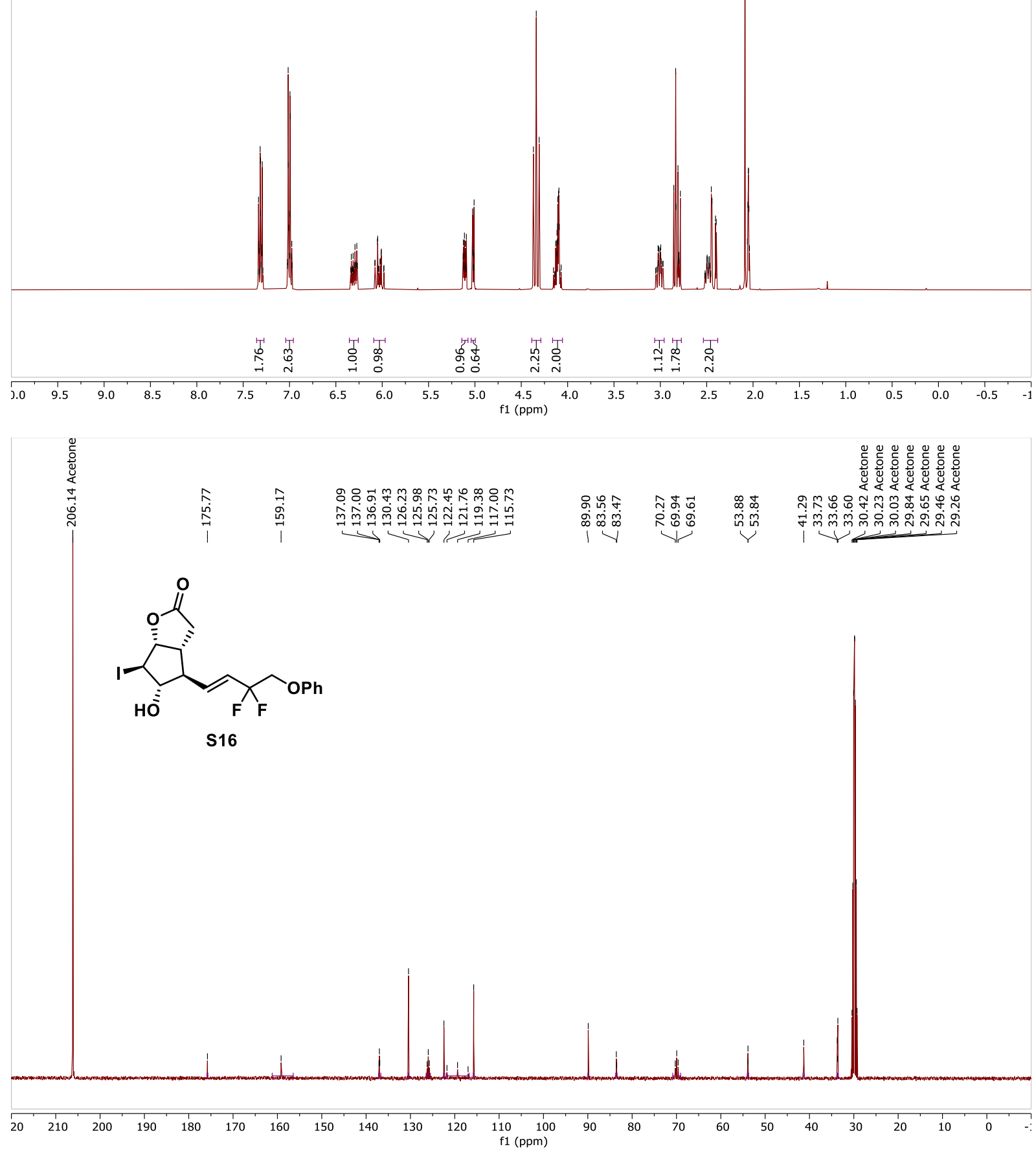

Figure S21: ${ }^{1} \mathrm{H}$ NMR (400 MHz, $\left(\mathrm{CD}_{3}\right)_{2} \mathrm{CO}$, top) and ${ }^{13} \mathrm{C}$ NMR $\left(101 \mathrm{MHz},\left(\mathrm{CD}_{3}\right)_{2} \mathrm{CO}\right.$, bottom) of compound S16. 


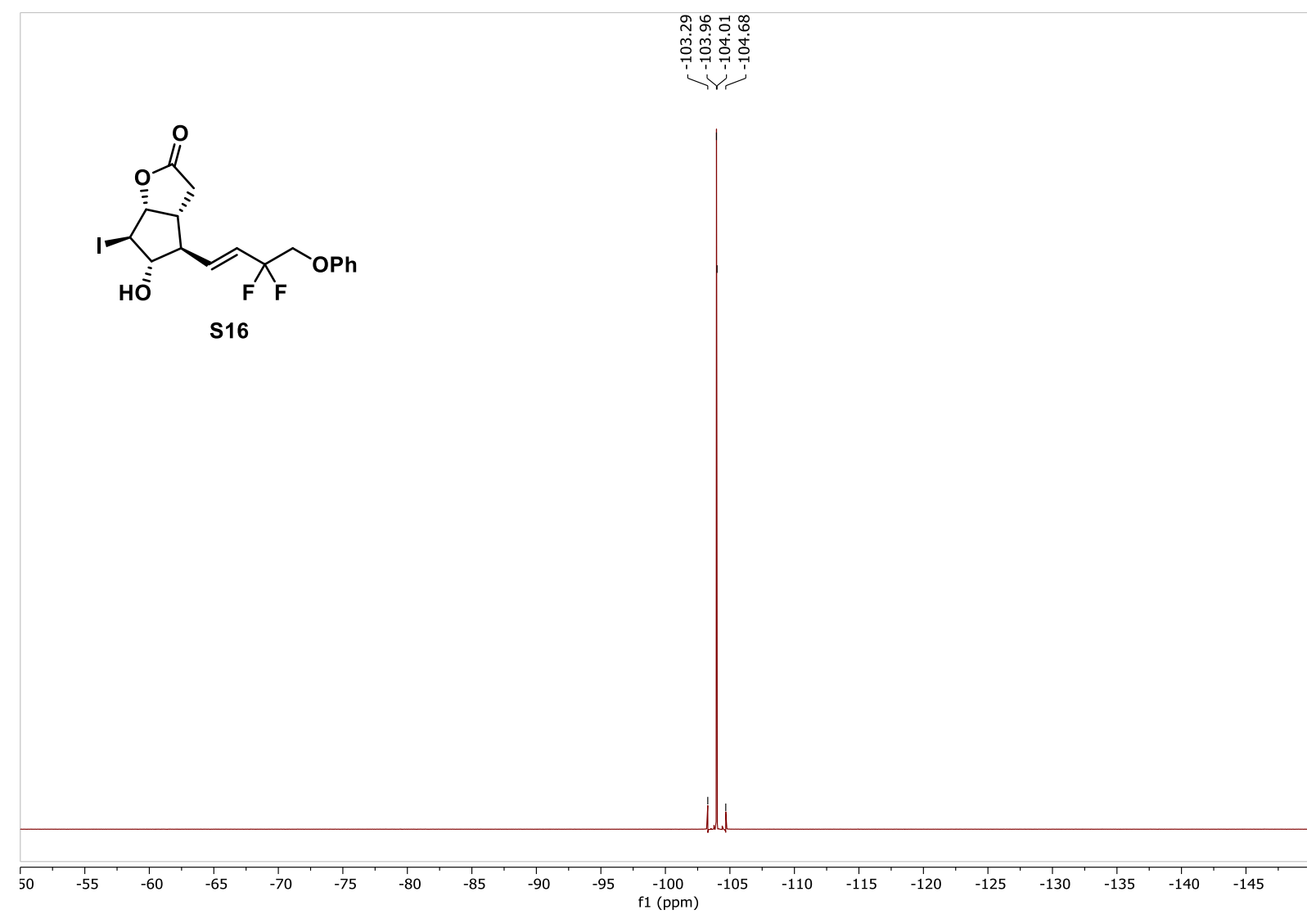

Figure S22: ${ }^{19} \mathrm{~F}$ NMR $\left(377 \mathrm{MHz},\left(\mathrm{CD}_{3}\right)_{2} \mathrm{CO},{ }^{1} \mathrm{H}\right.$ decoupled $)$ of compound $\mathbf{S 1 6}$. 

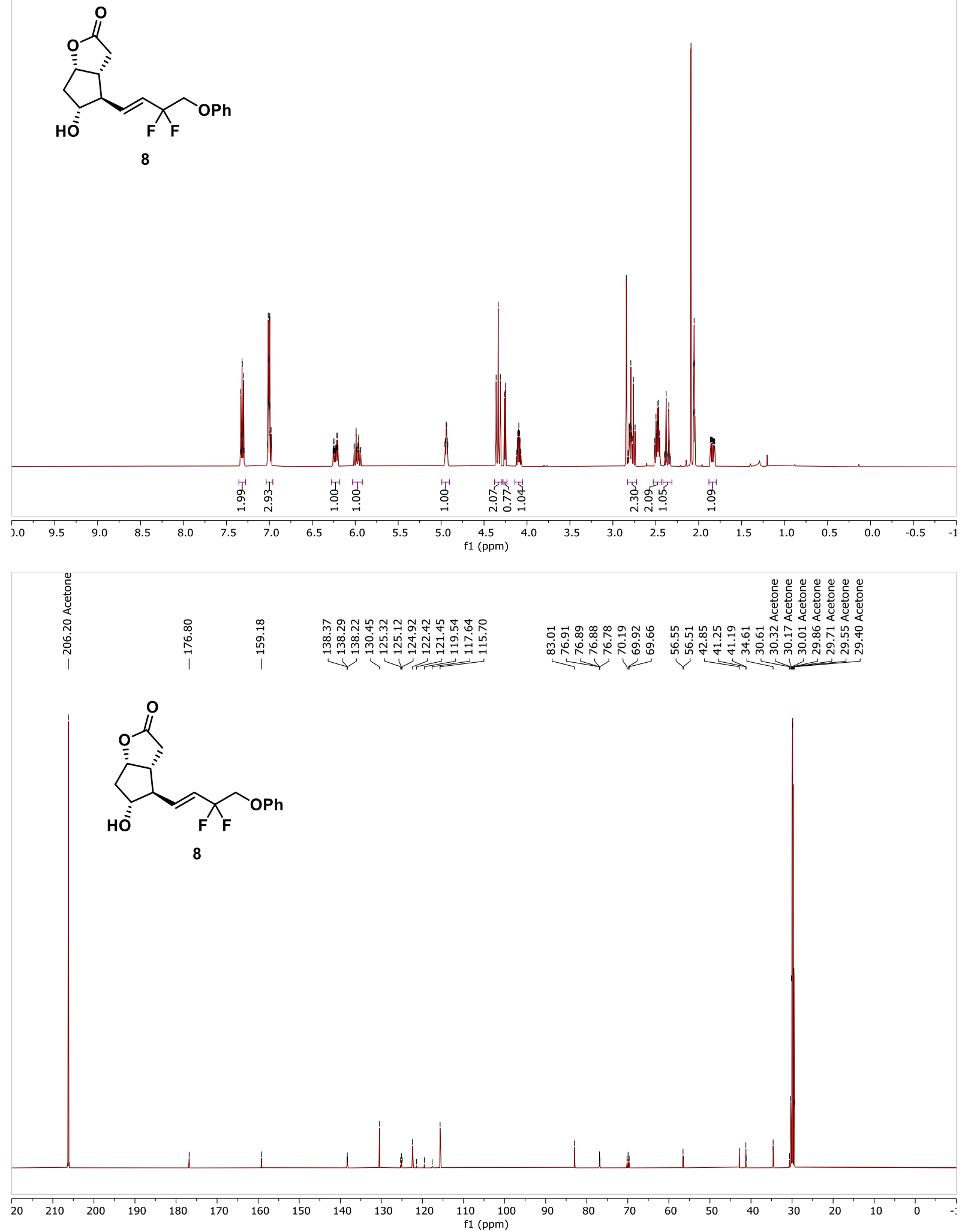

Figure S23: ${ }^{1} \mathrm{H}$ NMR $\left(400 \mathrm{MHz},\left(\mathrm{CD}_{3}\right)_{2} \mathrm{CO}\right.$, top) and ${ }^{13} \mathrm{C}$ NMR $\left(101 \mathrm{MHz},\left(\mathrm{CD}_{3}\right)_{2} \mathrm{CO}\right.$, bottom) of compound 8 . 


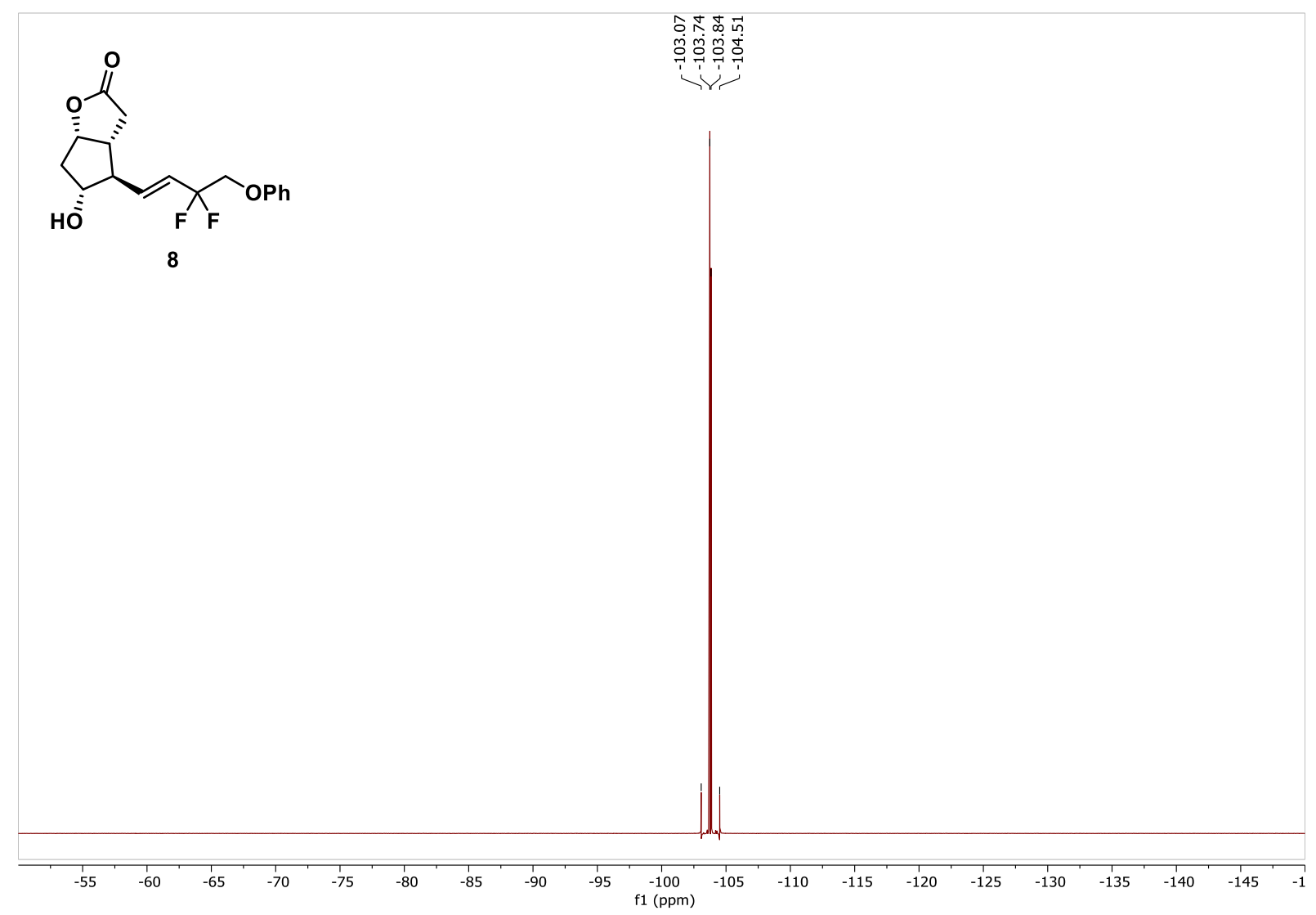

Figure S24: ${ }^{19} \mathrm{~F}$ NMR $\left(377 \mathrm{MHz},\left(\mathrm{CD}_{3}\right)_{2} \mathrm{CO},{ }^{1} \mathrm{H}\right.$ decoupled) of compound 8 . 

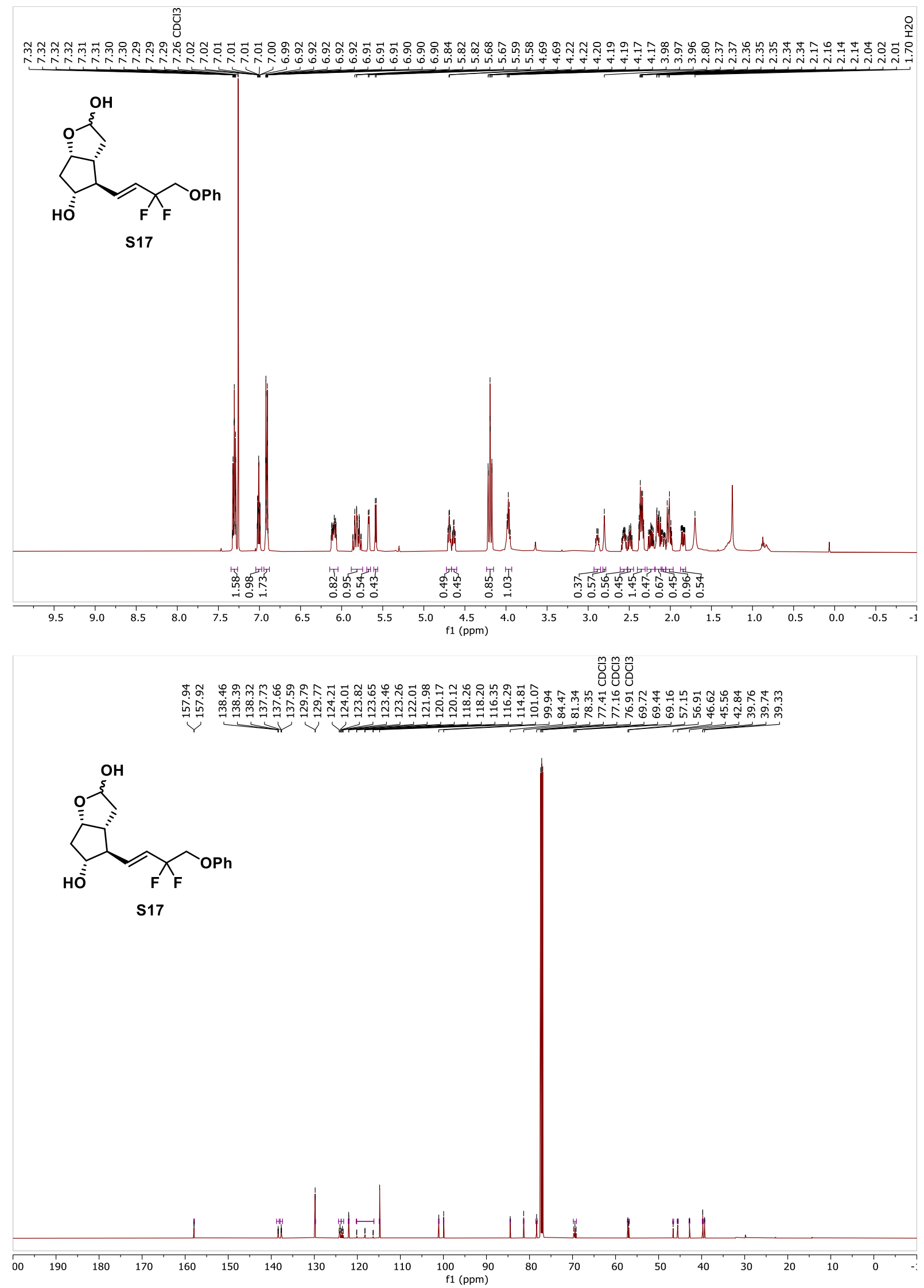

Figure S25: ${ }^{1} \mathrm{H}$ NMR (500 MHz, $\mathrm{CDCl}_{3}$, top) and ${ }^{13} \mathrm{C} \mathrm{NMR}\left(126 \mathrm{MHz}, \mathrm{CDCl}_{3}\right.$, bottom) of compound S17. 


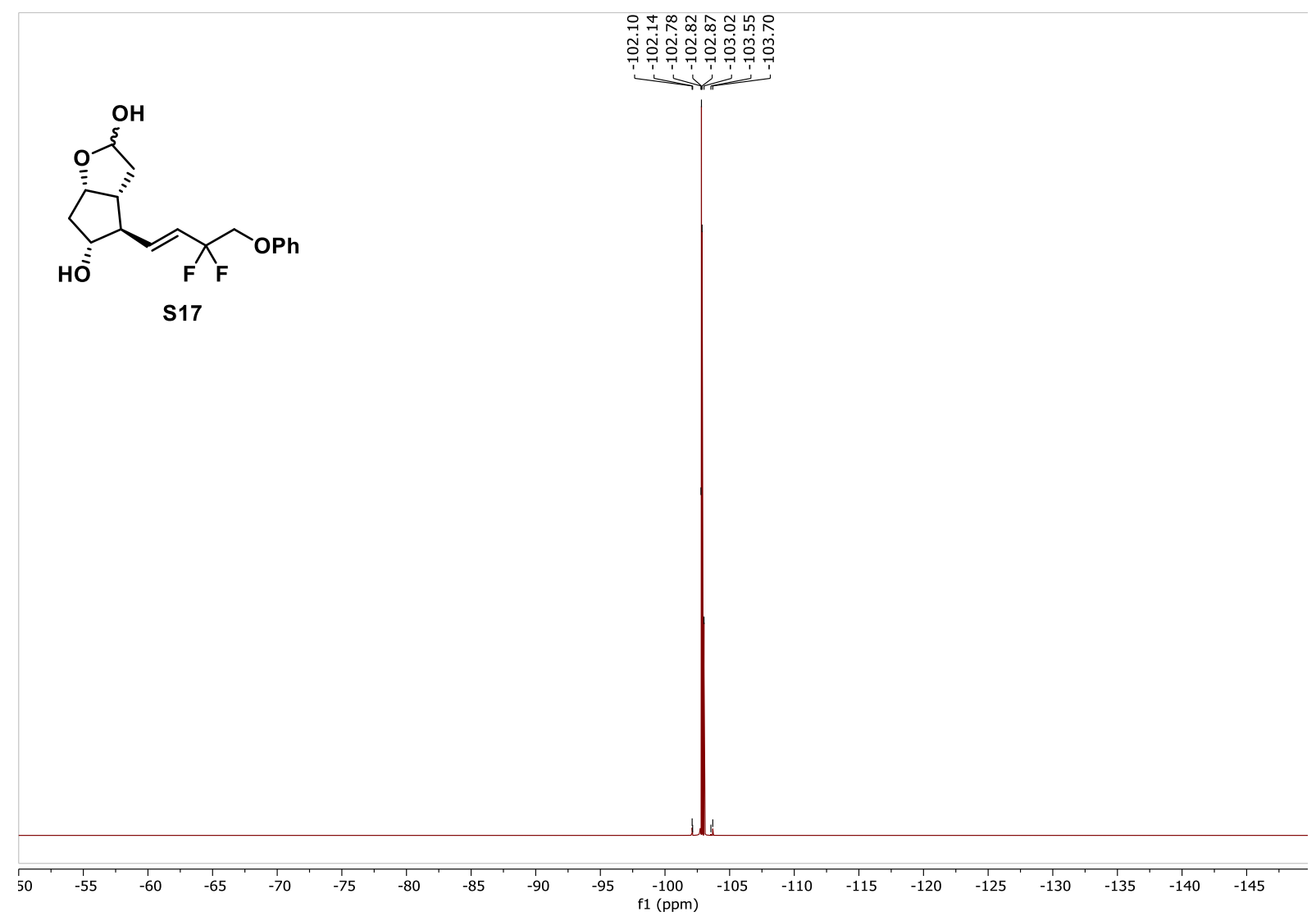

Figure S26: ${ }^{19} \mathrm{~F}$ NMR (377 MHz, $\mathrm{CDCl}_{3},{ }^{1} \mathrm{H}$ decoupled) of compound $\mathbf{S 1 7}$. 

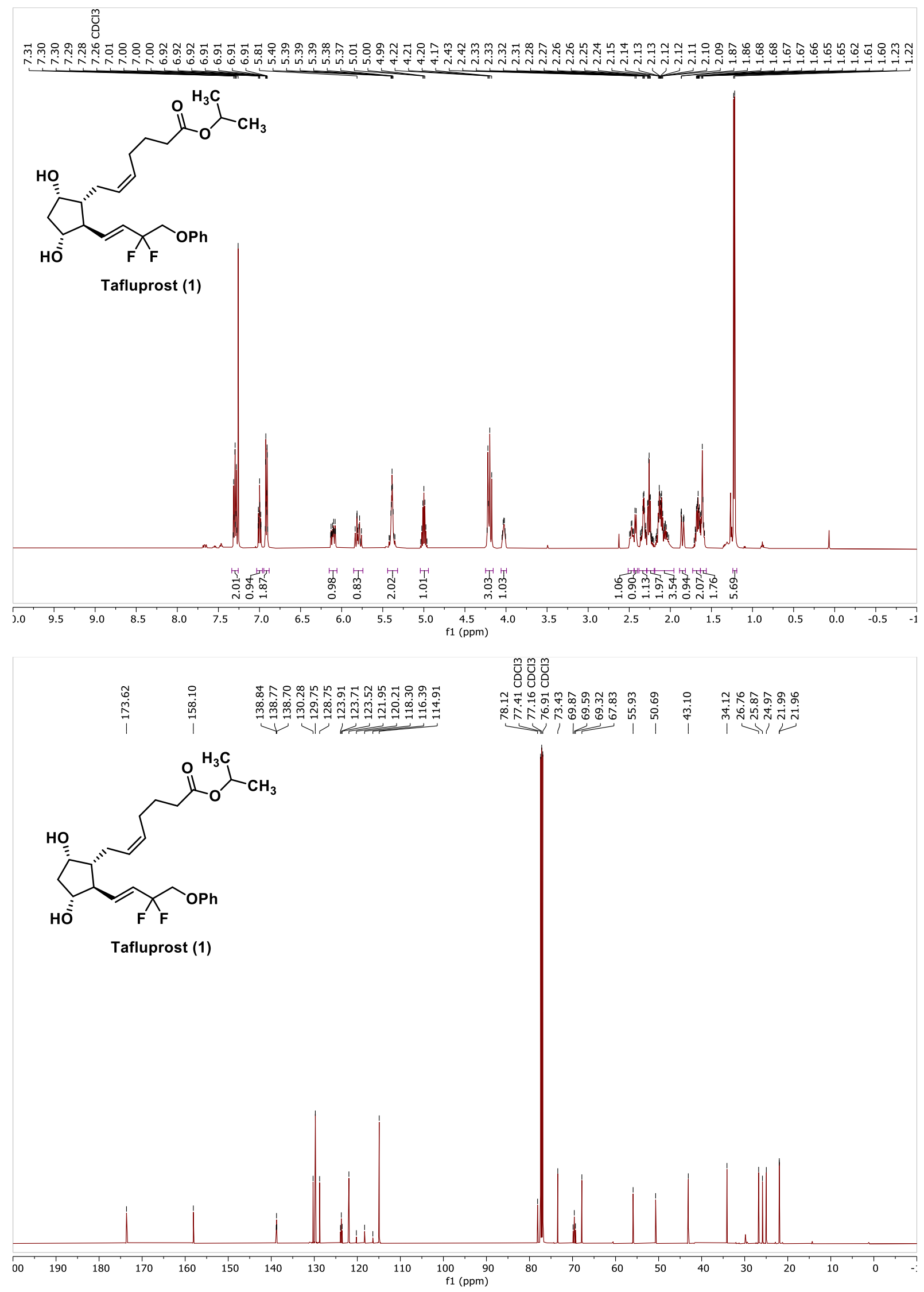

Figure S27: ${ }^{1} \mathrm{H}$ NMR $\left(500 \mathrm{MHz}, \mathrm{CDCl}_{3}\right.$, top) and ${ }^{13} \mathrm{C} \mathrm{NMR}\left(126 \mathrm{MHz}, \mathrm{CDCl}_{3}\right.$, bottom) of Tafluprost (1). 


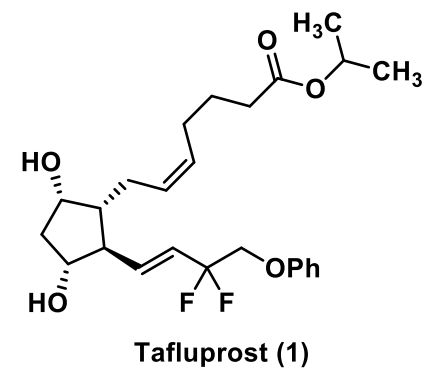

Tafluprost (1)

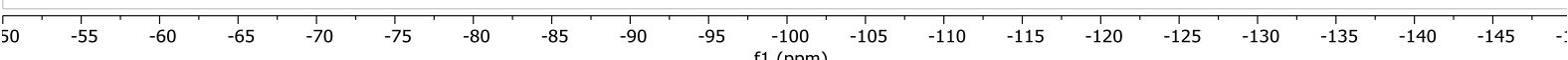

Figure S28: ${ }^{19} \mathrm{~F}$ NMR $\left(470 \mathrm{MHz}, \mathrm{CDCl}_{3},{ }^{1} \mathrm{H}\right.$ decoupled) of Tafluprost (1). 